\title{
ATOMS: ALMA Three-millimeter Observations of Massive Star-forming regions - I. Survey description and a first look at G9.62+0.19
}

Tie Liu, ${ }^{\star 1,2}$ Neal J. Evans, ${ }^{3,2}$ Kee-Tae Kim,,${ }^{2,4}$ Paul F. Goldsmith, ${ }^{5}$ Sheng-Yuan Liu, ${ }^{6}$ Qizhou Zhang, ${ }^{7}$ Ken'ichi Tatematsu, ${ }^{8}$ Ke Wang, ${ }^{9}$ Mika Juvela, ${ }^{10}$ Leonardo Bronfman, ${ }^{11}$ Maria. R. Cunningham, ${ }^{12}$ Guido Garay, ${ }^{11}$ Tomoya Hirota, ${ }^{8}$ Jeong-Eun Lee, ${ }^{13}$ Sung-Ju Kang, ${ }^{2}$ Di Li, ${ }^{14,15,27}$ Pak-Shing Li, ${ }^{16}$ Diego Mardones, ${ }^{11}$ Sheng-Li Qin, ${ }^{17}$ Isabelle Ristorcelli, ${ }^{18}$ Anandmayee Tej, ${ }^{19}$ L. Viktor Toth, ${ }^{20}$ Jing-Wen Wu, ${ }^{14}$ Yue-Fang Wu, ${ }^{21}$ Hee-weon Yi, ${ }^{13}$ Hyeong-Sik Yun, ${ }^{13}$ Hong-Li Liu, ${ }^{22}$ Ya-Ping Peng, ${ }^{23}$ Juan Li, ${ }^{24,25}$ Shang-Huo Li, ${ }^{24}$ Chang-Won Lee, ${ }^{2,4}$ Zhi-Qiang Shen, ${ }^{24,25}$ Tapas Baug, ${ }^{9}$ Jun-Zhi Wang, ${ }^{24,25}$ Yong Zhang, ${ }^{26}$ Namitha Issac, ${ }^{19}$ Feng-Yao Zhu, ${ }^{1}$ Qiu-Yi Luo, ${ }^{1}$ Archana Soam, ${ }^{28}$ Xun-Chuan Liu, ${ }^{21}$ Feng-Wei Xu, ${ }^{21}$ Yu Wang, ${ }^{21}$ Chao Zhang, ${ }^{17}$ Zhiyuan Ren, ${ }^{14}$ Chao Zhang ${ }^{14}$ Affiliations are listed at the end of the paper

Accepted XXX. Received YYY; in original form ZZZ

\begin{abstract}
The "ATOMS," standing for ALMA Three-millimeter Observations of Massive Star-forming regions, survey has observed 146 active star forming regions with ALMA Band 3, aiming to systematically investigate the spatial distribution of various dense gas tracers in a large sample of Galactic massive clumps, to study the roles of stellar feedback in star formation, and to characterize filamentary structures inside massive clumps. In this work, the observations, data analysis, and example science of the "ATOMS" survey are presented, using a case study for the G9.62+0.19 complex. Toward this source, some transitions, commonly assumed to trace dense gas, including $\mathrm{CS} J=2-1, \mathrm{HCO}^{+} J=1-0$ and $\mathrm{HCN} J=1-0$, are found to show extended gas emission in low density regions within the clump; less than $25 \%$ of their emission is from dense cores. $\mathrm{SO}, \mathrm{CH}_{3} \mathrm{OH}, \mathrm{H}^{13} \mathrm{CN}$ and $\mathrm{HC}_{3} \mathrm{~N}$ show similar morphologies in their spatial distributions and reveal well the dense cores. Widespread narrow $\mathrm{SiO}$ emission is present (over $\sim 1 \mathrm{pc}$ ), which may be caused by slow shocks from large-scale colliding flows or HII regions. Stellar feedback from an expanding HII region has greatly reshaped the natal clump, significantly changed the spatial distribution of gas, and may also account for the sequential high-mass star formation in the G9.62+0.19 complex. The ATOMS survey data can be jointly analyzed with other survey data, e.g., "MALT90", "Orion B", "EMPIRE", "ALMA_IMF", and "ALMAGAL", to deepen our understandings of "dense gas" star formation scaling relations and massive proto-cluster formation.
\end{abstract}

Key words: stars: formation - ISM: kinematics and dynamics - ISM: HII regions

\section{INTRODUCTION}

Knowledge of the physical factors that control the conversion of interstellar gas into stars is of fundamental importance for understanding the star formation process and the evolution of galaxies.
Schmidt (1959) proposed a relation between star formation rate (SFR) and gas density. Kennicutt (1998a,b) found a strong observational relation between the star formation rate (SFR) surface density $\left(\Sigma_{S F R}\right)$ and the surface density of cold gas $\left(\Sigma_{\text {gas }}\right)$, which suggests that the SFR surface density is primarily regulated by the gas mass surface density. This so-called Kennicutt-Schmidt (KS) law, is a crucial input into theoretical models of galaxy evo- 
lution. The K-S law between $\Sigma_{\mathrm{SFR}}$ and total gas surface density $\left(\Sigma_{\text {gas }}=\Sigma_{\mathrm{HI}}+\Sigma_{\mathrm{H}_{2}}\right)$ across entire galaxies and galactic nuclei, has a typical power-law index of 1.4-1.6 (Kennicutt 1998a,b; Kennicutt \& Evans 2012). Resolved studies of nearby galaxies instead show a linear relation of $\Sigma_{S F R}$ with molecular gas, implying a depletion time for gas that is constant with surface density at about 1-2 Gyr (e.g., Bigiel et al. 2008; Tacconi et al. 2020). While significant progress has been made in recent years, there are still fundamental aspects of the origin of star formation relations that remain unaddressed.

\subsection{The role of dense molecular gas in star formation}

Gao \& Solomon (2004) showed that the far-infrared luminosities $\left(L_{\mathrm{IR}}\right)$ in starburst galaxies correlated tightly with the luminosities of $\mathrm{HCN}$ line emission, i.e., the dense gas star formation law. A nearly linear relation between SFR, indicated by $L_{\mathrm{IR}}$, and dense molecular gas mass, indicated by molecular line luminosities $\left(L_{\mathrm{mol}}^{\prime}\right)$, have been revealed in a number of entire galaxies (Gao \& Solomon 2004; Greve 2014; Zhang et al. 2014; Liu et al. 2015) or spatially resolved nearby galaxies (Chen et al. 2015; Tan et al. 2018) with various dense gas tracers, e.g., $\mathrm{HCN}, \mathrm{HCO}^{+}, \mathrm{CS}$ and high-J $\mathrm{CO}$ lines. The tightness of this correlation suggests that the denser parts of molecular clouds, rather than the total clouds, provide the direct fuel for star formation.

Wu et al. $(2005,2010)$ showed that massive, dense clumps (with typical sizes of $\sim 1 \mathrm{pc}$ ) in the Milky Way followed a similar relation and suggested that the fundamental units of massive clustered star formation are such massive dense clumps. In addition, the vast majority of dense cores and young stellar objects (YSOs) in nearby clouds are found above a "threshold" surface density of about $120 \mathrm{M}_{\odot} \mathrm{pc}^{-2}$ (or $A_{\mathrm{V}} \sim 8 \mathrm{mag}$ ) (Heiderman et al. 2010; Lada et al. 2010, 2012). By comparing various models of star formation to observations of nearby clouds, Evans et al. (2014) found that the mass of dense gas above such a "threshold" surface density of $A_{\mathrm{V}} \sim 8$ mag was the best predictor of the star formation rate. Vutisalchavakul et al. (2016) found a similar result for more distant and massive clouds in the Galactic Plane, using millimeter continuum emission from the BGPS survey (Ginsburg et al. 2013) to measure the mass of dense gas. The average star formation rate per unit mass of dense gas (star formation efficiency; SFE) is remarkably constant from the scales of nearby molecular clouds to Galactic Plane clouds to nearby galaxies to distant (ultra)luminous IR galaxies (Vutisalchavakul et al. 2016). In contrast, the star formation rate per unit mass of total molecular gas shows much more dispersion (Vutisalchavakul et al. 2016).

However, the dense gas star formation relation and the nature of the gas probed by dense gas tracers such as $\mathrm{HCN}, \mathrm{HCO}^{+}$and $\mathrm{CS}$ emission is still far from being well-understood. One attractive explanation for the low star formation efficiency in molecular clouds is that most clouds are not gravitationally bound, but only relatively dense regions (clumps) within them are bound (e.g., Dobbs et al. 2011; Barnes et al. 2016). This may also explain the tight relation between far-infrared luminosities and the luminosities of dense molecular tracers in molecular clumps, i.e., dense gas star formation law (e.g., Wu et al. 2005, 2010). Liu et al. (2016) studied the dense gas star formation law for 146 Galactic clumps, most of which are gravitationally bound. They found that the slopes of the $L_{\mathrm{IR}}-L_{\mathrm{mol}}^{\prime}$ correlations vary for clumps with different dust temperatures and luminosity-to-mass ratios. Such behavior seems to be a result of different evolutionary stages of the Galactic clumps (Liu et al. 2016; Stephens et al. 2016). Recent observations of nearby clouds have also shown that some traditional high-density tracers, such as $\mathrm{HCN}(1-0)$ and $\mathrm{HCO}^{+}(1-0)$, are also easily detected in extended translucent regions at typical densities of $500 \mathrm{~cm}^{-3}$ and are poor tracers of dense structures such as filaments or cores (Kauffmann et al. 2017; Pety et al. 2017; Shimajiri et al. 2017). Most recently, Evans et al. (2020) mapped six distant ( $d \sim 3.5-10.4 \mathrm{kpc})$ clouds in $\mathrm{HCN}(1-0)$ and $\mathrm{HCO}^{+}(1-0)$ line emission with high sensitivity (rms $\sim 0.1 \mathrm{~K}$ per $0.2 \mathrm{~km} \mathrm{~s}^{-1}$ channel). They found that in all cases a substantial fraction (in most cases, the majority) of the total line luminosity arises in gas below the $A_{\mathrm{V}} \sim 8$ mag threshold. These studies have challenged the definition of dense gas associated with the observation of some commonly used tracers, e.g., $J=1-0$ of $\mathrm{HCN}$ and $\mathrm{HCO}^{+}$, but are limited in scope.

Therefore, in order to understand the origin of the dense gas star formation law, we need to address the following questions: (1) What is the spatial distribution of various molecular transitions, such as $\mathrm{CS}, \mathrm{HCN}$, and $\mathrm{HCO}^{+}$, in molecular clouds? (2) How much dense gas in the gravitationally bound clumps is actually participating in star formation or is concentrated in the smallest star formation units, i.e., dense cores that have typical sizes of $\sim 0.1 \mathrm{pc}$ ? The project introduced in this paper will systematically study how these "dense gas" tracers behave on small scales of clumps to cores, aiming for a thorough understanding of the dense gas star formation law.

For the remainder of this paper we refer to CS, HCN and $\mathrm{HCO}^{+}$without specifying the transitions. However, we emphasize that the sensitivity to density varies strongly with energy level; the transitions from levels higher than those in this study, e.g., $J=2$ for $\mathrm{CS}, J=1$ for $\mathrm{HCN}$ and $\mathrm{HCO}^{+}$, are increasingly sensitive to higher densities. Multiple transitions of a single molecular species or possibly single transitions of different species can be indicative of the characteristic densities of a region.

\subsection{The role of stellar feedback in star formation}

Stellar feedback from massive stars can strongly influence their surrounding interstellar medium and regulate star formation through proto-stellar outflows, thermal feedback, photoionizing radiation, radiation pressure, main sequence winds from hot stars or supernova explosions (e.g., Krumholz et al. 2014). The combined effects of multiple feedback mechanisms can significantly reduce star formation rates and may play a major role in determining the star formation efficiency and the stellar initial mass function in molecular clouds (e.g., Krumholz et al. 2014). Although stellar feedback usually has a negative effect on star formation, in the sense of restraining or terminating star formation, it is also possible for feedback to be positive by triggering new star formation (e.g., Elmegreen \& Lada 1977; Whitworth et al. 1994a,b; Krumholz et al. 2014; Wall et al. 2020). By studying a large sample of infrared bubbles, Thompson et al. (2012) estimated that the fraction of massive stars in the Milky Way formed by a triggering process could be between 14 and 30 percent.

With ALMA, Liu et al. (2017) recently studied sequential high-mass star formation in a proto-cluster, the G9.62+0.19 complex. The ALMA observations resolved G9.62+0.19 into a massive filament that fragments into dozens of continuum sources that are at different evolutionary stages (from high-mass starless core candidates, high-mass proto-stellar objects, hot molecular cores, to UC HII regions), as also suggested in other works (Garay et al. 1993; Hofner, Wiesemeyer, \& Henning 2001; Hofner et al. 1994, 1996; Testi et al. 2000; Liu et al. 2011; Dall'Olio et al. 2019). Liu et al. (2017) also found no evidence for widespread low-mass proto- 
stellar core population in the G9.62+0.19 complex. They suggested that the sequential high-mass star formation in this region is the outcome of the stellar feedback from evolved HII regions formed in the same natal clump. The core fragmentation may be suppressed due to feedback from young OB proto-stars by heating the cores up and injecting turbulence through outflows, leading to an increase of their Jeans masses.

In addition, thermal feedback (from outflow heating, accretion luminosity or radiative heating from OB protostars) enhanced by stellar radiation heating in conjunction with magnetic field can be important for reducing the level of fragmentation and producing massive stars (e.g., Offner et al. 2009; Krumholz et al. 2011; Myers et al. 2013). In contrast to the magnetic field, which is most effective in more diffuse regions, radiation feedback is more efficient in suppressing fragmentation in the dense, central region of proto-clusters (Myers et al. 2013). Moscadelli et al. (2018) studied the feedback of a Hyper Compact (HC) HII region on its parental molecular core in the star-forming region G24.78+0.08. They found that the ionized gas of the HC HII region is expanding into the surrounding molecular gas. The shocks produced by the fast expansion of the ionized gas significantly influence the temperature distribution throughout its parental molecular core. In addition, outflows can also heat gas at parsec scale within clumps (e.g., Wang et al. 2012).

Studies of stellar feedback in protoclusters are still rare in cases. More systematic study is needed, however, to evaluate how formed OB proto-stars in proto-clusters influence the dense gas distribution and star formation efficiency in their natal clumps. With its unprecedented sensitivity, ALMA enables such a systemic study, through observing a large sample of proto-clusters in a very reasonable integration time.

\subsection{The role of filaments in star formation}

Thermal dust emission imaging surveys with the Herschel Space Observatory have discovered ubiquitous filamentary structures in nearby clouds and distant Galactic Plane giant molecular clouds (GMCs) (e.g., André et al. 2010, 2014, 2019; Molinari et al. 2010; Arzoumanian et al. 2011; Juvela et al. 2012; Könyves et al. 2015; Wang et al. 2016; Arzoumanian et al. 2019; Schisano 2020). High resolution images from the space telescope Herschel and groundbased telescopes reveal complex internal structures inside filamentary clouds, including dense cores along or at the intersections of some of the filamentary substructures (Wang et al. 2011, 2014; André et al. 2014; Könyves et al. 2015; Zhang et al. 2015; Liu et al. 2018a,b; Kainulainen et al. 2017). Velocity coherent fiber-like substructures in filaments are also identified in nearby clouds (Hacar et al. 2013, 2016, 2018; González Lobos \& Stutz 2019) and distant infrared dark clouds (Chen et al. 2019). Observations by Herschel revealed that more than $70 \%$ of prestellar cores are embedded in larger, parsec-scale filamentary structures in nearby molecular clouds (André et al. 2014; Könyves et al. 2015). The fact that the cores reside mostly within the densest filaments with column densities exceeding $\sim 7 \times 10^{21} \mathrm{~cm}^{-2}$ strongly suggests a column density threshold for core formation (e.g., Enoch et al. 2007; André et al. 2014, and references therein).

In addition, filaments also play an important role in cluster formation (e.g., Stutz \& Gould 2016; Stutz 2018). Hub-filament systems have been revealed in high-mass star forming regions, implying a global hierarchical collapse scenario for proto-cluster formation (Liu et al. 2012, 2015b; Chen et al. 2019). Gas accreted along filaments continuously feeds the hub region, where massive stars may form (Liu et al. 2012; Kirk et al. 2013; Peretto et al. 2013; $\mathrm{Lu}$ et al. 2018; Chen et al. 2019). To date, however, only a few observational studies have been performed to trace the hypothesized accretion flows along filaments in proto-clusters.

Despite the importance of filaments in star formation, we still have too many missing links from observations to reconstruct a complete picture of star formation inside filaments. For example, do filaments in widely different environments, e.g., Galactic Plane GMCs or nearby clouds, show similar properties? Are hub-filament systems common in high-mass star-forming regions? To deepen our understandings of the roles of filaments in star formation, we need to systematically investigate a sample of filaments in both far and near clouds across the Galaxy. To separate cores from filaments in distant massive clumps, we need resolution of $<0.1 \mathrm{pc}$, which requires angular resolution of $<2^{\prime \prime}$ at distances of $<10 \mathrm{kpc}$. Only with ALMA, such a systemic high-resolution observational study is possible.

\section{ATOMS: ALMA THREE-MILLIMETER OBSERVATIONS OF 146 MASSIVE STAR-FORMING REGIONS}

To statistically investigate the star formation process in the Galaxy, we initiated the "ALMA Three-millimeter Observations of Massive Star-forming regions (ATOMS)" survey program at ALMA. The main science goals of the ATOMS project are:

- To deepen the understandings of the dense gas star formation law by studying the spatial distributions of various dense gas tracers in a large sample of Galactic clumps and evaluating how much of molecular gas is participating in star formation;

- To investigate how stellar feedback from formed OB (proto)stars influences the surrounding gas distributions and the next generation of star formation in their natal clumps;

- To resolve filaments and to study their roles in proto-cluster formation.

\subsection{The sample of ATOMS}

The ATOMS sample of 146 sources were selected from the CS $J=2-1$ survey of Bronfman et al. (1996), a complete and homogenous molecular line survey of ultra compact (UC) HII region candidates in the Galactic plane. The sample of our 146 targets is complete for proto-clusters with bright CS $J=2-1$ emission $\left(T_{\mathrm{b}}>2 \mathrm{~K}\right)$, indicative of reasonably dense gas. It is also complete for the longitude range observable by SEST as well as ALMA (Faúndez et al. 2004). Table A lists the basic parameters of this sample, including source IDs in observations (column 1), IRAS names (column 2), coordinates (columns 3-4), systemic velocities $\left(V_{\mathrm{lsr}}\right.$, column 5), distances from the sun (column 6), Galactocentric distances $\left(R_{\mathrm{GC}}\right.$, column 7$)$, effective radii $\left(R_{e f f}\right.$, column 8$)$, dust temperature ( $T_{\text {dust }}$, column 9$)$, bolometric luminosity ( $L_{\text {bol }}$, column 10 ) and clump masses ( $M_{\text {clump }}$, column 11). There are 122 clumps with ATLASGAL and HiGAL counterparts, which have been analyzed in detail by Urquhart et al. (2018). The basic parameters for these 122 clumps are adopted from Urquhart et al. (2018). The basic parameters for the remaining clumps are adopted from Faúndez et al. (2004).

Figure 1 shows the Galactic distribution of the ATOMS targets on the Planck $857 \mathrm{GHz}$ emission map. Figure 2 presents the 


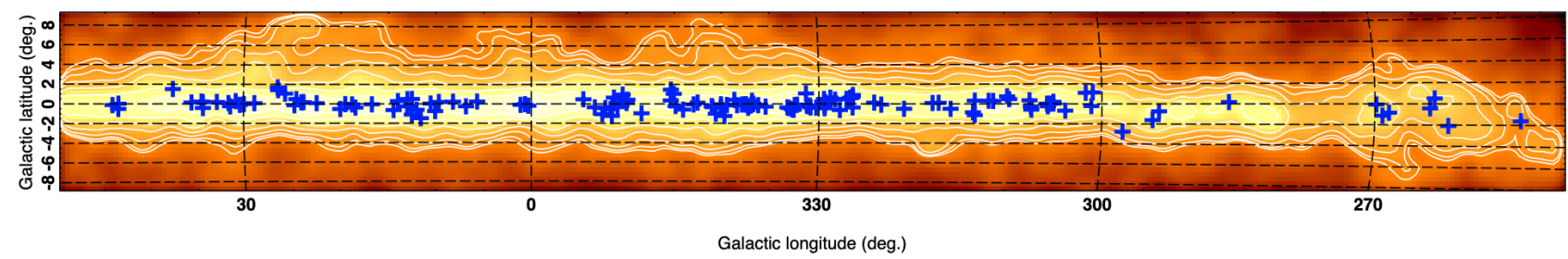

Figure 1. Galactic distribution of the ATOMS targets ("+"). The background image and contours show Planck $857 \mathrm{GHz}$ emission. The contours are from 34.6 to $874.5 \mathrm{MJy} / \mathrm{sr}$ with 10 steps on a logarithmic scale.

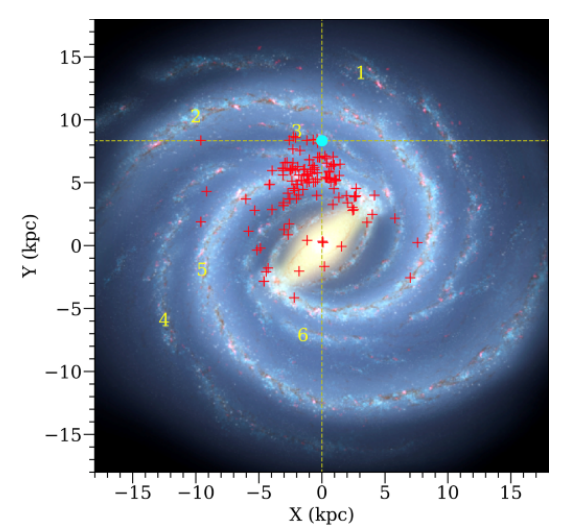

Figure 2. Spatial distribution of the ATOMS targets ("+") projected onto a top-down schematic view of the Milky Way (artist's concept, R. Hurt: NASA/JPLCaltech/ SSC). The spiral arms are indicated using numbers from 1 to 6 , referring to the Outer, Perseus, Local, Carina-Sagittarius, Scutum-Centaurus, and Norma arms. (Codes used for this plot is from Yuan et al. (2017)).

spatial distribution of the ATOMS targets on a top-down schematic view of the Milky Way. From them, we can see that most (139) of the ATOMS targets are located in the first and fourth Galactic Quadrants of the inner Galactic Plane $\left(|b|<2^{\circ}\right)$. The distances of the sample clouds range from $0.4 \mathrm{kpc}$ to $13.0 \mathrm{kpc}$ with a mean value of $4.5 \mathrm{kpc}$. About $67 \%$ of the sources are located closer than $\sim 5 \mathrm{kpc}$ (see Figure 2). We include 27 distant $(d>7 \mathrm{kpc}$ ) sources that are either close to the Galactic center or mini-starbursts (like W49N/I19078+0901), representing extreme environments for star formation. The sources have Galactocentric distances ranging from $0.5 \mathrm{kpc}$ to $12.7 \mathrm{kpc}$, and are mostly located on spiral arms (see Figure 2).

Figure 3 a presents the distribution of the clump masses of the sample. The clump masses range from 5.6 to $2.5 \times 10^{5} M_{\odot}$, with a median value of $1.4 \times 10^{3} \mathrm{M}_{\odot}$. The distribution of the bolometric luminosity of targets is shown in Figure $3 \mathrm{~b}$. The bolometric luminosity ranges from 16 to $8.1 \times 10^{6} \mathrm{~L}_{\odot}$, with a median value of $5.7 \times 10^{4} \mathrm{~L} \odot$. All the targets in the sample have bolometric luminosity larger than $1 \times 10^{3} \mathrm{~L}_{\odot}$ except for I08076-3556 and I1 15906452. I08076-3556, also known as BHR 12 or CG 30, is a brightrimmed cometary globule located in the Gum Nebula region at a distance of $\sim 400 \mathrm{pc}$, in which a low-mass binary system is forming (Chen et al. 2008). I11590-6452 (also known as BHR 71) is also a bright-rimmed cometary globule that is forming a wide binary system (Tobin et al. 2019).

The radii of the sample range from 0.06 to $4.26 \mathrm{pc}$, with a median value of $0.86 \mathrm{pc}$, suggesting that the sample contains both nearby dense cores $\left(R_{\text {eff }} \sim 0.1 \mathrm{pc}\right)$ and distant clouds ( $R_{\text {eff }}$ of several pc). The dust temperature ranges from 18 to $46 \mathrm{~K}$, with a median value of $29 \mathrm{~K}$. Liu et al. (2016) observed this sample in HCN (4-3) and CS (7-6) lines. The line widths of $\mathrm{HCN}$ (4-3) range from 1.5 to $21 \mathrm{~km} \mathrm{~s}^{-1}$, with a median value of $6.4 \mathrm{~km} \mathrm{~s}^{-1}$. The line widths of CS (7-6) range from 1.6 to $23 \mathrm{~km} \mathrm{~s}^{-1}$, with a median value of 4.5 $\mathrm{km} \mathrm{s}^{-1}$. Most of the targets tend to be gravitationally bound with an average virial parameter of $\sim 0.8$ (Liu et al. 2016).

In a word, this is a very diverse sample that is suitable for statistical studies of the star formation process with different conditions, e.g., different density, temperature, turbulence level and luminosity of dominating OB protostars, in widely different Galactic environments covering a large range of Galactocentric distances.

Figure $3 \mathrm{c}$ shows the mass-radius diagram for the ATOMS sample. All the targets are above the mass surface density thresholds for "efficient star formation" given in Lada et al. (2010) and Heiderman et al. (2010), implying that they are active star-forming regions. We notice that only three of the ATOMS targets are above the surface density threshold of $1 \mathrm{~g} \mathrm{~cm}^{-2}$, which is a stringent threshold for high-mass star formation given by Krumholz \& McKee (2008). However, there are 134 clumps having mass surface density above the threshold for high-mass star formation regions, $M>870 M_{\odot}(r / p c)^{1.33}$, defined by Kauffmann \& Pillai (2010). Based on studies of ATLASGAL clumps, Urquhart et al. (2014) also suggested a less stringent empirical threshold of $0.05 \mathrm{~g} \mathrm{~cm}^{-2}$ for high-mass star formation. There are 132 clumps fulfilling the thresholds given by Kauffmann \& Pillai (2010) and Urquhart et al. (2014), suggesting that more than $90 \%$ of the ATOMS targets have the ability to form high-mass stars. Figure $3 \mathrm{~d}$ shows the luminositymass diagram for the ATOMS sample. About 143 ATOMS targets are above the evolution tracks for stars with final masses larger than $8 \mathrm{M}_{\odot}$, indicating that the majority of the clouds are forming highmass stars. The majority of the clumps are in the accelerated accretion phase (Molinari et al. 2008).

In the below, we describe the observations, data analysis, and illustrate the science goals of the ATOMS survey, using a case study for the G9.62+0.19 complex (known as I18032-2032 in Table A), which is an active high-mass star forming region located at a distance of $5.2_{-0.6}^{+0.6} \mathrm{kpc}$ (from trigonometric parallax of $12 \mathrm{GHz}$ methanol masers; Sanna et al. 2009). The G9.62+0.19 clump is unstable against gravitational collapse even if thermal, turbulent, and magnetic field support are taken into account together (Liu et al. 2018c). We should note that throughout this paper the analysis of G9.62+0.19 and comparison with previous works, e.g., Liu et al. (2017), is only applicable to this particular source. The ATOMS will allow to extend the analysis to a much larger sample of sources.

\subsection{ALMA Observations}

The ALMA observations of the ATOMS survey were conducted from late September to mid November 2019 with both the Atacama Compact $7 \mathrm{~m}$ Array (ACA; Morita Array) and the 12-m 
Table 1. Observation logs of the ATOMS survey.

\begin{tabular}{|c|c|c|c|c|c|c|}
\hline Source IDs & Obs date & $\underset{(\mathrm{m})}{\operatorname{Min} \mathrm{BL}}$ & $\underset{(\mathrm{m})}{\operatorname{Max} B L}$ & $\begin{array}{l}\text { Angular resolution } \\
\text { (") }\end{array}$ & $\begin{array}{l}\text { Maximum recovering scale } \\
\left({ }^{\prime \prime}\right)\end{array}$ & $\begin{array}{c}\text { rms per } 0.122 \mathrm{MHz} \text { channel } \\
\left(\mathrm{mJy}^{-1} \text { beam }^{-1}\right)\end{array}$ \\
\hline \multicolumn{7}{|c|}{ ACA Observations } \\
\hline 1 & 2019-10-14 & 8.9 & 48.0 & 13.3 & 76.2 & 49 \\
\hline $2-7$ & 2019-10-14 & 8.9 & 48.0 & 13.3 & 76.2 & 63 \\
\hline 8 & 2019-10-19 & 8.9 & 48.0 & 13.3 & 72.9 & 46 \\
\hline $9-11$ & 2019-11-08 & 8.9 & 48.0 & 13.3 & 76.2 & 70 \\
\hline $12-23$ & $2019-11-15$ & 8.9 & 48.0 & 13.3 & 76.2 & 70 \\
\hline $24-30$ & 2019-11-13,2019-11-17 & 8.9 & 44.7 & 13.5 & 76.2 & 70 \\
\hline $31-36$ & $2019-11-12$ & 9.6 & 48.0 & 13.1 & 53.8 & 61 \\
\hline $37-62$ & 2019-11-17,2019-11-18 & 8.9 & 48.0 & 13.3 & 76.2 & 61 \\
\hline $63-81$ & 2019-11-12 & 8.9 & 48.0 & 13.1 & 76.2 & 93 \\
\hline $82-88$ & 2019-10-31 & 8.9 & 45.0 & 13.8 & 76.2 & 58 \\
\hline $89-101$ & 2019-11-19 & 8.9 & 48.0 & 13.3 & 76.2 & 83 \\
\hline $102-104$ & 2019-09-30 & 9.6 & 48.0 & 13.1 & 53.8 & 51 \\
\hline $105-110$ & 2019-10-01,2019-10-13 & 9.1 & 48.0 & 13.1 & 65.4 & 48 \\
\hline $111-123$ & 2019-11-02, 2019-11-03 & 8.9 & 44.7 & 13.5 & 76.2 & 80 \\
\hline $124-131$ & 2019-10-31,2019-11-01 & 8.9 & 48.0 & 13.3 & 76.2 & 51 \\
\hline $132-143$ & 2019-11-01,2019-11-04 & 8.9 & 48.0 & 13.3 & 76.2 & 64 \\
\hline $144-146$ & 2019-09-30 & 9.6 & 48.0 & 13.1 & 53.8 & 67 \\
\hline \multicolumn{7}{|c|}{ ALMA 12-m Observations } \\
\hline 1 & 2019-10-19 & 15.1 & 783.5 & 1.2 & 14.5 & 3.7 \\
\hline $2-7$ & 2019-10-19 & 15.1 & 783.5 & 1.2 & 14.5 & 4.5 \\
\hline 8 & 2019-11-02 & 15.1 & 500.2 & 1.7 & 19.0 & 6.2 \\
\hline $9-11$ & 2019-11-11 & 15.1 & 500.2 & 1.8 & 20.3 & 8.1 \\
\hline $12-23$ & $2019-11-13$ & 15.3 & 500.2 & 1.7 & 19.3 & 9.3 \\
\hline $24-30$ & $2019-11-12$ & 15.1 & 500.2 & 1.8 & 19.5 & 9.6 \\
\hline $31-36$ & 2019-11-04 & 15.1 & 500.2 & 1.8 & 20.3 & 5.9 \\
\hline $37-62$ & 2019-11-04 & 15.1 & 500.2 & 1.7 & 19.9 & 9.0 \\
\hline $63-81$ & 2019-11-03 & 15.1 & 500.2 & 1.7 & 20.1 & 6.4 \\
\hline $82-88$ & 2019-11-03 & 15.1 & 500.2 & 1.9 & 21.1 & 9.2 \\
\hline 89-101 & 2019-11-03 & 15.1 & 500.2 & 1.7 & 20.1 & 8.4 \\
\hline $102-104$ & $2019-10-28$ & 15.1 & 696.8 & 1.4 & 18.1 & 6.6 \\
\hline $105-110$ & 2019-10-31 & 15.1 & 696.8 & 1.5 & 19.9 & 8.1 \\
\hline $111-123$ & 2019-11-01 & 15.1 & 500.2 & 1.7 & 18.3 & 8.5 \\
\hline $124-131$ & $2019-10-31$ & 15.1 & 696.8 & 1.5 & 19.9 & 7.4 \\
\hline $132-143$ & 2019-10-31 & 15.1 & 696.8 & 1.5 & 19.9 & 5.4 \\
\hline $144-146$ & 2019-10-31 & 15.1 & 696.8 & 1.5 & 19.9 & 7.9 \\
\hline
\end{tabular}

Table 2. Main targeted molecular lines in the ATOMS survey.

\begin{tabular}{|c|c|c|c|c|c|c|c|c|c|}
\hline spw & $\begin{array}{l}\text { bandwidth } \\
(\mathrm{MHz})\end{array}$ & $\begin{array}{c}\delta V \\
\left(\mathrm{~km} \mathrm{~s}^{-1}\right)\end{array}$ & Species & Transition & $\begin{array}{c}\text { Rest frequency } \\
(\mathrm{GHz})\end{array}$ & $\begin{array}{c}\mathrm{E}_{u} / \mathrm{k} \\
(\mathrm{K})\end{array}$ & $\begin{array}{c}\mathrm{n}_{\text {crit }}^{a}(100 \mathrm{~K}) \\
\left(\mathrm{cm}^{-3}\right)\end{array}$ & $\begin{array}{c}\mathrm{n}_{e f f}^{a}(100 \mathrm{~K}) \\
\left(\mathrm{cm}^{-3}\right)\end{array}$ & Note \\
\hline spw1 & 58.59 & 0.424 & $\mathrm{H}^{13} \mathrm{CN}$ & $1-0$ & 86.339918 & 4.14 & $9.7 \times 10^{4}$ & $6.5 \times 10^{4}$ & high-density tracer \\
\hline spw2 & 58.59 & 0.422 & $\mathrm{H}^{13} \mathrm{CO}^{+}$ & $1-0$ & 86.754288 & 4.16 & $2.0 \times 10^{4}$ & \multirow[t]{3}{*}{$1.1 \times 10^{4}$} & high-density and ionization tracer \\
\hline spw3 & 58.59 & 0.419 & $\mathrm{CCH}$ & $1-0$ & 87.316898 & 4.19 & $1.9 \times 10^{5}$ & & tracer of photodissociation regions \\
\hline spw4 & 58.59 & 0.421 & $\mathrm{SiO}$ & $2-1$ & 86.84696 & 6.25 & $1.7 \times 10^{5}$ & & shock/outflow tracer \\
\hline spw5 & 58.59 & 0.206 & $\mathrm{HCN}$ & $1-0$ & 88.631847 & 4.25 & $1.1 \times 10^{5}$ & $1.7 \times 10^{3}$ & high-density/infall/outflow tracer \\
\hline spw6 & 58.59 & 0.205 & $\mathrm{HCO}^{+}$ & $1-0$ & 89.188526 & 4.28 & $2.3 \times 10^{4}$ & $2.6 \times 10^{2}$ & high-density/infall/outflow tracer \\
\hline \multirow[t]{4}{*}{ spw7 } & 1875.00 & 2.973 & $\mathrm{CH}_{3} \mathrm{OH}$ & $2(1,1)-1(1,0) \mathrm{A}$ & 97.582798 & 21.56 & $4.8 \times 10^{4}$ & \multirow{4}{*}{$4.7 \times 10^{3}$} & high-density/hot-cores/shock tracer \\
\hline & & & CS & $2-1$ & 97.980953 & 7.05 & $5.5 \times 10^{4}$ & & high-density/infall/outflow tracer \\
\hline & & & SO & $3(2)-2(1)$ & 99.29987 & 9.23 & $3.0 \times 10^{5}$ & & high-density/shock/outflow tracer \\
\hline & & & $\mathrm{H}_{\alpha}$ & $\mathrm{H}_{40 \alpha}$ & 99.022952 & & & & tracer of ionized gas/HII regions \\
\hline spw8 & 1875.00 & 2.914 & $\mathrm{HC}_{3} \mathrm{~N}$ & $11-10$ & 100.07639 & 28.82 & $9.2 \times 10^{4}$ & $1.1 \times 10^{4}$ & high-density/hot-cores tracer \\
\hline
\end{tabular}

\footnotetext{
$a$ The critical density and effective excitation density are from Shirley (2015).
} 

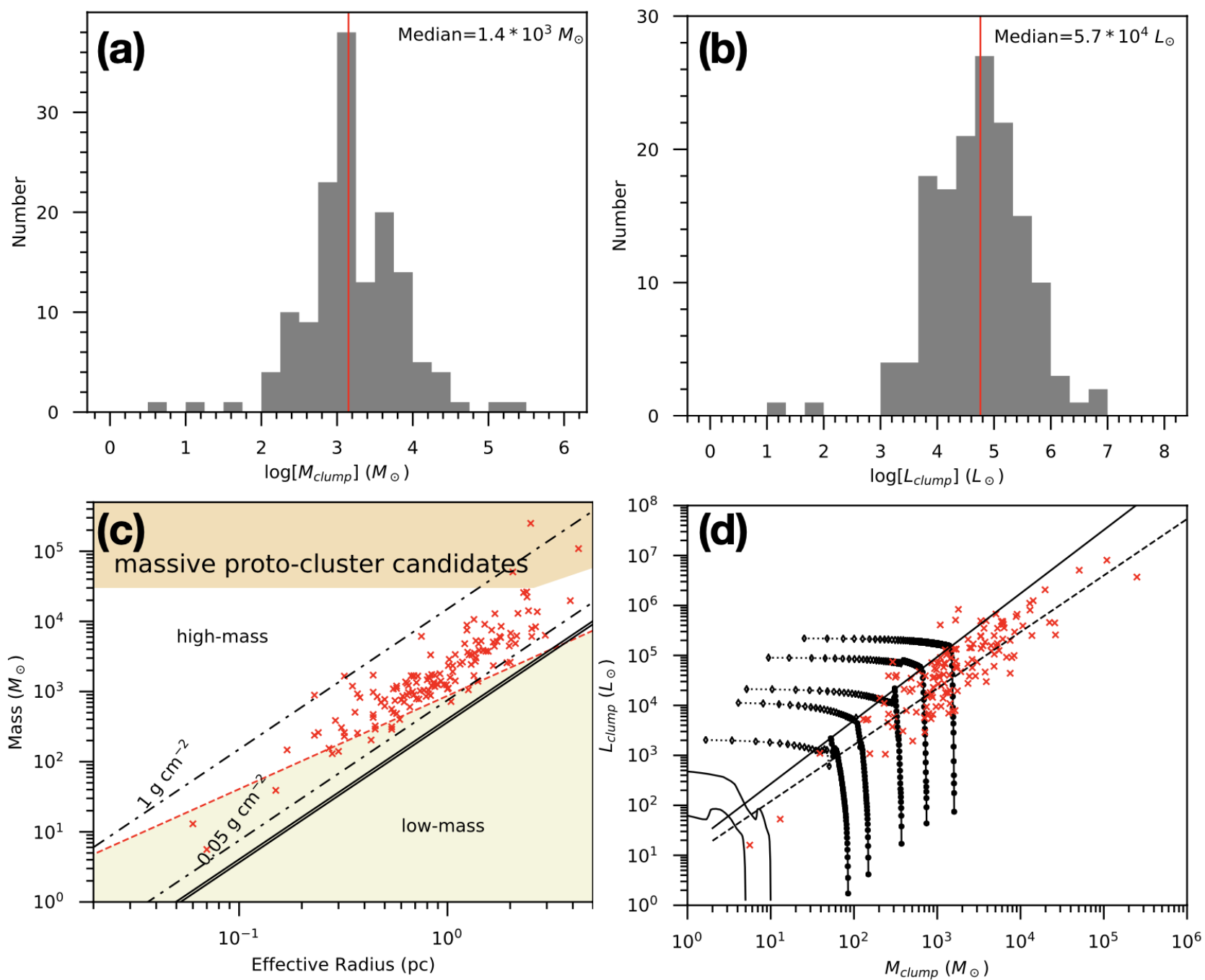

Figure 3. (a) Distribution of clump masses of the ATOMS targets; the red line shows the median value; (b) Distribution of clump luminosity; (c) $M_{\text {clump }}$ as a function of effective radii for ATOMS targets (red crosses). The unshaded area (above the red dashed line) delimits the region of high-mass star formation regions above the threshold $M>870 M_{\odot}(r / p c)^{1.33}$ defined by Kauffmann \& Pillai (2010). The black solid lines show the mass surface density thresholds for "efficient star formation" of $116 M_{\odot} \mathrm{pc}^{-2}\left(\sim 0.024 \mathrm{~g} \mathrm{~cm}^{-2}\right)$ from Lada et al. (2010) and $129 M_{\odot} \mathrm{pc}^{-2}\left(\sim 0.027 \mathrm{~g} \mathrm{~cm}^{-2}\right)$ from Heiderman et al. $(2010)$. The upper and lower dot-dashed lines show two mass surface density thresholds of 0.05 and $1 \mathrm{~g} \mathrm{~cm}^{-2}$ for high-mass star formation, which are given by Urquhart et al. (2014) and Krumholz \& McKee (2008), respectively. The upper shaded region indicates the parameter space for massive protoclusters, defined in Bressert et al. (2012). (d) Luminosity-mass diagram for ATOMS targets (red "+"). Evolutionary tracks for stars with final masses of 2.0, 4.0, 6.5, 8.0, 13.5, 18.0, and $35.0 M_{\odot}$ are from Saraceno et al. (1996, solid tracks) and Molinari et al. (2008, tracks with symbols). The solid and dashed lines are the best log-log fit for Class I and Class 0 sources, respectively, extrapolated in the high-mass regime by Molinari et al. (2008). (Codes used for panels c, d are from Yuan et al. (2017)).

array (C43-2 or C43-3 configurations) with band 3 (Project ID: 2019.1.00685.S; PI: Tie Liu). The observations including Source IDs, observation dates, minimum baselines, maximum baselines, angular resolution, Maximum recovering scale (MRS) and rms level per $0.122 \mathrm{MHz}$ channel in the ATOMS survey are summarized in Table 1. The ACA observations of G9.62+0.19 (I180322032, number 108 in Table 1) were conducted on the 1st and 13th October, 2019, with two executions. The 12-m array observations of G9.62+0.19 were conducted on the 31st October, 2019.

We conducted single-pointing observations with ALMA. The typical $12-\mathrm{m}$ array time on source is $\sim 3$ minutes, while typical ACA observing time per source is $\sim 8$ minutes. The angular resolution and maximum recovered angular scale for the ACA observations are $\sim 13.1^{\prime \prime}-13.8^{\prime \prime}$ and $\sim 53.8^{\prime \prime}-76.2^{\prime \prime}$, respectively. The angular resolution and maximum recovering angular scale for the $12-\mathrm{m}$ array observations are $\sim 1.2^{\prime \prime}-1.9^{\prime \prime}$ and $\sim 14.5^{\prime \prime}-20.3^{\prime \prime}$, respectively. These ACA and 12-m array observations are sensitive to angular scales smaller than $\sim 60^{\prime \prime}$ and $\sim 20^{\prime \prime}$, respectively. The achieved angular resolutions of the 12-m array observations are usually better than $2^{\prime \prime}$, enabling us to resolve dense cores $(\sim 0.1 \mathrm{pc}$ in size; or $2^{\prime \prime}$ at $10 \mathrm{kpc}$ distance) in the most distant sources. The obtained sensitivity in 12-m array observations ranges from 3.7 to $9.6 \mathrm{mJy}$ beam per $0.122 \mathrm{MHz}$ channel.

The observations employed the Band 3 receivers in dualpolarization mode. Eight spectral windows (SPWs) were configured to cover 11 commonly-used lines including the dense gas tracers (e.g., $J=1-0$ of $\mathrm{HCO}^{+}, \mathrm{HCN}$ and their isotopes), hot core tracers (e.g., $\mathrm{CH}_{3} \mathrm{OH}, \mathrm{HC}_{3} \mathrm{~N}$ ), shock tracers (e.g., $\mathrm{SiO}, \mathrm{SO}$ ) and ion- 
ized gas tracers $\left(\mathrm{H}_{40 \alpha}\right)$, as listed in Table 2. The second and third columns of Table 2 list the total bandwidth and spectral resolution of each spectral window (SPW, column 1). The specie names, transitions, rest frequencies and upper energies are listed in columns 4-7. The critical density $\left(\mathrm{n}_{\text {crit }}\right)$, as the density for which the net radiative decay rate from $\mathrm{j}$ to $\mathrm{k}$ equals the rate of collisional depopulation out of the upper level $\mathrm{j}$ for a multi-level system, of these transitions at $100 \mathrm{~K}$ are shown in the 8th column. In the 9th column, we also listed the effective excitation density $\left(\mathrm{n}_{\text {eff }}\right)$ at 100 $\mathrm{K}$, the density which results in a molecular line with an integrated intensity of $1 \mathrm{~K} \mathrm{~km} \mathrm{~s}^{-1}$ (Evans 1999; Shirley 2015).

Two wide SPWs, each one having $1875 \mathrm{MHz}$ bandwidth and $\sim 3 \mathrm{~km} \mathrm{~s}^{-1}$ spectral resolution are used for continuum emission and line scan survey. The SPWs (1-6) at the lower band have sufficiently high spectral resolution $\left(\sim 0.2-0.4 \mathrm{~km} \mathrm{~s}^{-1}\right)$ to resolve line profiles in high-mass star forming regions (typical line width of several $\mathrm{km} \mathrm{s}^{-1}$ ) for studying kinematics.

Calibration and imaging were carried out using the CASA software package version 5.6 (McMullin et al. 2007). The ACA data and $12-\mathrm{m}$ array data were calibrated and imaged separately.

The ACA data and 12-m array data can be combined together to recover very extended emission that is missed in 12-m array observations. In Figure 4, we simply combine the 12-m array data and ACA data of G9.62+0.19 in Fourier space with the CASA task "feather" for $\mathrm{HCO}^{+}$and $\mathrm{H}^{13} \mathrm{CO}^{+}$. Panels a and $\mathrm{c}$ of Figure 4 present the spectra of $\mathrm{HCO}^{+}$and $\mathrm{H}^{13} \mathrm{CO}^{+}$, respectively. We found that $12-\mathrm{m}$ array data alone has recovered more than $90 \%$ of the flux in both $\mathrm{H}^{13} \mathrm{CO}^{+}$and $\mathrm{HCO}^{+}$emission. Panels $\mathrm{b}$ and $\mathrm{d}$ of Figure 4 show their integrated intensity maps from 1.5 to $6.5 \mathrm{~km} \mathrm{~s}^{-1}$. The morphology of integrated intensity maps constructed with $12-\mathrm{m}$ array data alone is nearly identical to that of the maps constructed with combined data (see Figure $4 \mathrm{~b}$ and $4 \mathrm{~d}$ ). We will more properly combine the ACA data and 12-m array data in future studies and discuss the data combination issue in a forthcoming paper (Wang et al. 2020, in preparation). The combined data, however, also suffer a non trivial missing flux problem without zero-spacing information from Total-Power (TP) observations. However, this is also advantageous for studying dense structures inside these "ATOMS" clumps. This filters out the large scale emission, making it easier the identification of compact cores or dense filamentary structures (see sections 3.1 and 3.2.6). We should note that dense cores are usually very compact (see Table 3 for example) and have angular sizes much smaller than the maximum recovering angular scale $\left(\sim 20^{\prime \prime}\right)$ in the 12-m array observations, indicating that the missing flux will not be a problem in deriving physical parameters (like fluxes, densities and masses) for dense cores using the $3 \mathrm{~mm}$ continuum emission.

In this work, we mainly focus on the spatial distributions of various gas tracers. The 12-m array data alone are good enough for this purpose. Hence, in this paper, the ACA data and 12-m array data were not combined for the case study of G9.62+0.19.

We construct continuum visibility data using the line-free spectral channels for G9.62+0.19. The continuum image of G9.62+0.19 from 12-m array data reaches a $1 \sigma$ rms noise of 0.2 mJy in a synthesized beam of $1.56^{\prime \prime} \times 1.38^{\prime \prime}\left(\right.$ P.A. $\left.=87.62^{\circ}\right)$. The synthesized beams for molecular lines of G9.62+0.19 are slightly different from those for continuum. The typical synthesized beam is $1.9^{\prime \prime} \times 1.7^{\prime \prime}$ for lines at lower band (SPWs 1-6), and is $1.6^{\prime \prime} \times 1.4^{\prime \prime}$ for lines at upper band (SPWs 7-8). The $1 \sigma$ rms noise is about 8 mJy beam ${ }^{-1}$ at SPWs $1-4$ per $0.122 \mathrm{MHz}$ channel. SPWs 5-6 have the best spectral resolution of $0.06 \mathrm{MHz}$ and their $1 \sigma \mathrm{rms}$ noise is about $10 \mathrm{mJy}$ beam $^{-1}$ per channel. The $1 \sigma \mathrm{rms}$ noise at the two wide SPWs is about $3 \mathrm{mJy}^{\text {beam }}{ }^{-1}$ per channel. The continuum image constructed from ACA data reaches a $1 \sigma$ rms noise of $4 \mathrm{mJy}$ in a synthesized beam of $17.23^{\prime \prime} \times 9.46^{\prime \prime}\left(\right.$ P.A. $\left.=-82.87^{\circ}\right)$. The $1 \sigma$ rms noise for the ACA observations are $\sim 40 \mathrm{mJy}^{\text {beam }}{ }^{-1}, \sim 80$ mJy beam ${ }^{-1}$, and $\sim 15 \mathrm{mJy}$ beam $^{-1}$ per channel for lines in SPW 1-4, SPWs 5-6, and SPWs 7-8, respectively. The angular resolution and sensitivity achieved in observations of G9.62+0.19 are comparable to the median values of the whole sample.

\section{SCIENCE GOALS AND A FIRST LOOK AT G9.62+0.19}

In this section, we present the case study of the proto-cluster complex, G9.62+0.19, and introduce the exemplar sciences cases that can be addressed with the ATOMS survey.

\subsection{Filaments and dense cores}

The $3 \mathrm{~mm}$ continuum emission will be used to study how the massive clumps fragment into filaments and individual dense cores that will form new stars.

Figure 5 shows the $3 \mathrm{~mm}$ continuum emission of G9.62+0.19. The $3 \mathrm{~mm}$ continuum emission from ACA observations is shown as contours in Figure 5a and reveals only one single clump. The $3 \mathrm{~mm}$ continuum emission from high-resolution 12-m array data is shown as gray image in Figure $5 \mathrm{a}$ and contours in $5 \mathrm{~b}$, which reveals the expanding cometary-like HII region, "B", to the west, and a massive fragmented filament to the east.

In the ATOMS survey, we apply the FellWalker algorithm in the Starlink CUPID package (Berry et al. 2007, 2015), for source extraction. The core of the FellWalker algorithm is a gradienttracing scheme consisting of following many different paths of steepest ascent in order to reach a significant summit, each of which is associated with a clump (Berry et al. 2007). FellWalker is less dependent on specific parameter settings than CLUMPFIND (Berry et al. 2007), and has been widely used in continuum surveys (e.g., Eden et al. 2017, 2019; Liu et al. 2018a). Source extraction was conducted in regions above a threshold of $3 \sigma$. The minimum number of contiguous pixels (pixel size $=0.27^{\prime \prime}$ ) was set at 12 , equaling to those contained in half of the synthesized beam, which was used to define the smallest structures, i.e., "cores" (see Sanhueza et al. 2019). We also tested FellWalker with larger minimum number (16 and 25) of contiguous pixels and found that the results were not changed. The detected sources were fitted with a 2D Gaussian profile and de-convolved with the beam size. With FellWalker, we identified six main components in the $3 \mathrm{~mm}$ continuum emission of G9.62+0.19, as shown in Figure 5a.Their de-convolved sizes, peak flux density and total flux density of the $3 \mathrm{~mm}$ continuum emission are listed in columns 2-4 of Table 3. Another component, MM7/G, is not resolved in $3 \mathrm{~mm}$ continuum, but is resolved in other hot core tracers like $\mathrm{HC}_{3} \mathrm{~N}$ (11-10). MM7/G is also marked on Figure $5 \mathrm{~b}$ and listed in Table 3 .

At $3 \mathrm{~mm}$, both thermal dust emission and free-free emission from ionized gas could contribute to the continuum emission. However, since not all the ATOMS targets have been observed at centimeter wavelengths, we cannot easily compile centimeter continuum emission data to help removing any free-free contribution to the $3 \mathrm{~mm}$ continuum emission. Instead, we use the $\mathrm{H}_{40 \alpha}$ radio recombination line (RRL) to estimate the contribution of free-free emission in continuum by deriving the line-to-continuum intensity ratio (Condon \& Ransom 2016; Liu et al. 2019). The integrated intensity map of $\mathrm{H}_{40 \alpha}$ is shown as color image in Figure $5 \mathrm{~b} . \mathrm{H}_{40 \alpha}$ 

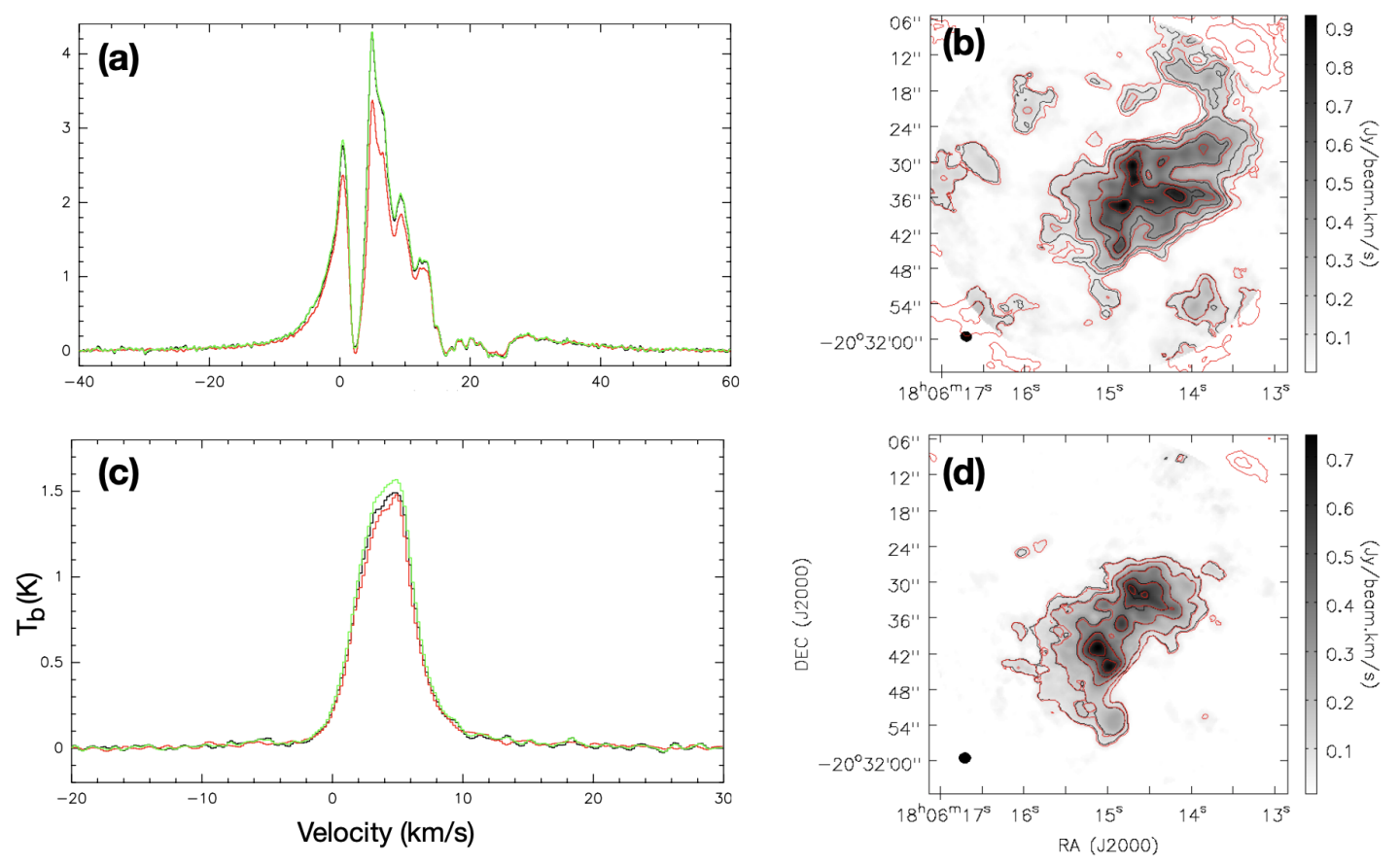

Figure 4. (a) $\mathrm{HCO}^{+}$spectra in G9.62+0.19 averaged over a 30" region at the central position (R.A.(J2000)=18:06:14.99, decl.(J2000)=-20:31:35.4). The spectra from ACA data, 12-m array data and combined 12-m and ACA data are shown in black, red and green, respectively. (b) Comparison of the integrated intensity maps of $\mathrm{HCO}^{+}$emission from 12-m array data (gray image and black contours) and combined data (red contours). The contours are [0.1, $0.2,0.4,0.6,0.8] \times F_{\text {peak }}$. The peak integrated intensities $\left(F_{\text {peak }}\right)$ in $12-\mathrm{m}$ array data and in combined data are 0.93 and $0.96 \mathrm{Jy}_{\text {beam }}^{-1} \mathrm{~km} \mathrm{~s}^{-1}$, respectively. $(\mathrm{c})$ Same as in panel a but for $\mathrm{H}^{13} \mathrm{CO}^{+}$. (d) Same as in panel b but for $\mathrm{H}^{13} \mathrm{CO}^{+}$. The peak integrated intensities $\left(F_{\text {peak }}\right)$ in 12 -m array data and in combined data are the same as $0.75 \mathrm{Jy}_{\text {beam }}{ }^{-1} \mathrm{~km} \mathrm{~s}^{-1}$.

Table 3. Parameters of continuum sources in G9.62+0.19.

\begin{tabular}{|c|c|c|c|c|c|c|c|}
\hline \multirow[t]{2}{*}{ Name } & \multicolumn{4}{|c|}{$3 \mathrm{~mm}$ continuum } & \multicolumn{3}{|c|}{$\mathrm{H}_{40 \alpha}$} \\
\hline & size & $\begin{array}{c}I \\
\left(\mathrm{mJy} \mathrm{beam}^{-1}\right)\end{array}$ & $\begin{array}{c}S_{V} \\
(\mathrm{mJy})\end{array}$ & $\begin{array}{c}S_{\text {free-free }} \\
(\mathrm{mJy})\end{array}$ & $\begin{array}{c}I_{p}^{a} \\
\left(\mathrm{mJy} \mathrm{beam}^{-1}\right)\end{array}$ & $\begin{array}{c}V_{l s r} \\
\left(\mathrm{~km} \mathrm{~s}^{-1}\right)\end{array}$ & $\begin{array}{l}\text { FWHM } \\
\left(\mathrm{km} \mathrm{s}^{-1}\right)\end{array}$ \\
\hline B & $15.30^{\prime \prime} \times 13.31^{\prime \prime}\left(142^{\circ}\right)$ & 4.6 & 437 & 359 & 5.0 & 3.2 & 20.0 \\
\hline $\mathrm{MM} 1 / \mathrm{C}$ & $3.01^{\prime \prime} \times 2.10^{\prime \prime}\left(132^{\circ}\right)$ & 8.17 & 32.6 & 32 & 6.6 & 0.2 & 20.4 \\
\hline MM4/E. & $1.28^{\prime \prime} \times 1.02^{\prime \prime}\left(89^{\circ}\right)$ & 25.4 & 40.9 & $<2$ & $<3$ & & $<20$ \\
\hline MM6 & $1.75^{\prime \prime} \times 1.05^{\prime \prime}\left(173^{\circ}\right)$ & 5.4 & 10.4 & & & & \\
\hline MM7/G & & 5.7 & 5.7 & & & & \\
\hline MM8/F & $2.53^{\prime \prime} \times 1.28^{\prime \prime}\left(166^{\circ}\right)$ & 13.2 & 35.5 & $<3$ & $<3$ & & $<20$ \\
\hline MM11/D & $1.04^{\prime \prime} \times 0.74^{\prime \prime}\left(72^{\circ}\right)$ & 71.6 & 98.8 & 73 & 17.8 & 11.8 & 25.8 \\
\hline
\end{tabular}

${ }^{a}$ The values for MM4/E and MM8/F are upper limits based on rms level.

is clearly detected towards "B", "MM1/C" and "MM11/D", and is marginally detected toward MM4/E. Figure 6 presents the sourceaveraged $\mathrm{H}_{40 \alpha}$ spectra. The spectra were fitted with gaussian profiles, and the fit results (peak intensity $I_{p}$, systemic velocity $V_{\text {lsr }}$ and line width FWHM) are summarized in columns 6-8 of Table 3. The line widths of $\mathrm{H}_{40 \alpha}$ of "B" and "MM1/C" are $\sim 20 \mathrm{~km} \mathrm{~s}^{-1}$. "MM11/D" has the largest line width $\left(\sim 25 \mathrm{~km} \mathrm{~s}^{-1}\right)$ and velocity $\left(\sim 12 \mathrm{~km} \mathrm{~s}^{-1}\right)$ in $\mathrm{H}_{40 \alpha}$ line emission. The velocity $\left(\sim 12 \mathrm{~km} \mathrm{~s}^{-1}\right)$ of ionized gas in "MM11/D" is significantly redshifted relative to that $\left(\sim 4.5 \mathrm{~km} \mathrm{~s}^{-1}\right)$ of the molecular gas, indicating a fast flow in the ionized gas as also seen in the Hyper Compact (HC) HII region G24.78+0.08 (Moscadelli et al. 2018).

We estimated the free-free emission at $3 \mathrm{~mm}$ for "B", "MM1/C" and "MM11/D", which are listed in column 5 of Table 3 . An electron temperature of $\sim 6760 \mathrm{~K}$ is adopted, which is derived from the electron temperature gradient in the Galactic disk
(Quireza et al. 2006). Higher electron temperature will lead to higher free-free emission contributions. The electron temperature could be better constrained by observing multiple RRLs in future (Zhu et al. 2019). We found that the $3 \mathrm{~mm}$ continuum emission in "B", "MM1/C" and "MM11/D" is dominated by free-free emission, as suggested in previous works (Hofner et al. 1996). The free-free emission in other sources is negligible. Since the properties of all the continuum sources have been well discussed in Liu et al. (2017), we will not derive any other parameters from the $3 \mathrm{~mm}$ continuum emission in this work.

Fragmented massive filaments are commonly seen in infrared dark clouds (IRDCs; Zhang \& Wang 2011; Wang et al. 2011, 2012, 2014, 2016; Zhang et al. 2015; Sanhueza et al. 2019). Those massive filaments in IRDCs also fragment into a chain of dense cores as seen in G9.62+0.19. However, those filaments in IRDCs seem to be undergoing cylindrical fragmentation governed by turbulence 


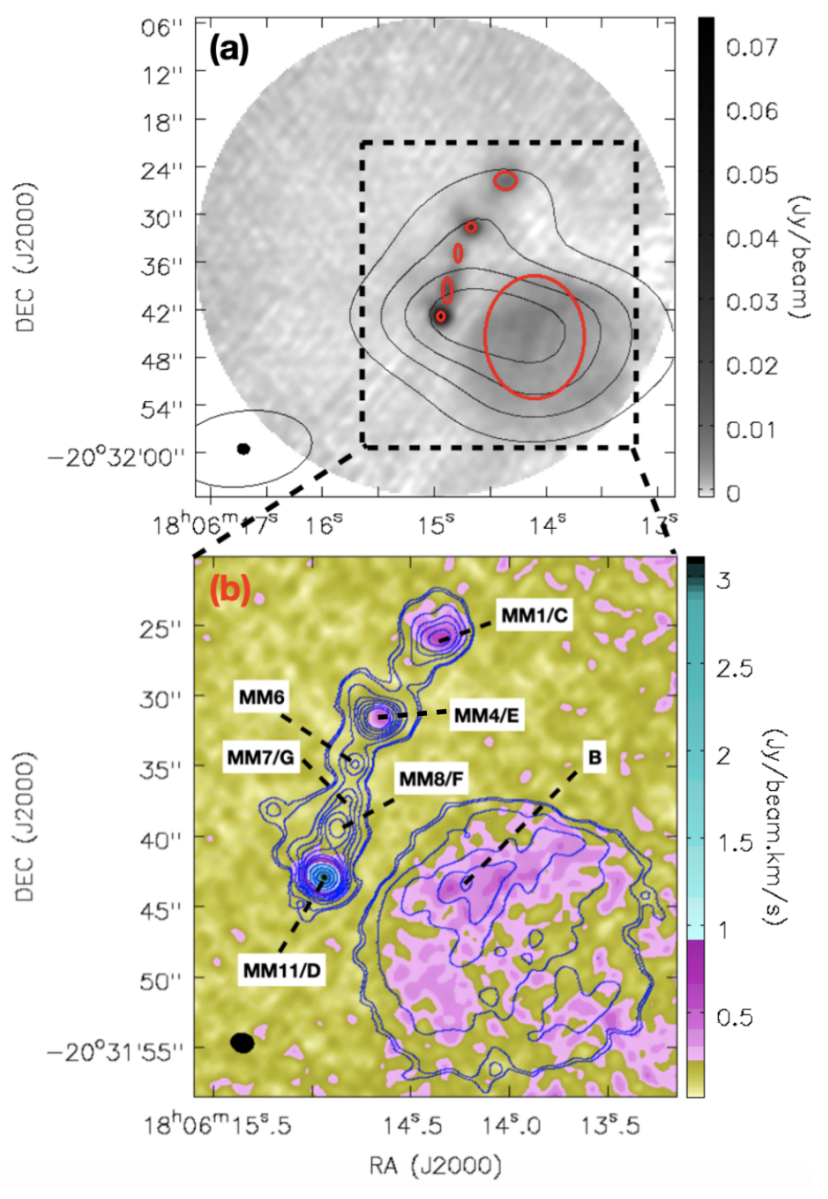

Figure 5. The $3 \mathrm{~mm}$ continuum emission of G9.62+0.19. (a) The 3 $\mathrm{mm}$ continuum emission from ACA observations is shown as contours at $[0.2,0.4,0.6,0.8] \times 0.225 \mathrm{Jy} \mathrm{beam}^{-1}$. The starting (faintest) contour corresponds to $\sim 10 \sigma\left(\sigma=4 \mathrm{mJy}^{\text {beam }}{ }^{-1}\right)$. The background image is the $3 \mathrm{~mm}$ continuum emission from the 12-m array observations. The red ellipses show the continuum sources identified with FellWalker. (b) The $3 \mathrm{~mm}$ continuum emission from 12-m array observations is shown as blue contours with contour levels of $[0.01,0.013,0.03,0.05,0.07,0.1,0.15,0.2,0.4,0.6,0.8] \times 74.1 \mathrm{mJy}_{\text {beam }}{ }^{-1}$. The starting (faintest) contour corresponds to $\sim 3.5 \sigma\left(\sigma=0.2 \mathrm{mJy}^{\text {beam }}{ }^{-1}\right)$. The background (colored) image is the intensity of the $\mathrm{H}_{40 \alpha}$ line integrated from -50 to $50 \mathrm{~km} \mathrm{~s}^{-1}$. The names (from Liu et al. 2017) of seven main components in the $3 \mathrm{~mm}$ continuum emission are indicated in the image.

(Wang et al. 2011, 2014). In contrast, the fragmentation of the massive filament in G9.62+0.19 can be well explained by thermal Jeans instability (Liu et al. 2017).

Within the ATOMS project, we will systematically investigate the fragmentation process of proto-clusters as G9.62+0.19, and compare with IRDC clumps. This will help figure out the dominating mechanisms in dense-core formation in different Galactic environments and conditions.

\subsection{Spatial distribution of molecules}

One of the main science goals of the ATOMS project is to investigate the small-scale distributions of various molecular gas tracers within massive star forming regions. In particular, we will be more focused on those dense gas tracers, e.g., $J=1-0$ of $\mathrm{HCN}$ and $\mathrm{HCO}^{+}$,

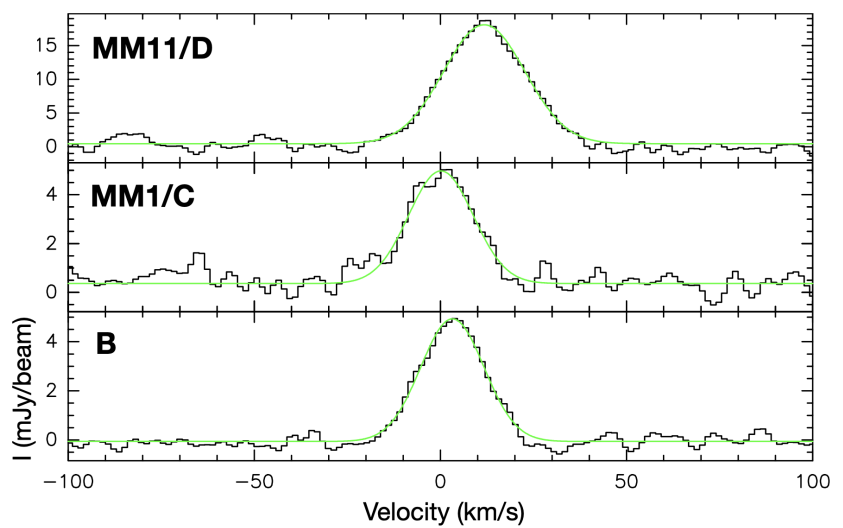

Figure 6. Source-averaged $\mathrm{H}_{40 \alpha}$ RRL in G9.62+0.19. The green lines are gaussian fits.

and $J=2-1$ of CS, which are commonly used in studying star formation scaling relations. By comparing with observational results in nearby clouds (Kauffmann et al. 2017; Pety et al. 2017; Shimajiri et al. 2017), we will investigate how well those gas tracers unveil the structures and masses of molecular clumps that are located in different Galactic environments and have different physical conditions, e.g., density, temperature, and UV radiation.

\subsubsection{Gas clumps in ACA observations of G9.62+0.19}

The ACA observations have large FOV $\left(\sim 2^{\prime}\right)$ and maximum recovering scale (MRS; $\sim 1^{\prime}$ ), enabling us to study the overall properties of gas emission at clump scale, such as total line luminosity, emission extent, and virial masses (Liu et al. 2020). Figure 7 presents the integrated intensity (from 1.5 to $6.5 \mathrm{~km} \mathrm{~s}^{-1}$ ) maps of various gas tracers in ACA observations of G9.62+0.19. All the gas tracers reveal a single gas clump that can be well modeled by a 2D Gaussian profile. We performed 2D Gaussian fitting in the gas emission regions bounded by the $20 \%$ contour of their peak values. From 2D Gaussian fitting (see section 3.4), we found that the spatial distributions of the emission in all lines are smaller than the MRS $\left(\sim 1^{\prime}\right)$ in ACA observations. In addition, except $\mathrm{HCN}$ and $\mathrm{HCO}^{+}$, the other lines have FWHM angular sizes smaller than $20^{\prime \prime}$, suggesting that 12 -m array observations with MRS $\sim 20^{\prime \prime}$ could recover most of the line flux.

In a companion work (Liu et al. 2020), we report studies of the star formation scaling relationships between the total bolometric emission $\left(L_{\mathrm{bol}}\right.$ or $\left.L_{\mathrm{TIR}}\right)$ and the molecular line luminosities of $\mathrm{J}=1-0$ transitions of $\mathrm{H}^{13} \mathrm{CN}, \mathrm{H}^{13} \mathrm{CO}^{+}, \mathrm{HCN}$, and $\mathrm{HCO}^{+}$with data obtained from ACA observations for the whole "ATOMS" sample. We found that both main lines and isotopologue lines can well reveal the total masses of dense gas in Galactic molecular clumps.

However, although they are all good tracers of total masses of dense gas, their spatial distributions may be different. The upper panel of Figure 8 shows the $3 \mathrm{~mm}$ continuum emission in colorscale overlaid with $\mathrm{H}^{13} \mathrm{CO}^{+}$integrated intensity map in contours. The emission peaks of various gas tracers as revealed in the ACA observations of G9.62+0.19 are marked on this plot. The emission peaks of most gas tracers (except $\mathrm{HCN}, \mathrm{HCO}^{+}$and $\mathrm{H}_{40 \alpha}$ ) are close to the $\mathrm{H}^{13} \mathrm{CO}^{+}$emission peak. The ionized gas traced by $\mathrm{H}_{40 \alpha}$ is well accociated with the $3 \mathrm{~mm}$ continuum emission but is clearly offset from molecular gas and peaks to the south-west. The emission peaks of $\mathrm{HCN}$ and $\mathrm{HCO}^{+}$are located to the north-west of the 


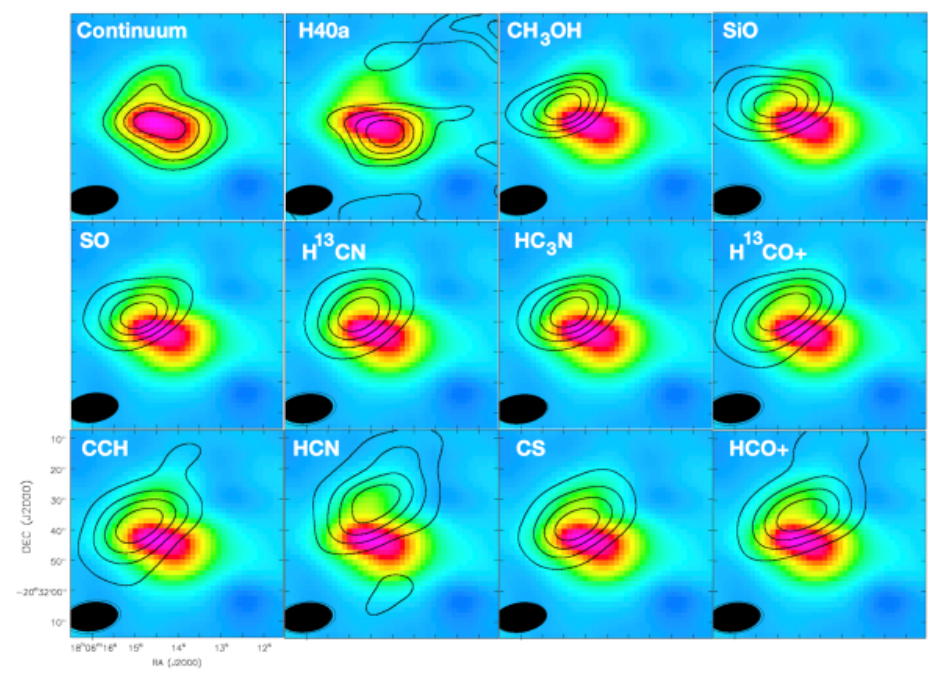

Figure 7. The integrated intensity maps from the ACA images of molecular line emission in G9.62+0.19 are shown in contours. The background color image is the $3 \mathrm{~mm}$ continuum emission. The contour levels are $[0.2,0.4,0.6,0.8] \times F_{\text {peak }}$. The $F_{\text {peak }}$ for various tracers are: Continuum: $0.225 \mathrm{Jy}$ beam ${ }^{-1} ; \mathrm{H}_{40 \alpha}: 7.8$ Jy beam ${ }^{-1} \mathrm{~km} \mathrm{~s}^{-1} ; \mathrm{CH}_{3} \mathrm{OH}: 10.3$ Jy beam ${ }^{-1} \mathrm{~km} \mathrm{~s}^{-1}$; SiO: $10.3 \mathrm{Jy}_{\text {beam }}{ }^{-1} \mathrm{~km} \mathrm{~s}^{-1}$; SO: $28.8 \mathrm{Jy}_{\text {beam }}{ }^{-1} \mathrm{~km} \mathrm{~s}^{-1} ; \mathrm{H}^{13} \mathrm{CN}^{-1}: 3.3 \mathrm{Jy} \mathrm{beam}^{-1} \mathrm{~km} \mathrm{~s}^{-1} ; \mathrm{HC}_{3} \mathrm{~N}$ : $34.5 \mathrm{Jy} \mathrm{beam}^{-1} \mathrm{~km} \mathrm{~s}^{-1} ; \mathrm{H}^{13} \mathrm{CO}^{+}$: $15.0 \mathrm{Jy}$ beam ${ }^{-1} \mathrm{~km} \mathrm{~s}^{-1}$; CCH: $13.0 \mathrm{Jy}$ beam ${ }^{-1} \mathrm{~km} \mathrm{~s}^{-1}$; HCN: $12.1 \mathrm{Jy} \mathrm{beam}^{-1} \mathrm{~km} \mathrm{~s}^{-1}$; CS: $53.9 \mathrm{Jy}$ beam ${ }^{-1} \mathrm{~km} \mathrm{~s}^{-1}$; $\mathrm{HCO}^{+}: 23.6 \mathrm{Jy}_{\text {beam }}^{-1} \mathrm{~km} \mathrm{~s}^{-1}$.

$\mathrm{H}^{13} \mathrm{CO}^{+}$emission peak, indicating that the main lines may show different spatial distributions from their isotopologue lines. The detailed spatial distributions of various gas tracers can be investigated with the high resolution 12-m array data.

\subsubsection{Moment maps in 12-m array observations}

In the lower panel of Figure 8, we compare the $\mathrm{H}^{13} \mathrm{CO}^{+}$spectra from ACA and 12-m array observations. The spectra that were averaged over $30^{\prime \prime}$ region centered at the emission peak are nearly identical in both observations. The 12-m array observation recovers more than $92 \%$ of $\mathrm{H}^{13} \mathrm{CO}^{+}$flux in ACA observations. From gaussian fits to the spectra, we find a systemic velocity of $\sim 4 \mathrm{~km} \mathrm{~s}^{-1}$. The line width is about $5 \mathrm{~km} \mathrm{~s}^{-1}$. To compare the spatial distributions of various gas tracers (see Table 2) revealed in 12-m array observations, we integrate their intensity in the same velocity interval that is from 1.5 to $6.5 \mathrm{~km} \mathrm{~s}^{-1}$ above a threshold of $3 \sigma$. This velocity interval is chosen based on the line width of $\mathrm{H}^{13} \mathrm{CO}^{+}$to avoid contamination from outflow emission. In Figures A1, A2 and A3, their integrated intensity (Moment 0) maps (left panels), intensityweighted velocity (Moment 1) maps (middle panels) and intensityweighted velocity dispersion (Moment 2) maps (right panels) are presented as color images. The moment maps were constructed from the data after imposing a cutoff of $3 \sigma$. The $3 \mathrm{~mm}$ continuum emission is shown as contours on the moment 0 maps for comparison. The contours on moment 1 and 2 maps are showing the integrated intensity maps of corresponding line emission. Below we will discuss the detailed spatial distributions of various gas tracers based on these maps.

\subsubsection{Extended gas emission}

$\mathrm{CS}, \mathrm{HCN}$ and $\mathrm{HCO}^{+}$are commonly said to be dense gas tracers used in determinations of dense gas star formation laws (Gao \& Solomon 2004; Wu et al. 2005, 2010), but this is not always true (Kauffmann et al. 2017; Pety et al. 2017; Shimajiri et al. 2017). As shown in Figure A1, all the three tracers show very extended emission in the G9.62+0.19 complex. They are poor tracers of the massive filament, where new high-mass stars are forming. Due to large optical depths or absorption, their moment 1 and moment 2 maps are very complicated and do not show any clear pattern. HCN emission reveals a dense shell-like structure with its emission peak at the position "MM4/E". What we have learned here is that opaque tracers will fail to trace the locations of densest gas on small scales $(\sim 0.1 \mathrm{pc})$ though they are sort of fine to reveal the overall dense gas distribution on larger scales $(\sim 1 \mathrm{pc}$; see Figure 7$)$.

Besides these main lines with low effective excitation densities $\left(n_{\text {eff }}<5 \times 10^{3} \mathrm{~cm}^{-3}\right)$, the lines with higher effective excitation densities $\left(n_{\text {eff }}>1 \times 10^{4} \mathrm{~cm}^{-3}\right)$ in Table 2 including the isotopologue lines $\left(\mathrm{H}^{13} \mathrm{CO}^{+}, \mathrm{H}^{13} \mathrm{CN}\right)$ also show widespread emission (see Figures A2 and A3). Interestingly, the HII region "B" appears to have produced a cavity in the molecular clump, which is clearly seen in $\mathrm{CCH}, \mathrm{H}^{13} \mathrm{CO}^{+}, \mathrm{H}^{13} \mathrm{CN}$, SO and $\mathrm{HC}_{3} \mathrm{~N}$ emission. The HII region " $\mathrm{B}$ " is bounded by molecular line emission. $\mathrm{H}^{13} \mathrm{CN}$ emission and $3 \mathrm{~mm}$ continuum emission together define a nearly circular region that has a radius of $\sim 0.5 \mathrm{pc}$, as shown by the magenta dashed circles in Figures A1, A2 and A3. It seems that all high-mass stars in G9.62+0.19 are formed within this $1 \mathrm{pc}$ size natal clump. The Moment 1 maps of these molecular line emission show a clear velocity gradient in the southwest to northeast direction. The gas close to the HII region "B" shows higher redshifted emission. Away from the HII region "B", the gas radial velocity decreases. Line profiles closer to the boundary of the HII region "B" are more redshifted, indicating that the HII region "B" is expanding into its surrounding molecular gas and is reshaping its natal clump.

\subsubsection{Tracers for photodissociation regions}

$\mathrm{CCH}$ is a good tracer of photodissociation regions (PDRs; Pety et al. 2005; Tiwari et al. 2019). In PDRs, the fragmentation of polycyclic aromatic hydrocarbons (PAHs) due to FUV radiation (Le Page et al. 2003; Pety et al. 2005; Montillaud et al. 2013) or new gas-phase formation routes (Cuadrado et al. 2015) facilitated by heating from high UV flux $\left(\mathrm{G}_{0} \sim 10^{4}-10^{5}\right.$ in Habing units) could 

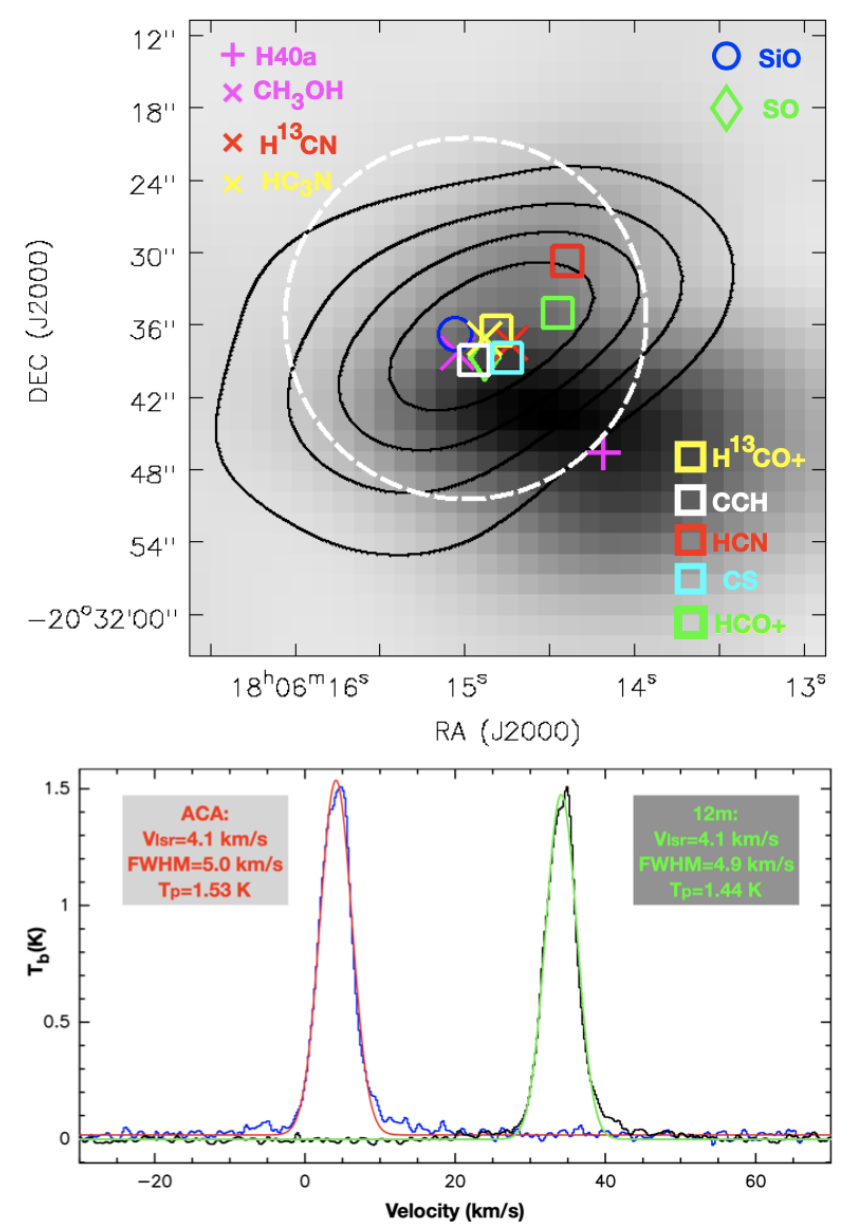

Figure 8. (a) The $3 \mathrm{~mm}$ continuum emission of G9.62+0.19 in ACA observations is shown as the grey-scale. The integrated intensity from 1.5 to 6.5 $\mathrm{km} \mathrm{s}^{-1}$ of $\mathrm{H}^{13} \mathrm{CO}^{+}$is shown as contours at levels of $[0.2,0.4,0.6,0.8] \times 15$ $\mathrm{Jy} \mathrm{beam}^{-1} \mathrm{~km} \mathrm{~s}^{-1}$. The peak positions of various gas tracers of G9.62+0.19 derived from ACA observations are marked with different symbols. (b) The $\mathrm{H}^{13} \mathrm{CO}^{+}$spectra from the 12-m array and ACA averaged over a $30^{\prime \prime}$ diameter region (white dashed circle in panel a) are displayed in blue and black, respectively. The spectrum from $12-\mathrm{m}$ array data is shifted by $30 \mathrm{~km} \mathrm{~s}^{-1}$. The red and green curves are gaussian fits. The results of gaussian fits are also displayed on the panel.

give rise to a rich hydrocarbon chemistry. However, $\mathrm{CCH}$ emission is widespread in the G9.62+0.19. As shown in Figure A2, the $\mathrm{CCH}$ emission shows a morphology remarkably similar to that of the $\mathrm{H}^{13} \mathrm{CO}^{+}$emission. It indicates that $\mathrm{CCH}$ emission inside massive clumps is not only concentrated in PDRs but is almost omnipresent. $\mathrm{CCH}$ has been detected in both nearby dark clouds (Pratap et al. 1997) and distant high-mass star forming clumps (Beuther et al. 2008; Sanhueza et al. 2012). CCH is produced quickly early on and gets replenished at the clump surfaces by the UV photodissociation of CO (Beuther et al. 2008), leading to its widespread emission in clouds.

$\mathrm{HCO}^{+}$is more easily recombined with free electrons and its abundance is sensitive to the ionization degree of molecular gas. In far-UV irradiated environments (PDRs), its abundances may decrease. Figure 9a presents the $\mathrm{CCH}-$ to- $\mathrm{H}^{13} \mathrm{CO}^{+}$integrated intensity ratio map. In the interface between the HII region " $\mathrm{B}$ " and the massive filament, the $\mathrm{CCH}-$ to- $\mathrm{H}^{13} \mathrm{CO}^{+}$intensity ratio is enhanced
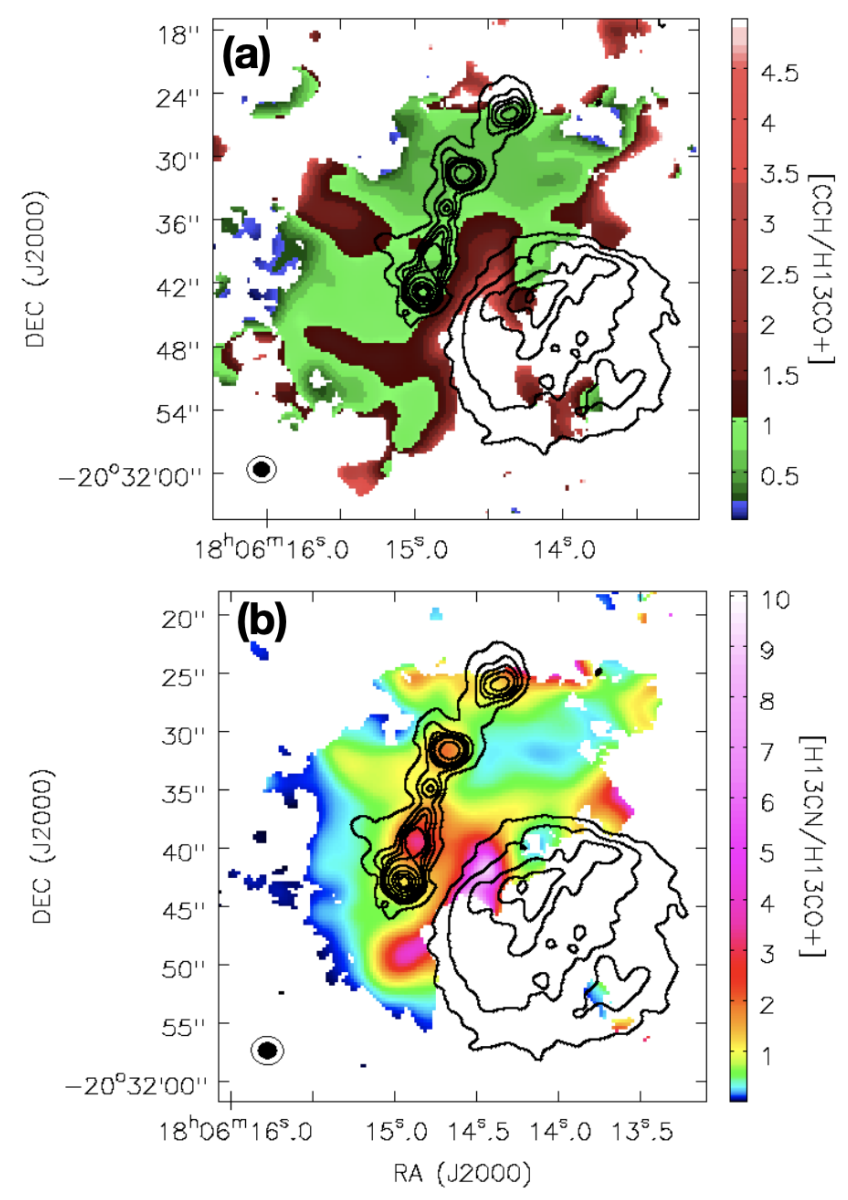

Figure 9. Integrated intensity ratio maps of G9.62+0.19. The contours show the $3 \mathrm{~mm}$ continuum emission. Contour levels are the same as in Figure 5. (a) $\mathrm{CCH}$-to- $\mathrm{H}^{13} \mathrm{CO}^{+}$ratio; (b) $\mathrm{H}^{13} \mathrm{CN}$-to- $\mathrm{H}^{13} \mathrm{CO}^{+}$ratio.

by a factor of 2-5 when compared to the massive filament itself and its surrounding regions. The enhanced $\mathrm{CCH}-\mathrm{to}-\mathrm{H}^{13} \mathrm{CO}^{+}$intensity ratio is likely caused by the PDR of the HII region " $\mathrm{B}$ ". The $\mathrm{H}^{13} \mathrm{CN}$-to- $\mathrm{H}^{13} \mathrm{CO}^{+}$ratio is also enhanced in this thin layer, as seen in Figure 9b. High $\mathrm{HCN}$-to- $\mathrm{HCO}^{+}$ratios have also been found in far-UV irradiated environments such as evolved Galactic HII regions (Nguyen-Luong et al. 2020), AGNs (Aladro et al. 2015) or luminous infrared galaxies (LIRGs; Papadopoulos 2007). Therefore, it implies that the high $\mathrm{CCH}-$ to- $\mathrm{H}^{13} \mathrm{CO}^{+}$and $\mathrm{H}^{13} \mathrm{CN}$ to- $\mathrm{H}^{13} \mathrm{CO}^{+}$ratios are good tracers of PDRs.

In the ATOMS survey, we will reveal the PDRs utilizing the $\mathrm{CCH}$-to- $\mathrm{H}^{13} \mathrm{CO}^{+}$and $\mathrm{H}^{13} \mathrm{CN}$-to- $\mathrm{H}^{13} \mathrm{CO}^{+}$ratios. We will systematically investigate the chemistry and structure of PDRs (e.g., Goicoechea et al. 2016). In particular, we will study how PDRs interact with their natal molecular clumps. In the G9.62+0.19 complex, the PDR of the HII region "B" is clearly interacting with its molecular clump as indicated by the velocity gradients in Moment 1 maps of $\mathrm{CCH}, \mathrm{H}^{13} \mathrm{CO}^{+}, \mathrm{H}^{13} \mathrm{CN}$, SO and $\mathrm{HC}_{3} \mathrm{~N}$ emission (see Figures $\mathrm{A} 2$ and $\mathrm{A} 3$ ).

\subsubsection{Widespread shocked gas emission}

In the ATOMS survey, several shock tracers, e.g., $\mathrm{SO}, \mathrm{SiO}$ and $\mathrm{CH}_{3} \mathrm{OH}$, are observed and will be used to trace the shocked gas 
Table 4. Parameters of $\mathrm{SiO}$ lines toward G9.62+0.19.

\begin{tabular}{cccc}
\hline Position & $\begin{array}{c}I_{p} \\
\left(\mathrm{mJy} \mathrm{beam}^{-1}\right)\end{array}$ & $\begin{array}{c}V_{l s r} \\
\left(\mathrm{~km} \mathrm{~s}^{-1}\right)\end{array}$ & $\begin{array}{c}\text { FWHM } \\
\left(\mathrm{km} \mathrm{s}^{-1}\right)\end{array}$ \\
\hline 1 & 39 & $3.38(0.05)$ & $2.25(0.10)$ \\
2 & 10 & $0.94(0.25)$ & $3.70(0.93)$ \\
& 14 & $3.53(0.49)$ & $11.18(0.64)$ \\
3 & 16 & $2.29(0.10)$ & $2.53(0.25)$ \\
& 11 & $4.21(0.30)$ & $9.55(0.77)$ \\
4 & 44 & $3.87(0.03)$ & $2.73(0.08)$ \\
7 & 39 & $6.12(0.19)$ & $6.12(0.27)$ \\
& 13 & $12.62(0.82)$ & $7.78(1.36)$ \\
8 & 30 & $-3.30(0.22)$ & $6.97(0.30)$ \\
& 54 & $1.07(0.06)$ & $3.80(0.11)$ \\
9 & 36 & $1.92(0.06)$ & $5.13(0.14)$ \\
\hline
\end{tabular}

emission. As shown in Figure $\mathrm{A} 2, \mathrm{SiO}$ emission is widespread in the G9.62+0.19 region. Within the $20 \%$ contour in its integrated intensity map, $\mathrm{SiO}$ emission shows a large velocity dispersion $\left(\sigma>1.5 \mathrm{~km} \mathrm{~s}^{-1}\right)$, which is mainly related to outflows. However, in more extended regions, i.e., outside the $20 \%$ contour in integrated intensity map, $\mathrm{SiO}$ emission has a much smaller velocity dispersion of $<1 \mathrm{~km} \mathrm{~s}^{-1}$. In Figure 10, we present the Moment 2 map of $\mathrm{SiO}$ emission in the upper-left panel and plot the $\mathrm{SiO}$ spectra at nine positions of G9.62+0.19 region in other panels. The $\mathrm{SiO}$ spectra were obtained by averaging over $5^{\prime \prime}$ area at each position. At positions 5 and $6, \mathrm{SiO}$ shows broad line emission with high-velocity wings, indicating that $\mathrm{SiO}$ in these regions is affected by outflow shocks. At other positions, however, $\mathrm{SiO}$ emission has much narrower line widths. We fit the spectra with gaussian profiles and the results (peak intensity $\mathrm{I}_{P}$, systemic velocity $\mathrm{V}_{l s r}$ and line width FWHM) from gaussian fits of the spectra are summarized in Table 4. The positions 1-4 are located close to HII regions ("B", "MM1/C", "MM4/E") and $\mathrm{SiO}$ could be excited by HII region shocks. Positions 7-9 are away from both outflows and HII regions. $\mathrm{SiO}$ emission at these positions may be caused by other mechanisms, e.g., shocks from large-scale colliding flows. Spectra at positions 1,4 and 9 can be well fitted with a single gaussian profile, while spectra at positions $2,3,7$, and 8 are better fitted with two gaussians including one narrow component and one broad component. The line widths of the narrow components range from $2 \mathrm{~km} \mathrm{~s}^{-1}$ to $6 \mathrm{~km} \mathrm{~s}^{-1}$. Widespread, narrow $\mathrm{SiO}$ emission has been detected in infrared dark clouds (Jiménez-Serra et al. 2010; Cosentino et al. 2018, 2019) and high-mass proto-cluster forming regions (Nguyen-Lu'o'ng et al. 2013; Louvet et al. 2016). Such narrow $\mathrm{SiO}$ emission can be reproduced by low-velocity shocks in the range $7 \mathrm{~km} \mathrm{~s}^{-1}$ to $12 \mathrm{~km} \mathrm{~s}^{-1}$ (Louvet et al. 2016). Such lowvelocity shocks are usually attributed to colliding flows or cloudcloud collision (Jiménez-Serra et al. 2010; Nguyen-Lu'o'ng et al. 2013; Louvet et al. 2016; Moscadelli et al. 2018). We noticed that the narrow components of $\mathrm{SiO}$ spectra at positions 7 and 8 are clearly redshifted and blueshifted by $\sim 2-3 \mathrm{~km} \mathrm{~s}^{-1}$ from the systemic velocity $\left(4 \mathrm{~km} \mathrm{~s}^{-1}\right.$ measured from $\left.\mathrm{H}^{13} \mathrm{CO}^{+}\right)$with a projected velocity difference of $\sim 5 \mathrm{~km} \mathrm{~s}^{-1}$, while the wider components of $\mathrm{SiO}$ spectra at positions 7 and 8 are redshifted and blueshifted by $\sim 7-9 \mathrm{~km} \mathrm{~s}^{-1}$ from the systemic velocity with a projected velocity difference of $\sim 16 \mathrm{~km} \mathrm{~s}^{-1}$. This velocity difference indicates that the two elongated structures associated with positions 7 and 8 could be related to large-scale colliding flows that induce lowvelocity shocks.

$\mathrm{CH}_{3} \mathrm{OH}$ and SO may also trace shocked gas. The upper six panels of Figure A3 present the moment maps for $\mathrm{CH}_{3} \mathrm{OH}$ and SO. In outflow regions, $\mathrm{CH}_{3} \mathrm{OH}$ and $\mathrm{SO}$ emission show a large velocity dispersion, indicating that they also trace outflow shocks. Beyond the outflow and filament regions, however, the velocity dispersion of $\mathrm{CH}_{3} \mathrm{OH}$ and $\mathrm{SO}$ is as small $\left(<1 \mathrm{~km} \mathrm{~s}^{-1}\right)$ as that of $\mathrm{SiO}$, suggesting $\mathrm{CH}_{3} \mathrm{OH}$ and $\mathrm{SO}$ may also trace widespread low-velocity shocks generated from either large-scale colliding flows or HII regions. Widespread $\mathrm{CH}_{3} \mathrm{OH}$ emission that may have originated in a large-scale shock interaction has also been detected in infrared dark clouds (Cosentino et al. 2018). However, $\mathrm{CH}_{3} \mathrm{OH}$ and $\mathrm{SO}$ emission can not only be induced by shocks but also be excited due to pure gas-chemistry in cold cores (Spezzano et al. 2017; Nagy et al. 2019) or hot cores (Esplugues et al. 2013; Qin et al. 2015). Indeed, they reveal well the dense cores in G9.62+0.19 too (see section 3.2.6).

In the ATOMS survey, we will for the first time investigate statistically the origin of various velocity shocks in a large sample of massive clumps.

\subsubsection{Gas emission tracing dense cores}

Figure A3 presents moment maps of four gas tracers, $\mathrm{CH}_{3} \mathrm{OH}, \mathrm{SO}$, $\mathrm{H}^{13} \mathrm{CN}$ and $\mathrm{HC}_{3} \mathrm{~N}$. All of them trace well the dense cores in the massive filament as identified by the $3 \mathrm{~mm}$ continuum emission. $\mathrm{H}^{13} \mathrm{CN}$ and $\mathrm{HC}_{3} \mathrm{~N}$ have very similar morphology. Within the $20 \%$ contours in their integrated intensity maps, their morphology is quite similar to that of the $3 \mathrm{~mm}$ continuum emission. When compared with other gas tracers in Table 2, these four molecular line transitions seem to be better tracers of dense cores as well as tracers of the massive filament. Their emission maps agree with the 3 $\mathrm{mm}$ continuum more than the maps of $\mathrm{HCN}$ and $\mathrm{HCO}^{+}$.

\subsubsection{Outflow gas emission}

Several gas tracers in the ATOMS survey, including $\mathrm{SiO}, \mathrm{SO}, \mathrm{CS}$, $\mathrm{HCN}, \mathrm{HCO}^{+}$and $\mathrm{CH}_{3} \mathrm{OH}$, can trace outflows. Here we demonstrate that the ATOMS data are very useful for identifying protostellar outflows. In Figure 11, we present the redshifted and blueshifted high-velocity emission of these tracers. The HCN line has three hyper-fine components. To avoid contamination of hyperfine component emission from dense cores, the velocity intervals for redshifted and blueshifted high-velocity emission of HCN are [20, 50] $\mathrm{km} \mathrm{s}^{-1}$ and $[-42,-8] \mathrm{km} \mathrm{s}^{-1}$, respectively. For the other tracers, the velocity intervals for redshifted and blueshifted high-velocity emission are $[9,50] \mathrm{km} \mathrm{s}^{-1}$ and $[-42,-1] \mathrm{km} \mathrm{s}^{-1}$, respectively. $\mathrm{CH}_{3} \mathrm{OH}$ traces part of the high-velocity outflows driven by the hot core MM8/F, but does not trace the high velocity outflows driven by the young high-mass proto-stellar object (HMPO) MM6 (Liu et al. 2017). The other five outflow tracers reveal the high-velocity outflows driven by both MM8/F and MM6. They show similar outflow morphologies. The outflow properties in G9.62+0.19 have been discussed in detail in Liu et al. (2017). In this paper, we will not further analyze the outflows.

\subsubsection{Chemical differentiation among dense cores}

The ATOMS survey covers a wide frequency range that includes many molecular transitions in the upper side-band. Figure 12 presents the observed spectra (in black) at the emission peak of G9.62+0.19 core MM8/F overlaid with simulated spectra (in red) from XCLASS. Several tens of molecular line transitions were 

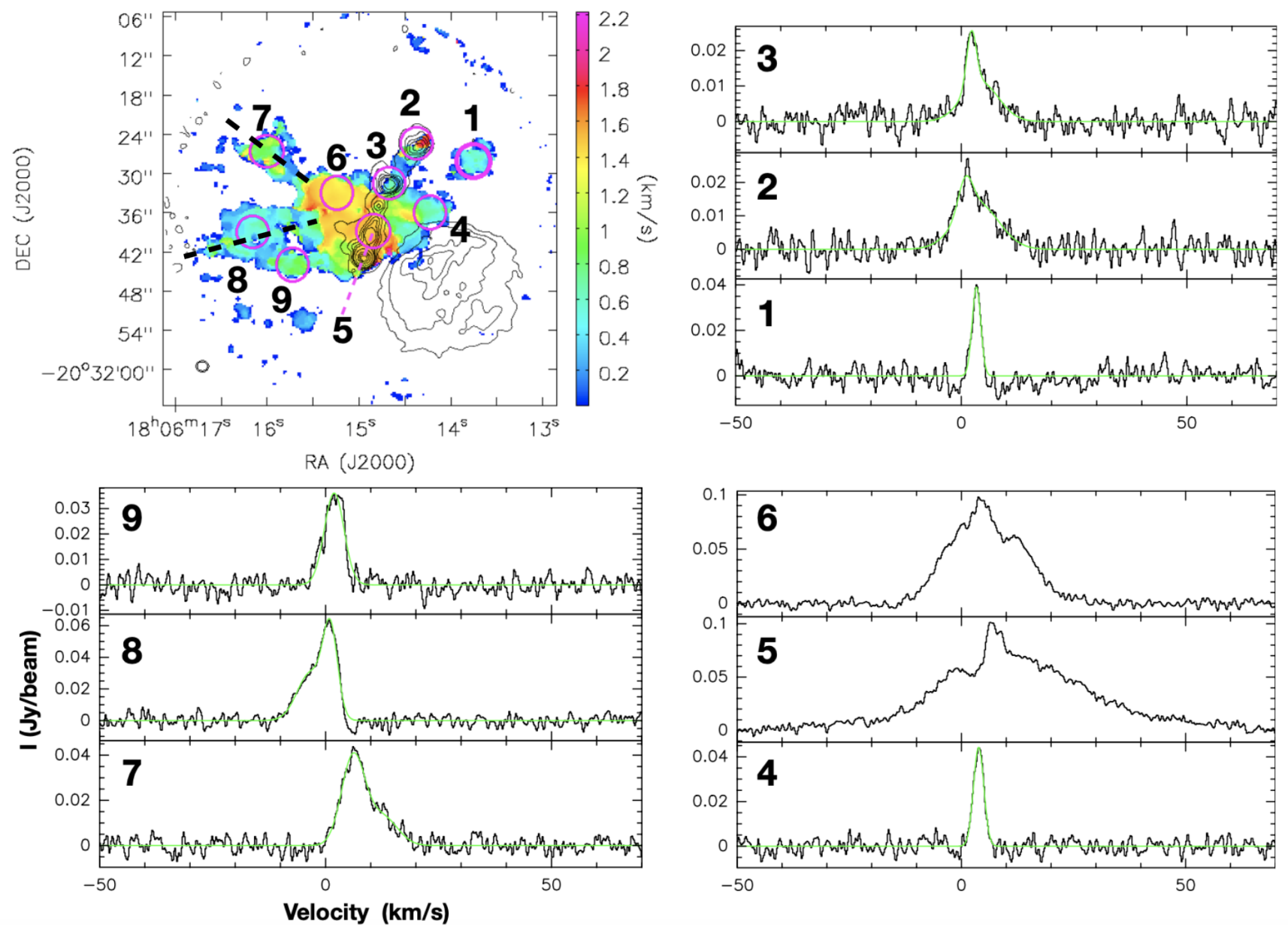

Figure 10. $\mathrm{SiO}$ spectra averaged over $5^{\prime \prime}$ regions at nine positions across G9.62+0.19. The upper left panel shows the Moment 2 map of SiO overlaid on 3 mm continuum emission contours. The contour levels are the same as in Figure 5. The magenta circles mark the positions where the SiO spectra are extracted. The two black dashed lines mark the elongated structures that may be related to colliding flows. The other panels show the averaged SiO spectra at each position. The green lines are gaussian fits.

identified toward MM8/F. Hence, the ATOMS data are very useful for studying the chemical properties of dense cores. We will identify spectral line transitions using the eXtended CASA Line Analysis Software Suite (XCLASS Möller et al. 2017), which takes the beam dilution, the line opacity, and line blending into account to identify and simulate the observed molecular line transitions under the assumption of local thermodynamical equilibrium (LTE). XCLASS has been widely used in chemistry studies in ALMA era (e.g., Peng et al. 2017, 2019; Allen et al. 2017; Pols et al. 2018). The detailed chemical analysis in G9.62+0.19 is beyond the scope of this paper and will be presented in a forthcoming paper (Peng, Y.-P. et al., 2020, in preparation).

The ATOMS survey data are also very useful for studying chemical differentiation among dense cores that are at different evolutionary stages. Figure 13 compares the spectra of the two wide spectral windows at the positions of six cores in G9.62+0.19. Chemical differentiation is clear among these cores. The cometary HII region "B" is the most evolved source in G9.62+0.19 that has ionized its gas and shows no molecular gas emission. MM1/C is also a cometary HII region but is younger than "B". Some strong molecular lines such as $\mathrm{HC}_{3} \mathrm{~N}$, SO and $\mathrm{CS}$ are detected toward it. MM11/D is an UC HII region, which shows strong $\mathrm{H}_{40 \alpha}$ emission. The molecular line emission toward MM11/D is also much stronger than the corresponding emission toward MM1/C. Cores MM4/E, MM7/G and MM8/F show typical hot core chemistry with line forests in the two wide bands. MM8/F is associated with energetic outflows and has the richest line emission. MM7/G is also an outflow source but may be still at an earlier evolutionary stage than MM8/F (Liu et al. 2017). Its line emission is much weaker than that of MM8/F. MM4/E is a hyper-compact (HC) HII region and is not associated with outflows (Liu et al. 2017). Its molecular line emission is also weaker than MM8/F. MM6 is the youngest high-mass protostellar object (HMPO) in this region that is driving an energetic bipolar outflow, and has not shown hot core chemistry.

Here we simply demonstrate that the ATOMS data are very useful for studying chemical differentiation among dense cores. In future, we will systematically investigate how the chemistry of dense cores changes with other physical properties like temperature, density, luminosity and Galactic environments.

\subsection{Similarities and differences in the spatial distributions of gas tracers}

Due to their different excitation conditions and abundances, different molecular line transitions may trace various physical conditions within molecular clouds, such as density, chemistry, temperature, 


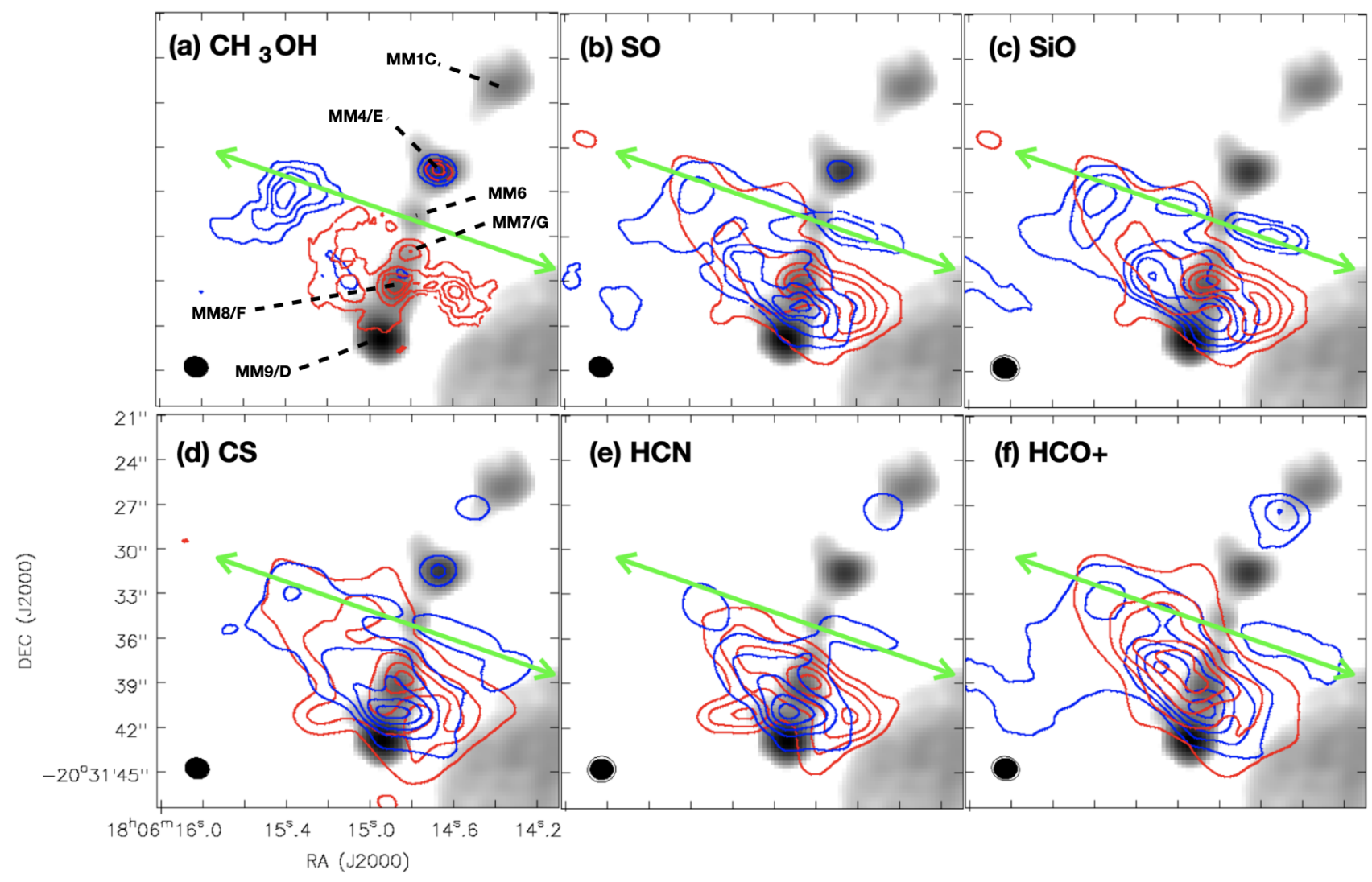

Figure 11. Redshifted and blueshifted high-velocity emission of various gas tracers of G9.62+0.19 are shown in contours. The contours are $[0.1,0.3,0.5,0.7,0.9] \times F_{\text {peak }}$. The peak integrated intensities $\left(F_{\text {peak }}\right)$ of $\mathrm{CH}_{3} \mathrm{OH}$ blueshifted and redshifted emission are $0.42 \mathrm{Jy} \mathrm{beam}^{-1} \mathrm{~km} \mathrm{~s}^{-1}$ and 0.32 Jy beam ${ }^{-1} \mathrm{~km} \mathrm{~s}^{-1}$, respectively; $F_{\text {peak }}$ of SO blueshifted and redshifted emission are $1.24 \mathrm{Jy}$ beam km s${ }^{-1}$ and $2.56 \mathrm{Jy}$ beam ${ }^{-1} \mathrm{~km} \mathrm{~s}^{-1}$, respectively; $F_{\text {peak }}$ of

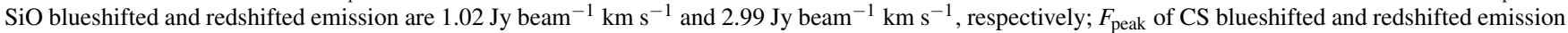
are: $3.89 \mathrm{Jy} \mathrm{beam}{ }^{-1} \mathrm{~km} \mathrm{~s}^{-1}$ and $6.6 \mathrm{Jy}$ beam ${ }^{-1} \mathrm{~km} \mathrm{~s}^{-1}$, respectively; $F_{\text {peak }}$ of HCN blueshifted and redshifted emission are: $5.80 \mathrm{Jy} \mathrm{beam}^{-1} \mathrm{~km} \mathrm{~s}^{-1}$ and 3.94 Jy beam ${ }^{-1} \mathrm{~km} \mathrm{~s}^{-1}$, respectively; $F_{\text {peak }}$ of $\mathrm{HCO}^{+}$blueshifted and redshifted emission are $1.72 \mathrm{Jy} \mathrm{beam}^{-1} \mathrm{~km} \mathrm{~s}^{-1}$ and $3.41 \mathrm{Jy} \mathrm{beam}^{-1} \mathrm{~km} \mathrm{~s}^{-1}$, respectively. The green arrow shows the direction of the bipolar outflow driven by MM6 (Liu et al. 2017). The outflows to the south are mainly driven by MM8/F (Liu et al. 2011, 2017). The background image shows the $3 \mathrm{~mm}$ continuum emission.

UV radiation and so on. Through studying the similarities and differences in the spatial distributions of various gas tracers, we could figure out the best tracers for different structures inside molecular clouds, e.g., filaments, dense cores and extended cloud envelopes. In the ATOMS survey, we will mainly apply the principal component analysis (PCA; e.g., Lo et al. 2009; Jones et al. 2012, 2013) and histogram of oriented gradients (HOGs; e.g., Soler et al. 2019) analysis methods for this purpose. Below we will introduce the use of these two methods from the case study of G9.62+0.19.

\subsubsection{Principal component analysis}

The principal component analysis (PCA) is a statistical procedure to convert a set of observations of possibly correlated variables into a set of values of linearly uncorrelated variables called principal components. PCA has been widely used to identify and quantify similarities and differences between various molecular line emission inside molecular clouds (Lo et al. 2009; Jones et al. 2012, 2013; Spezzano et al. 2017; Nagy et al. 2019). We performed PCA on the integrated emission maps of various gas tracers to characterize their differences in spatial distribution in the G9.62+0.19 complex following a provider similar to that adopted in these previous works. The integrated intensity maps we used are presented in Figures A1, A2, and A3. These maps are firstly smoothed and re-gridded to ensure that they have the same resolution and pixel size. We then export their pixel values to SPSS software platform ${ }^{1}$ for PCA analysis. Table 5 lists the correlation matrix of the input molecules, the eigenvectors and the eigenvalues of the Principal Components. From the correlation matrix, one can see that the four dense-gas tracers $\left(\mathrm{CH}_{3} \mathrm{OH}, \mathrm{SO}, \mathrm{H}^{13} \mathrm{CN}\right.$ and $\left.\mathrm{HC}_{3} \mathrm{~N}\right)$ are strongly correlated with each other, with correlation coefficients above 0.8 . $\mathrm{CCH}$ shows strongest correlation with $\mathrm{H}^{13} \mathrm{CO}^{+}$with a correlation coefficient of 0.91 , indicating that these two molecules have very similar spatial distributions, as also mentioned in section 3.2.3. CS, $\mathrm{HCO}^{+}$and $\mathrm{HCN}$ are also strongly correlated with each other. This is consistent with the integrated intensity maps (Figure A1), showing that $\mathrm{CS}, \mathrm{HCO}^{+}$and $\mathrm{HCN}$ have similar large-scale spatial distribution (also see section 3.2.3). SiO is weakly correlated with most molecules except SO. The correlation coefficient between $\mathrm{SiO}$ and $\mathrm{SO}$ is $\sim 0.8$, suggesting that the two molecules trace similar shocked gas at large scale.

Figure 14 presents eigenvalues for each principal components in the PCA analysis. We identified three main principal components (or PCs 1-3) that have eigenvalues larger than 0.5. These three PCs

\footnotetext{
1 https://www.ibm.com/analytics/spss-statistics-software
} 

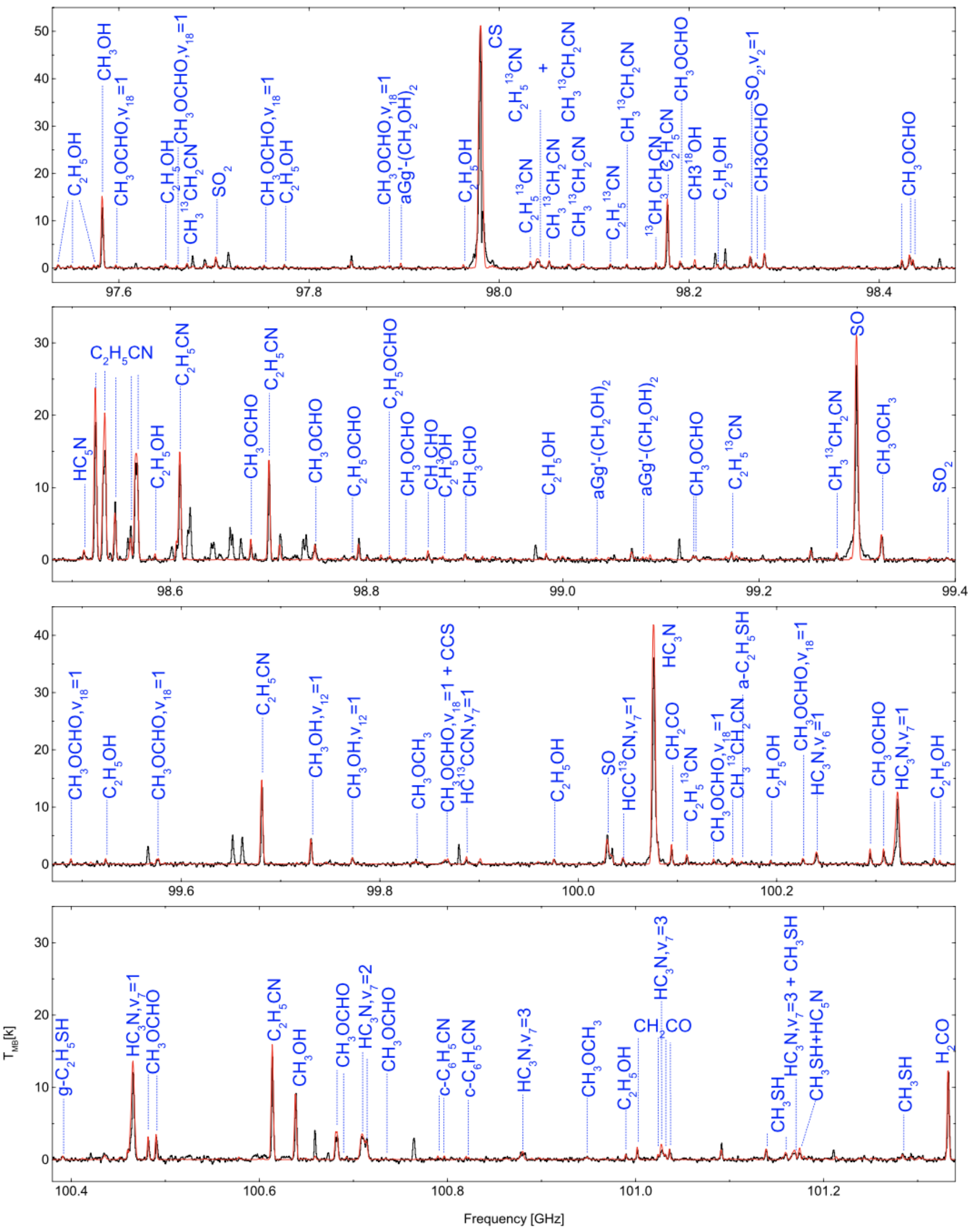

Figure 12. Sample spectra at the emission peak of G9.62+0.19 core MM8/F. The black curve is the observed spectra, and red curve indicates the simulated spectra with XCLASS. The identified molecular line transitions are labeled. 
(a) First wide spectral window

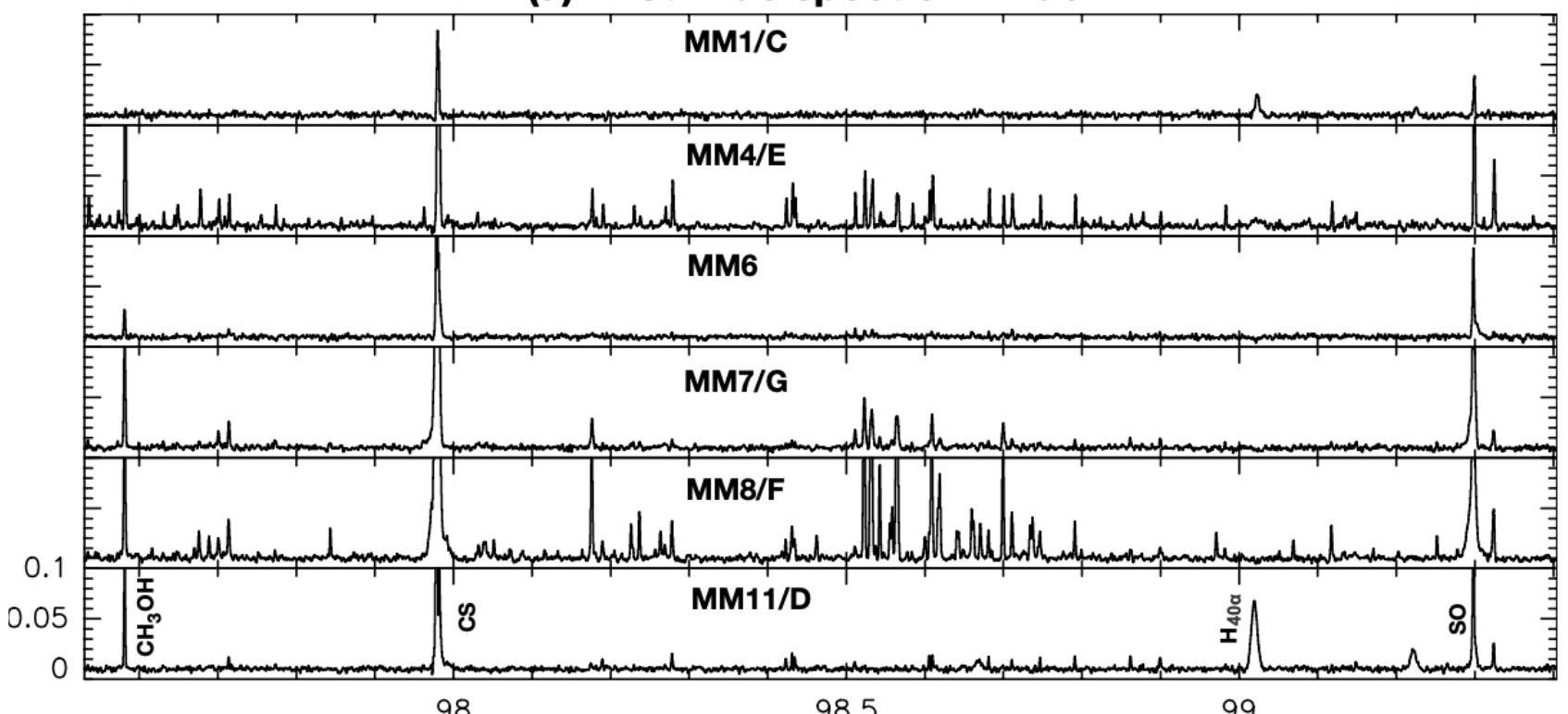

(b) Second wide spectral window

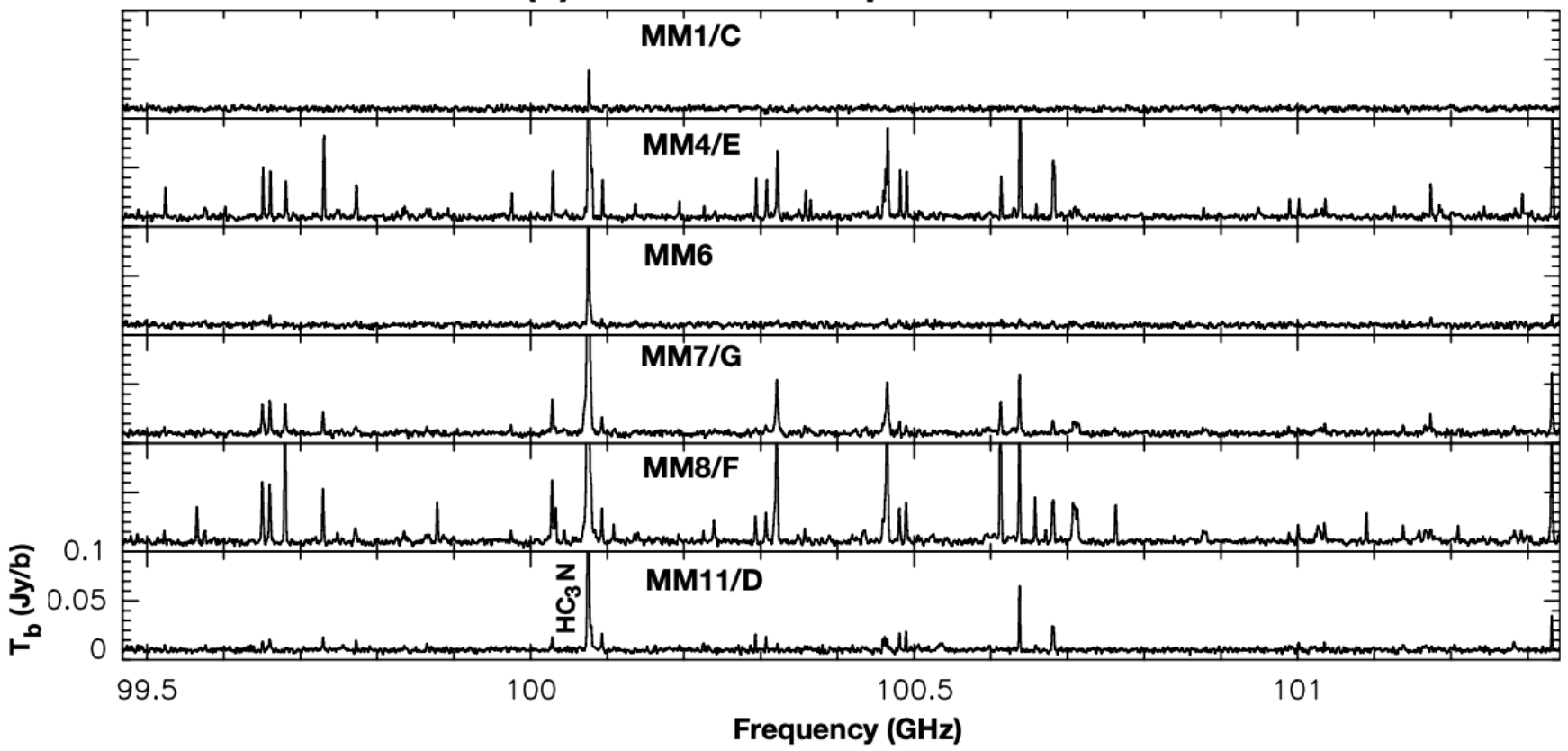

Figure 13. The line spectra of the two wide spectral window averaged over $2^{\prime \prime}$ at each core of G9.62+0.19.

describe $73.62,9.40$ and 6.22 per cent, respectively, of the variance in the data. They together account for nearly 90 per cent of total variation in the data set. The eigenvectors in PCA analysis describe correlations between the variable and the PCs. A (negative) positive eigenvector indicates the molecule is (anti-) correlated with the PC. The larger the value, the stronger the correlation. The eigenvectors of each molecule are listed in the last 10 rows in Table 5. Constructed images of the first three PCs are presented in Figure 15.

For the first PC (PC 1), all the ten molecules have large positive eigenvectors $(>0.7)$, suggesting that all ten molecules are posi- tively correlated with each other on large scales and PC 1 resembles the basis of molecular distribution within the G9.62+0.19 clump, as seen in Figure 15a. The PC 1 detects well the dense cores as well as the large-scale extended structures.

Figure 16 shows the plot of eigenvectors of each molecule in PC 2 and PC 3. From this plot, we roughly classify the 10 molecules into three groups as separated by the two mutually perpendicular dashed lines. The two dashed lines are carefully chosen to ensure that the angles between a pair of eigenvectors in each group are smaller than $90^{\circ}$. The first group includes $\mathrm{CS}, \mathrm{HCO}^{+}, \mathrm{HCN}, \mathrm{CCH}$ and $\mathrm{H}^{13} \mathrm{CO}^{+}$, which mainly trace extended gas emission and are 


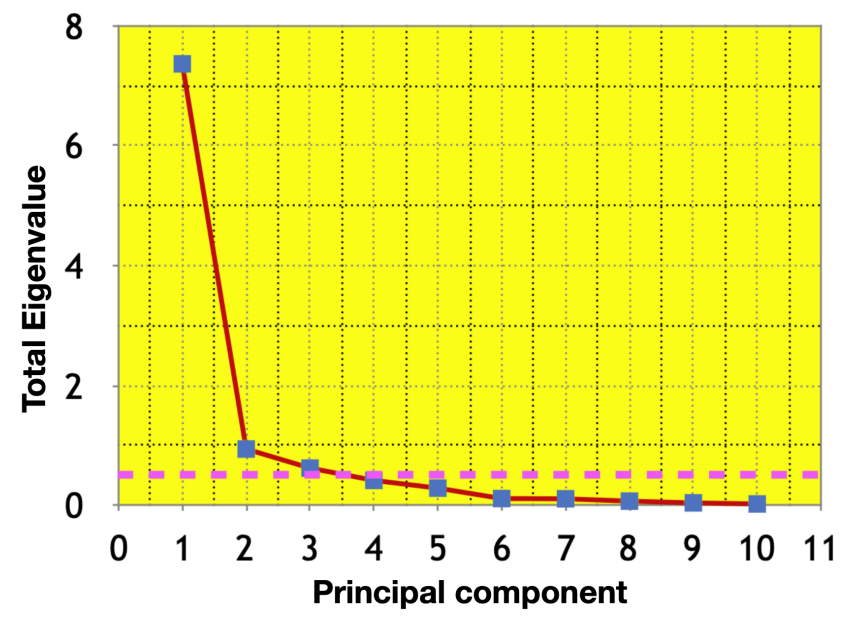

Figure 14. The eigenvalue against the component number of the 10 principle components (PCs). The dashed line marks an eigenvalue threshold of 0.5 .

poor tracers of dense cores. The third group includes $\mathrm{SO}, \mathrm{CH}_{3} \mathrm{OH}$, $\mathrm{H}^{13} \mathrm{CN}$ and $\mathrm{HC}_{3} \mathrm{~N}$, which are dense core tracers. $\mathrm{SiO}$ in the second group stands out from other molecules because it mainly traces shocked gas. The first and third groups are clearly anti-correlated in Figure 16. This division of groups, however, is not strict. For example, although $\mathrm{SO}$ and $\mathrm{SiO}$ are in different groups, the angle between their eigenvectors is only $48^{\circ}$, indicating that they have high similarities somehow. As discussed before, $\mathrm{SiO}$ is strongly correlated with SO because both lines are good shock tracers. SO is assigned to the third group because SO is also tracing dense cores as well as other tracers in the same group.

Different groups are also clearly distinguished in the constructed images of the PC 2 and PC 3 (see Figures 15b and 15c). The dense core emission and shocked gas emission are shown in negative contours in the constructed image of the PC 2, while the extended gas emission is shown in solid contours. From the eigenvector plot of the third PC (PC 3 axis of Figure 16), three molecules $\left(\mathrm{HCN}, \mathrm{H}^{13} \mathrm{CN}\right.$ and $\mathrm{CH}_{3} \mathrm{OH}$ ) show negative eigenvector smaller than -0.2. They mainly trace dense cores as shown in negative contours in Figure 15c. HCN emission is also strongly anticorrelated to PC 3 because its emission is centered on the dense core "MM4/E" in the constructed image of $\mathrm{PC} 3 . \mathrm{H}^{13} \mathrm{CO}^{+}$and $\mathrm{CCH}$ are strongly correlated with $\mathrm{PC} 3$ with eigenvector larger than $\sim 0.2$, while $\mathrm{CS}$ and $\mathrm{HCO}^{+}$are weakly correlated with $\mathrm{PC} 3$ with eigenvector smaller than $\sim 0.1$. It suggests that $\mathrm{H}^{13} \mathrm{CO}^{+}$and $\mathrm{CCH}$ may trace some gas emission structures that are not well traced by $\mathrm{CS}$ and $\mathrm{HCO}^{+}$(Figure 15c). Interestingly, we found that $\mathrm{H}^{13} \mathrm{CO}^{+}$ (also $\mathrm{CCH}$ ) is clearly anti-correlated with $\mathrm{H}^{13} \mathrm{CN}$ in the PC 2 and PC 3. The angle between their eigenvectors in Figure 16 is close to $180^{\circ}$, suggesting that they trace very different structures in these two PCs. It is because $\mathrm{H}^{13} \mathrm{CO}^{+}$and $\mathrm{CCH}$ do not trace the dense cores at all, while $\mathrm{H}^{13} \mathrm{CN}$ emission coincides with the dense core emission very well.

From $\mathrm{PCA}$ analysis, we learned that $\mathrm{CS}, \mathrm{HCO}^{+}, \mathrm{HCN}, \mathrm{CCH}$ and $\mathrm{H}^{13} \mathrm{CO}^{+}$show high similarities in spatial distribution because they mainly trace extended gas emission and they are poor tracers of dense cores in $\mathrm{G} 9.62+0.19$. In contrast, $\mathrm{CH}_{3} \mathrm{OH}, \mathrm{H}^{13} \mathrm{CN}$ and $\mathrm{HC}_{3} \mathrm{~N}$ trace well dense cores. $\mathrm{SiO}$ line is a pure shock tracer. It has high similarity with another shock tracer, SO. However, SO line emission can trace well not only shocked gas but also dense cores.
The PCA analysis is very powerful in separating different kinds of gas and will be used in further studies in the ATOMS survey to characterize gas distributions.

\subsubsection{Histogram of oriented gradients analysis.}

An alternative way to study the spatial correlation between different tracers in molecular clouds is the Histogram of Oriented Gradients analysis (HOGs; Soler et al. 2019). In the HOGs method, it is assumed that the appearance and shape of an object in an image can be well characterized by the distribution of local intensity gradients or edge directions. The HOG method quantifies similarities and differences in two images by studying the occurrences of the relative orientations between their local gradient orientations. Soler et al. (2019) investigated the spatial correlation of the $\mathrm{HI}$ and ${ }^{13} \mathrm{CO}$, and found a significant spatial correlation between the two tracers in velocity channels where $V_{\mathrm{HI}} \approx V^{13} \mathrm{CO}$.

In this work, we performed a HOGs analysis on the integrated intensity maps of various gas tracers following the procedure in Soler et al. (2019). Three statistical evaluation parameters are derived: mean resultant vector length $(r)$, the projected Rayleigh statistic $(V)$, and the alignment measurement $(A M)$. The definitions of these parameters are given in Soler et al. (2019). The resultant vector length, $r$, is a normalized quantity that can be interpreted as the fraction of uniform distribution of angles between gradient vectors. A preferential relative orientation (either parallel or perpendicular) in two images is of statistical significance if $r$ differs from zero significantly. The projected Rayleigh statistic $(V)$ is used to determine whether or not gradient vectors have mostly parallel $(V>0)$ or perpendicular $(V<0)$ relative orientations. The higher the $V$ values, the more similarity in two images. The alignment measurement $(A M)$, is an alternative method for estimating the degree of alignment between vectors (Lazarian \& Hoang 2007). High $V$ and $A M$ values indicate mostly parallel relative orientations between the gradient vectors.

Three statistical evaluation parameters from HOGs analysis are summarized in Table 6. The three parameters give consistent statistical evaluation results. $\mathrm{HC}_{3} \mathrm{~N}$ is strongly correlated with six molecules $\left(\mathrm{H}^{13} \mathrm{CN}, \mathrm{H}^{13} \mathrm{CO}^{+}, \mathrm{HCO}^{+}, \mathrm{CCH}, \mathrm{SO}, \mathrm{CS}\right)$, with $r>0.2$, $V>1000$ and $A M>0.4$. This correlation arises because $\mathrm{HC}_{3} \mathrm{~N}$ is a good tracer for both dense cores and extended structures (see Figure A3). The strongest correlation occurs between $\mathrm{HC}_{3} \mathrm{~N}$ and $\mathrm{H}^{13} \mathrm{CN}$ with $r$ of $0.43, V$ of 1770 and $A M$ of 0.61 . These two molecules have similar geometry in their integrated intensity maps (see Figure A3). $\mathrm{H}^{13} \mathrm{CO}^{+}$is most correlated with $\mathrm{CCH}$, as also mentioned in section 3.2.3. The $r, V$ and $A M$ values for the pair of $\mathrm{H}^{13} \mathrm{CO}^{+}$and $\mathrm{CCH}$ are $0.33,2051$ and 0.54 , respectively. CS shows highest correlation with $\mathrm{HCO}^{+}$with $V$ of 2373 and $A M$ of 0.43 , suggesting that they have very similar spatial distributions. $\mathrm{HCN}$ shows strongest correlations with $\mathrm{CS}$ and $\mathrm{HCO}^{+}$with $V>800$ and $A M>0.3$. The $\mathrm{SiO}$ image is most similar to SO image with $V$ of 1078 and $A M$ of 0.54, suggesting that $\mathrm{SiO}$ and $\mathrm{SO}$ may reveal similar shocked gas distribution. $\mathrm{CH}_{3} \mathrm{OH}$ shows strongest correlations $(V \gtrsim 450$ and $A M \gtrsim 0.45)$ with $\mathrm{H}^{13} \mathrm{CN}, \mathrm{HC}_{3} \mathrm{~N}$ and $\mathrm{SO}$ because they trace similar dense gas distribution in the massive filament.

The HOGs analysis is also consistent with the previous PCA analysis. Molecules in the same group defined by the PCA analysis in Figure 14 show the strongest correlations in the HOGs analysis.

We also investigate the similarities and differences between the $3 \mathrm{~mm}$ continuum emission and gas emission. We only performed HOGs analysis in the massive filament region. For example, 


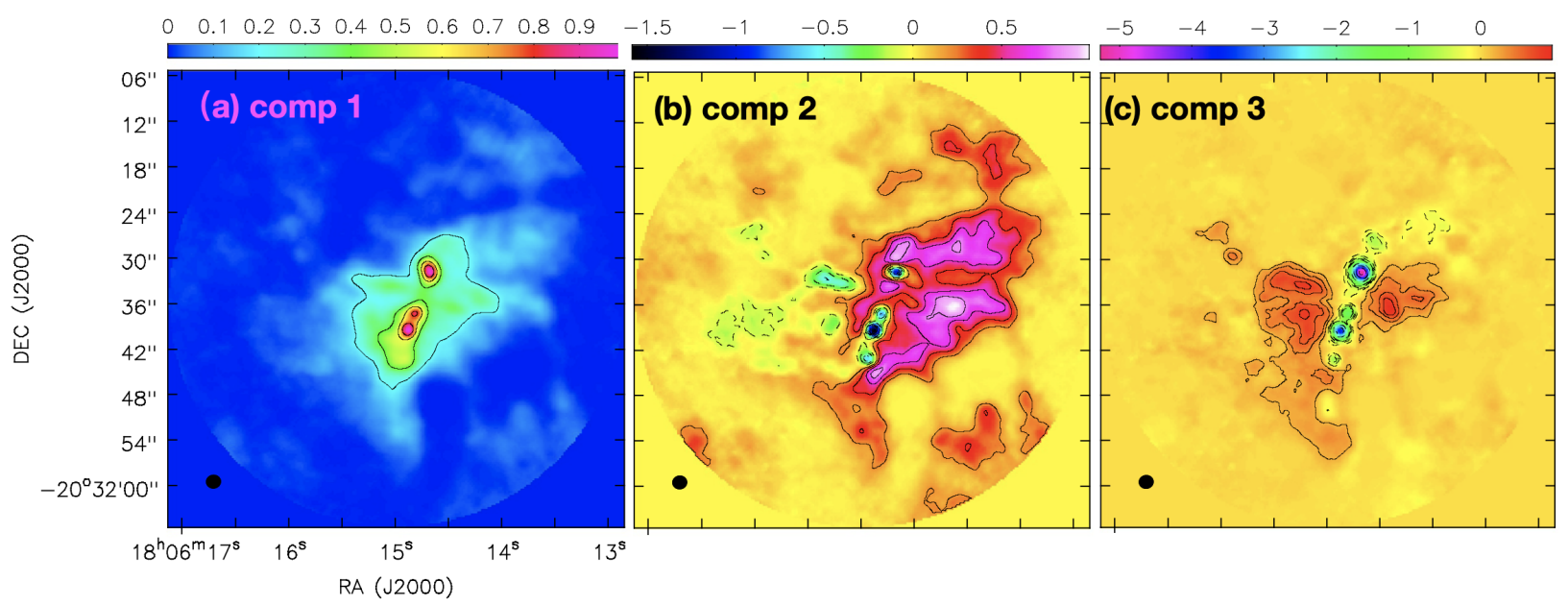

Figure 15. Constructed images of the first three PCs. The images are normalized to their maximum values. (a) Principal Component 1 . The contours are 0.2,0.4,0.6,0.8 (b) Principal Component 2. The contours are -1.5,-1.3,-1.1,-0.1,0.2,0.4,0.6,0.8 (c) Principal Component 3. The contours are -5,-4,-3,-2,-1,-0.8,$0.6,-0.4,-0.2,0.2,0.4,0.6,0.8$.

Table 5. PCA analysis of G9.62+0.19.

\begin{tabular}{|c|c|c|c|c|c|c|c|c|c|c|}
\hline \multicolumn{11}{|c|}{ The correlation matrix of the input molecular dataset } \\
\hline & $\mathrm{CCH}$ & $\mathrm{CH}_{3} \mathrm{OH}$ & CS & $\mathrm{H}^{13} \mathrm{CN}$ & $\mathrm{H}^{13} \mathrm{CO}^{+}$ & $\mathrm{HC}_{3} \mathrm{~N}$ & $\mathrm{HCN}$ & $\mathrm{HCO}^{+}$ & $\mathrm{SiO}$ & SO \\
\hline $\mathrm{CCH}$ & 1.00 & 0.56 & 0.79 & 0.70 & 0.91 & 0.77 & 0.59 & 0.73 & 0.60 & 0.75 \\
\hline $\mathrm{CH}_{3} \mathrm{OH}$ & 0.56 & 1.00 & 0.57 & 0.83 & 0.62 & 0.87 & 0.48 & 0.47 & 0.56 & 0.84 \\
\hline $\mathrm{CS}$ & 0.79 & 0.57 & 1.00 & 0.81 & 0.76 & 0.75 & 0.68 & 0.87 & 0.57 & 0.81 \\
\hline $\mathrm{H}^{13} \mathrm{CN}$ & 0.70 & 0.83 & 0.81 & 1.00 & 0.75 & 0.94 & 0.63 & 0.70 & 0.54 & 0.87 \\
\hline $\mathrm{H}^{13} \mathrm{CO}^{+}$ & 0.91 & 0.62 & 0.76 & 0.75 & 1.00 & 0.83 & 0.62 & 0.75 & 0.60 & 0.80 \\
\hline $\mathrm{HC}_{3} \mathrm{~N}$ & 0.77 & 0.87 & 0.75 & 0.94 & 0.83 & 1.00 & 0.62 & 0.69 & 0.66 & 0.92 \\
\hline $\mathrm{HCN}$ & 0.59 & 0.48 & 0.68 & 0.63 & 0.62 & 0.62 & 1.00 & 0.79 & 0.35 & 0.60 \\
\hline $\mathrm{HCO}^{+}$ & 0.73 & 0.47 & 0.87 & 0.70 & 0.75 & 0.69 & 0.79 & 1.00 & 0.48 & 0.71 \\
\hline $\mathrm{SiO}$ & 0.60 & 0.56 & 0.57 & 0.54 & 0.60 & 0.66 & 0.35 & 0.48 & 1.00 & 0.78 \\
\hline $\mathrm{SO}$ & 0.75 & 0.84 & 0.81 & 0.87 & 0.80 & 0.92 & 0.60 & 0.71 & 0.78 & 1.00 \\
\hline \multicolumn{11}{|c|}{ The eigenvectors and eigenvalues of the Principle Components } \\
\hline Component & 1 & 2 & 3 & 4 & 5 & 6 & 7 & 8 & 9 & 10 \\
\hline Percentage of variance $(\%)$ & 73.62 & 9.40 & 6.22 & 4.18 & 2.89 & 1.16 & 1.12 & 0.72 & 0.43 & 0.26 \\
\hline $\mathrm{CCH}$ & 0.87 & 0.13 & 0.27 & -0.32 & 0.09 & 0.13 & 0.10 & 0.10 & -0.06 & 0.02 \\
\hline $\mathrm{CH}_{3} \mathrm{OH}$ & 0.80 & -0.45 & -0.34 & 0.00 & 0.07 & -0.02 & 0.20 & 0.01 & 0.07 & 0.01 \\
\hline $\mathrm{CS}$ & 0.89 & 0.25 & 0.06 & 0.05 & -0.33 & 0.14 & 0.05 & -0.08 & 0.06 & -0.05 \\
\hline $\mathrm{H}^{13} \mathrm{CN}$ & 0.91 & -0.12 & -0.28 & -0.07 & -0.16 & 0.05 & -0.18 & 0.07 & 0.03 & 0.08 \\
\hline $\mathrm{H}^{13} \mathrm{CO}^{+}$ & 0.90 & 0.08 & 0.18 & -0.30 & 0.16 & -0.11 & -0.07 & -0.13 & 0.08 & 0.01 \\
\hline $\mathrm{HC}_{3} \mathrm{~N}$ & 0.94 & -0.21 & -0.14 & -0.08 & 0.04 & -0.04 & -0.10 & 0.06 & -0.05 & -0.12 \\
\hline $\mathrm{HCN}$ & 0.74 & 0.47 & -0.25 & 0.26 & 0.31 & 0.10 & -0.02 & -0.02 & 0.00 & 0.00 \\
\hline $\mathrm{HCO}^{+}$ & 0.84 & 0.44 & 0.03 & 0.13 & -0.12 & -0.23 & 0.06 & 0.09 & -0.01 & 0.01 \\
\hline $\mathrm{SiO}$ & 0.71 & -0.36 & 0.48 & 0.35 & 0.07 & 0.02 & -0.05 & 0.05 & 0.05 & 0.01 \\
\hline SO & 0.95 & -0.22 & 0.02 & 0.10 & -0.05 & -0.03 & 0.03 & -0.14 & -0.14 & 0.04 \\
\hline
\end{tabular}

the gray image in the upper panel of Figure 17 shows the absolute values of relative orientation angles ( $\phi$; color image) between the $3 \mathrm{~mm}$ continuum map (yellow contours) and the $\mathrm{HC}_{3} \mathrm{~N}$ map (red contours) in the filament region. The histograms of relative orientation angles between $3 \mathrm{~mm}$ continuum and molecules are shown in the lower panel of Figure 17 and Figure 18. From the histograms, one can see that the distributions of relative orientation angles for $\mathrm{HC}_{3} \mathrm{~N}, \mathrm{H}^{13} \mathrm{CN}, \mathrm{CH}_{3} \mathrm{OH}$ and $\mathrm{SO}$ are clearly peaked around $0^{\circ}$ with small dispersions. However, the other molecules show much more flat distributions. It indicates that $\mathrm{HC}_{3} \mathrm{~N}, \mathrm{H}^{13} \mathrm{CN}, \mathrm{CH}_{3} \mathrm{OH}$ and $\mathrm{SO}$ are the best to trace the dense structures (filaments and cores) as the $3 \mathrm{~mm}$ continuum emission. The other molecules are poor tracers for those dense structures. As shown in Table $6, \mathrm{HC}_{3} \mathrm{~N}$ shows highest similarity with the $3 \mathrm{~mm}$ continuum emission. The $r, V$ and $A M$ for the $\mathrm{HC}_{3} \mathrm{~N}$ and $3 \mathrm{~mm}$ continuum correlation are $0.32,1138$ and 0.53 , respectively. $\mathrm{SiO}$ shows largest difference with the $3 \mathrm{~mm}$ continuum emission. The $r, V$ and $A M$ for the $\mathrm{SiO}$ and $3 \mathrm{~mm}$ continuum correlation are $0.08,119$ and 0.32 , respectively, indicating that $\mathrm{SiO}$ cannot trace dense structures at all. 
Table 6. HOG analysis of G9.62+0.19

\begin{tabular}{|c|c|c|c|c|c|c|c|c|c|c|c|}
\hline lines & continuum & $\mathrm{HC}_{3} \mathrm{~N}$ & $\mathrm{H}^{13} \mathrm{CN}$ & $\mathrm{H}^{13} \mathrm{CO}^{+}$ & $\mathrm{CCH}$ & CS & $\mathrm{HCO}^{+}$ & $\mathrm{HCN}$ & SO & $\mathrm{CH} 3 \mathrm{OH}$ & $\mathrm{SiO}$ \\
\hline \multicolumn{12}{|c|}{$r$} \\
\hline continuum & 1.00 & & & & & & & & & & \\
\hline $\mathrm{HC}_{3} \mathrm{~N}$ & 0.32 & 1.00 & & & & & & & & & \\
\hline $\mathrm{H}^{13} \mathrm{CN}$ & 0.30 & 0.43 & 1.00 & & & & & & & & \\
\hline $\mathrm{H}^{13} \mathrm{CO}^{+}$ & 0.10 & 0.33 & 0.16 & 1.00 & & & & & & & \\
\hline $\mathrm{CCH}$ & 0.13 & 0.28 & 0.18 & 0.33 & 1.00 & & & & & & \\
\hline $\mathrm{CS}$ & 0.11 & 0.20 & 0.29 & 0.08 & 0.16 & 1.00 & & & & & \\
\hline $\mathrm{HCO}^{+}$ & 0.11 & 0.17 & 0.21 & 0.18 & 0.12 & 0.19 & 1.00 & & & & \\
\hline $\mathrm{HCN}$ & 0.07 & 0.05 & 0.11 & 0.03 & 0.03 & 0.08 & 0.07 & 1.00 & & & \\
\hline SO & 0.20 & 0.29 & 0.29 & 0.12 & 0.14 & 0.19 & 0.09 & 0.09 & 1.00 & & \\
\hline $\mathrm{CH} 3 \mathrm{OH}$ & 0.24 & 0.26 & 0.25 & 0.02 & 0.03 & 0.10 & 0.05 & 0.08 & 0.23 & 1.00 & \\
\hline $\mathrm{SiO}$ & 0.08 & 0.15 & 0.06 & 0.12 & 0.07 & 0.05 & 0.07 & 0.04 & 0.33 & 0.21 & 1.00 \\
\hline \multicolumn{12}{|c|}{ V } \\
\hline continuum & 5278 & & & & & & & & & & \\
\hline $\mathrm{HC}_{3} \mathrm{~N}$ & 1138 & 6972 & & & & & & & & & \\
\hline $\mathrm{H}^{13} \mathrm{CN}$ & 1040 & 1770 & 4707 & & & & & & & & \\
\hline $\mathrm{H}^{13} \mathrm{CO}^{+}$ & 388 & 1961 & 712 & 8011 & & & & & & & \\
\hline $\mathrm{CCH}$ & 512 & 1714 & 774 & 2051 & 9193 & & & & & & \\
\hline $\mathrm{CS}$ & 571 & 1334 & 1387 & 546 & 1267 & 15850 & & & & & \\
\hline $\mathrm{HCO}^{+}$ & 485 & 1083 & 964 & 1278 & 972 & 2373 & 18196 & & & & \\
\hline $\mathrm{HCN}$ & 317 & 300 & 503 & -24 & 179 & 809 & 845 & 16265 & & & \\
\hline SO & 823 & 1620 & 1243 & 764 & 701 & 1514 & 598 & 554 & 8115 & & \\
\hline $\mathrm{CH} 3 \mathrm{OH}$ & 391 & 644 & 448 & 28 & 57 & 266 & 90 & 179 & 661 & 2933 & \\
\hline $\mathrm{SiO}$ & 119 & 381 & 119 & 163 & 138 & 169 & 169 & 156 & 1078 & 452 & 3354 \\
\hline \multicolumn{12}{|c|}{$A M$} \\
\hline continuum & 1.00 & & & & & & & & & & \\
\hline $\mathrm{HC}_{3} \mathrm{~N}$ & 0.53 & 1.00 & & & & & & & & & \\
\hline $\mathrm{H}^{13} \mathrm{CN}$ & 0.51 & 0.61 & 1.00 & & & & & & & & \\
\hline $\mathrm{H}^{13} \mathrm{CO}^{+}$ & 0.35 & 0.54 & 0.41 & 1.00 & & & & & & & \\
\hline $\mathrm{CCH}$ & 0.38 & 0.50 & 0.42 & 0.54 & 1.00 & & & & & & \\
\hline $\mathrm{CS}$ & 0.37 & 0.43 & 0.51 & 0.33 & 0.40 & 1.00 & & & & & \\
\hline $\mathrm{HCO}^{+}$ & 0.36 & 0.42 & 0.44 & 0.42 & 0.37 & 0.43 & 1.00 & & & & \\
\hline $\mathrm{HCN}$ & 0.33 & 0.32 & 0.37 & 0.27 & 0.29 & 0.33 & 0.32 & 1.00 & & & \\
\hline SO & 0.44 & 0.50 & 0.50 & 0.37 & 0.38 & 0.43 & 0.34 & 0.35 & 1.00 & & \\
\hline $\mathrm{CH} 3 \mathrm{OH}$ & 0.46 & 0.46 & 0.46 & 0.29 & 0.30 & 0.35 & 0.31 & 0.34 & 0.45 & 1.00 & \\
\hline $\mathrm{SiO}$ & 0.32 & 0.38 & 0.33 & 0.33 & 0.31 & 0.32 & 0.32 & 0.30 & 0.54 & 0.44 & 1.00 \\
\hline
\end{tabular}

\subsection{Fraction of line fluxes from filaments and cores}

One of the main science goals of the ATOMS survey is to statistically evaluate what portion of gas in the gravitationally bound clumps is actually participating in star formation or is concentrated in the smallest star formation units, i.e., dense cores. In this section, we explore what fraction of the fluxes of the various lines comes from the massive filament that is forming high-mass stars in G9.62+0.19.

In Table 7, we compare the total integrated intensities of various molecular lines obtained from ACA measurements with that obtained from 12-m array measurements. The integrated intensities are derived in the velocity interval of 1.5 to $6.5 \mathrm{~km} \mathrm{~s}^{-1}$. The deconvolved FWHM angular sizes, peak integrated intensities and total integrated intensities $\left(S_{v}^{\mathrm{ACA}}\right)$ of various molecular lines in ACA data are derived from a 2D gaussian fitting, and are listed in columns 2-4 in Table 7. The total integrated intensities $\left(\mathrm{S}_{v}^{12 \mathrm{~m}}\right)$ of molecular lines in 12-m array data are measured within the massive filament region that is bounded by the $1 \%$ of peak flux density contour ( $\sim 3.5 \sigma$ level) of the $3 \mathrm{~mm}$ continuum emission. We did not perform 2D Gaussian fitting on 12-m array data because the massive filament is far from a 2D Gaussian shape. We also did not measure integrated intensities of molecular lines in the HII region " $\mathrm{B}$ " because there is no molecular line emission associated with it. The $1 \%$ of peak flux density contour of the $3 \mathrm{~mm}$ continuum emission defines well the boundary of the massive filament (see Figure 5). The ratios of $S_{v}^{12 \mathrm{~m}}$ to $S_{v}^{\mathrm{ACA}}$ listed in the last column in Table 7 can be used to evaluate how much emission is from the densest filament region. Figure 19 shows the ratios against the $A M$ values that are derived from HOGs analysis. There is a clear trend between the ratios and the $A M$ values. Molecules with higher $A M$ values show higher similarity with the $3 \mathrm{~mm}$ continuum emission. Molecules $\left(\mathrm{CH}_{3} \mathrm{OH}, \mathrm{HC}_{3} \mathrm{~N}\right.$ and $\left.\mathrm{H}^{13} \mathrm{CN}\right)$ that have high $A M$ values also show high ratios of $S_{v}^{12 \mathrm{~m}}$ to $S_{v}^{\mathrm{ACA}}(>40 \%)$, further suggesting that these molecular lines are good tracers of the dense filament. In contrast, only less than $25 \%$ of the fluxes of $\mathrm{CCH}, \mathrm{HCN}, \mathrm{CS}$ and $\mathrm{HCO}^{+}$are from the dense filament region. These molecules also show largest differences (small AM values) with the spatial distribution of the 3 mm continuum emission.

G9.62+0.19 is a gravitationally bound massive clump (Liu et al. 2017, 2018c). Molecular lines HCN J=1-0, CS J=2-1 and $\mathrm{HCO}^{+}$ 
Table 7. Integrated intensities of molecular lines in ACA and 12-m array observations.

\begin{tabular}{|c|c|c|c|c|c|}
\hline \multirow[t]{2}{*}{ Molecule } & \multicolumn{3}{|c|}{$\mathrm{ACA}^{a}$} & \multirow{2}{*}{$\begin{array}{c}12-\mathrm{m}^{b} \\
\text { total } \\
\left(\mathrm{Jy} \mathrm{km} \mathrm{s}^{-1}\right)\end{array}$} & \multirow[b]{2}{*}{ ratio } \\
\hline & $\begin{array}{l}\text { Size } \\
\left({ }^{\prime \prime},{ }^{\prime \prime}\right)\end{array}$ & $\begin{array}{c}\text { peak } \\
\left(\mathrm{Jy} \mathrm{beam}^{-1} \mathrm{~km} \mathrm{~s}^{-1}\right)\end{array}$ & $\begin{array}{c}\text { total } \\
\left(\mathrm{Jy} \mathrm{km} \mathrm{s}^{-1}\right)\end{array}$ & & \\
\hline $\mathrm{H}^{13} \mathrm{CN}$ & $17.6^{\prime \prime} \times 11.4^{\prime \prime}\left(149^{\circ}\right)$ & 15.3 & 32.3 & 13.5 & 0.42 \\
\hline $\mathrm{H}^{13} \mathrm{CO}^{+}$ & $24.0^{\prime \prime} \times 12.5^{\prime \prime}\left(127^{\circ}\right)$ & 15.0 & 38.1 & 11.2 & 0.29 \\
\hline $\mathrm{CCH}$ & $26.2^{\prime \prime} \times 13.5^{\prime \prime}\left(142^{\circ}\right)$ & 13.0 & 38.7 & 8.7 & 0.23 \\
\hline $\mathrm{SiO}$ & $18.1^{\prime \prime} \times 11.4^{\prime \prime}\left(93^{\circ}\right)$ & 10.3 & 20.7 & 4.6 & 0.22 \\
\hline $\mathrm{HCN}$ & $34.3^{\prime \prime} \times 22.0^{\prime \prime}\left(131^{\circ}\right)$ & 12.1 & 46.4 & 8.5 & 0.18 \\
\hline $\mathrm{HCO}^{+}$ & $31.4^{\prime \prime} \times 15.7^{\prime \prime}\left(137^{\circ}\right)$ & 23.6 & 89.3 & 15.3 & 0.17 \\
\hline $\mathrm{CH}_{3} \mathrm{OH}$ & $13.7^{\prime \prime} \times 7.0^{\prime \prime}\left(122^{\circ}\right)$ & 10.6 & 17.2 & 10.1 & 0.59 \\
\hline CS & $23.5^{\prime \prime} \times 13.7^{\prime \prime}\left(135^{\circ}\right)$ & 53.9 & 166.3 & 38.1 & 0.23 \\
\hline SO & $17.2^{\prime \prime} \times 10.9^{\prime \prime}\left(119^{\circ}\right)$ & 28.8 & 62.2 & 19.4 & 0.31 \\
\hline $\mathrm{HC}_{3} \mathrm{~N}$ & $15.4^{\prime \prime} \times 8.9^{\prime \prime}\left(140^{\circ}\right)$ & 34.5 & 70.2 & 35.9 & 0.51 \\
\hline
\end{tabular}

${ }^{a}$ The parameters from ACA data were from 2D Gaussian fits.

${ }^{b}$ The total integrated intensities of molecular lines in $12-\mathrm{m}$ array data are measured within the massive filament region bounded by the $1 \%$ of peak flux density contour ( $\sim 3.5 \sigma$ level) of the $3 \mathrm{~mm}$ continuum emission.

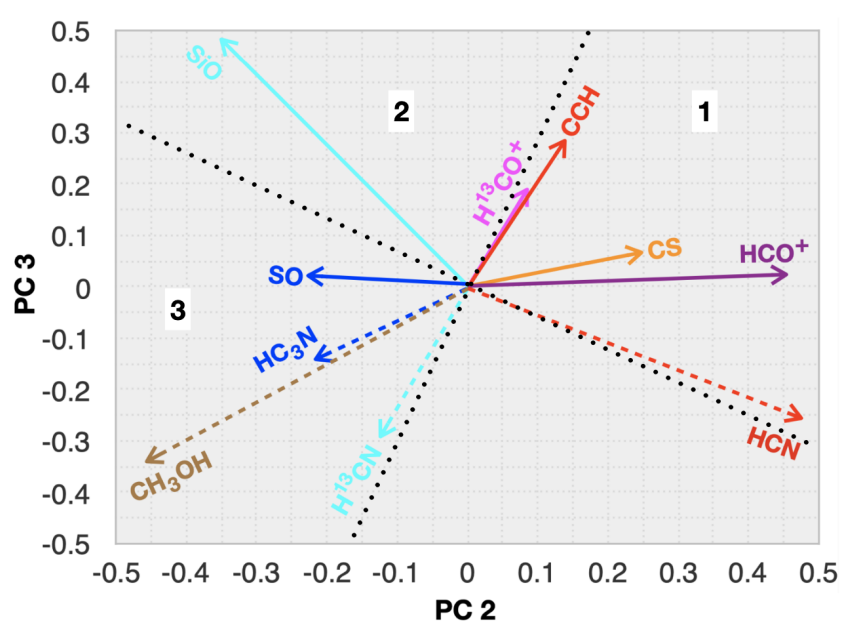

Figure 16. Plots of eigenvectors of the second and third PCs of the data set. Each of the eigenvectors represents the component of that molecule in the relevant PC. The two mutually perpendicular dashed lines separate the molecules into three groups.

$\mathrm{J}=1-0$ are commonly used in dense gas star formation law studies (Gao \& Solomon 2004; Wu et al. 2005, 2010). Our studies indicate that even in such a gravitationally bound clump, these commonly used lines are poor tracers of the dense structures, e.g., filament or dense cores. This is consistent with observations in nearby GMCs, where these lines do not well trace the column density structures (cores and filaments) revealed in Herschel images (Pety et al. 2017; Shimajiri et al. 2017). Our studies of G9.62+0.19 indicate that the gas lies within the dense filament as defined by the continuum emission only contributes to less than $25 \%$ flux of these lines. These main lines of dense gas tracers are poor tracers of dense structures because they tend to be optically thick in dense parts of molecular clouds (e.g., Sanhueza et al. 2012; Hoq et al. 2013; Shimajiri et al. 2017; Liu et al. 2020). The large optical depths of main lines, such as $\mathrm{HCN}$ and $\mathrm{HCO}^{+}$, indicate that the density or mass estimates from their line intensity could be misleading. In contrast to the main lines, emission from the isotopologues are optically thinner because of their much lower abundances and hence higher effective excitation densities (Shirley 2015). Therefore, they are potentially better tracers of the column densities and dense structures in molecular clouds. The optical depth issues in dense gas star formation law studies with main lines will be discussed in a companion paper by Liu et al. (2020).

In dense gas star formation law studies (e.g., Wu et al. 2010), the line luminosity $\left(\mathrm{L}_{\mathrm{mol}}^{\prime}\right)$ is assumed as a tracer for dense gas mass $\left(\mathrm{M}_{\text {dense }}\right)$. Wu et al. (2010) found that the median conversion factors from line luminosity to dense gas masses $\left(\mathrm{L}_{\mathrm{mol}}^{\prime}\right.$-to- $\left.\mathrm{M}_{\text {dense }}\right)$ are about $20,39,16$ and $19 \mathrm{M}_{\odot}\left(\mathrm{K} \mathrm{km}^{-1} \mathrm{pc}^{2}\right)^{-1}$ for CS J=2-1, CS J=7$6, \mathrm{HCN} \mathrm{J}=1-0$ and $\mathrm{HCN} \mathrm{J}=3-2$ lines, respectively. In the ATOMS survey, we are more interested in how the total line luminosity of clumps correlates to the total masses $\left(\mathrm{M}_{\text {cores }}\right)$ of dense cores, i.e., the smallest star formation units. In G9.62+0.19, the total line flux densities of $\mathrm{H}^{13} \mathrm{CN}, \mathrm{H}^{13} \mathrm{CO}^{+}, \mathrm{HCN}$ and $\mathrm{HCO}^{+}$that are integrated over all velocity channels in ACA observations are 87, 45, 258 and $176 \mathrm{~K} \mathrm{~km}^{-1}$, respectively. We can convert the total flux densities of these lines into their line luminosity following Solomon et al. (1997). The line luminosity of the $\mathrm{J}=1-0$ transitions of $\mathrm{H}^{13} \mathrm{CN}$, $\mathrm{H}^{13} \mathrm{CO}^{+}, \mathrm{HCN}$ and $\mathrm{HCO}^{+}$of the G9.62+0.19 clump are 10, 5, 29 and $19 \mathrm{~K} \mathrm{~km}^{-1} \mathrm{pc}^{2}$, respectively. Liu et al. (2017) identified 12 dense cores in G9.62+0.19. The total mass of these dense cores is $\sim 400 \mathrm{M}_{\odot}$ assuming an average dust temperature of $35 \mathrm{~K}$ (Liu et al. 2017). Therefore, the conversion factors $\mathrm{L}_{\text {mol }}^{\prime}$-to- $\mathrm{M}_{\text {cores }}$ in G9.62+0.19 are about 40, 80, 14, and $21 \mathrm{M}_{\odot}\left(\mathrm{K} \mathrm{km}^{-1} \mathrm{pc}^{2}\right)^{-1}$ for the $\mathrm{J}=1-0$ transitions of $\mathrm{H}^{13} \mathrm{CN}, \mathrm{H}^{13} \mathrm{CO}^{+}, \mathrm{HCN}$ and $\mathrm{HCO}^{+}$, respectively. In future, we will statistically investigate the conversion factors of line luminosity to dense core masses with the full ATOMS sample, which is very helpful to investigate how star formation efficiency varies across Galactic clumps. The knowledge of these studies can also be applied to star formation in external galaxies.

\section{DISCUSSION}

\subsection{Stellar feedback and sequential high-mass star formation}

The ATOMS aims to investigate how stellar feedback from formed $\mathrm{OB}$ (proto-)stars in proto-clusters affect their natal clumps and reg- 

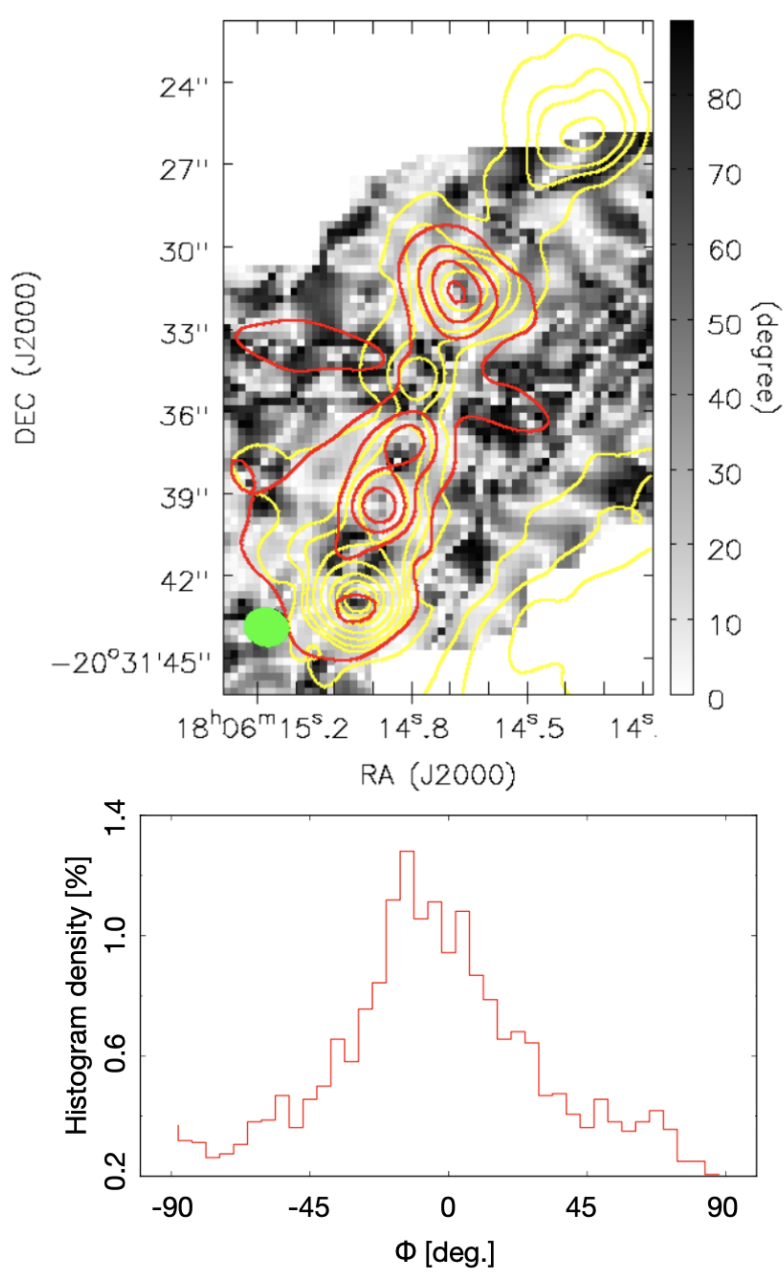

Figure 17. Upper panel: The background image shows the absolute values of relative orientation angles $(\phi)$ between the $3 \mathrm{~mm}$ continuum image and the $\mathrm{HC}_{3} \mathrm{~N}$ image of $\mathrm{G} 9.62+0.19$. The yellow contours show the $3 \mathrm{~mm}$ continuum emission. The contour levels are $[0.01,0.03,0.05,0.07,0.1,0.2,0.4,0.6,0.8] \times 74.1 \mathrm{mJy} / \mathrm{beam}$. The $\mathrm{HC}_{3} \mathrm{~N}$ integrated intensity map is shown in red contours. The contour levels are $[0.2,0.4,0.6,0.8] \times 3.09 \mathrm{Jy} / \mathrm{beam} \mathrm{km} / \mathrm{s}$. Lower panel: Histogram of relative orientation angles.

ulate new star formation. Below we discuss the stellar feedback effect in G9.62+0.19.

As discussed in section 3.2.8 and in Liu et al. (2017), sequential high-mass star formation is taking place inside the G9.62+0.19 clump, which has a size of $\sim 1 \mathrm{pc}$. The oldest HII regions in this area seem to have great influence on their natal clump, creating a cavity in molecular line emission. From moment 1 maps (see middle panels of Figure A2 and A3) of several molecular lines (e.g., $\mathrm{CCH}, \mathrm{H}^{13} \mathrm{CO}^{+}$, SO and $\mathrm{HC}_{3} \mathrm{~N}$ ), one can see clearly a velocity gradient across the clump. Such large-scale velocity gradient and widespread low-velocity $\mathrm{SiO}$ shocks surrounding the HII regions are more likely caused by the expansion of the HII region "B". It indicates that stellar feedback from formed OB proto-stars can greatly reshape their natal clumps and change the spatial distribution of gas.

We also noticed that the evolved OB proto-stars in G9.62+0.19 are not located near the center of the clump but are clearly close to the clump edges. Along the massive filament, most evolved sources are located at the ends and the youngest sources are located in the filament center. This phenomenon is similar to the "edgecollapse" process proposed for filaments (Pon et al. 2011, 2012; Clarke \& Whitworth 2015). For long filaments with aspect ratios larger than 2, the global collapse of the filaments is likely enddominated (Clarke \& Whitworth 2015). The ends experience the highest acceleration, and this leads to the formation of end-clumps which then converge on the centre. In addition, recent ALMA highresolution observations of massive infrared dark clouds (IRDCs) indicate that there is no significant evidence of primordial mass segregation (Contreras et al. 2018; Sanhueza et al. 2019). More massive cores are distributed in the same way as other low-mass cores (Contreras et al. 2018; Sanhueza et al. 2019). The observations of those IRDCs are consistent with what we have found here in the G9.62+0.19 clump. It seems that high-mass stars are not necessarily formed at the clump center as expected by the "competitive accretion" model (Bonnell et al. 2002; Bonnell \& Bate 2006; Bonnel 2008). Recently, Padoan et al. (2019) proposed a new scenario of massive-star formation, the "Inertial-Inflow Model", in which massive stars are assembled by large-scale, converging, inertial flows that naturally occur in supersonic turbulence.

Based on our knowledge of the G9.62+0.19 clump and recent studies of IRDCs, we propose the following scenario to explain the sequential high-mass star formation in pc-size clumps. The sketch of the scenario is shown in Figure 20. The three stages involved in proto-cluster formation of this scenario are:

(a) The initial fragmentation of a massive clump does not show significant evidence of primordial mass segregation (Sanhueza et al. 2019). The clumps are accreting gas from large-scale converging flows (Padoan et al. 2019). These flows generate low-velocity shocks that excite widespread narrow $\mathrm{SiO}$ emission.

(b) As proposed in the "edge-collapse" scenario (Pon et al. 2011, 2012; Clarke \& Whitworth 2015), dense cores that are near the clump edges or at the ends of dense filaments inside clumps have higher accretion rates and are more gravitationally unstable. They will be the first to collapse to form proto-stars. Dense cores and formed proto-stars keep accreting gas from clump gas. Highmass proto-stars could form away from clump center as seen in the G9.62+0.19 complex.

(c) Stellar feedback from formed OB stars reshapes their natal clumps and changes the spatial distribution of gas and dense cores. The expansion of HII regions can also generate low-velocity shocks that excite widespread narrow $\mathrm{SiO}$ emission in their surroundings. Some low-mass dense cores could be destroyed as HII regions expand into the clump. Some dense cores in front of HII regions may collide and merge into massive cores or massive filament due to the enhanced density by the HII region shocks. This can explain the lack of widespread low-mass cores in the G9.62+0.19 complex (Liu et al. 2017). New generation of high-mass proto-stars could form in these new massive cores.

Statistical studies of a large number of proto-clusters in the ATOMS survey will help test this scenario.

\subsection{Links to other surveys}

There are several on-going or completed survey programs aiming at studying dense gas in either Galactic Giant molecular clouds or external galaxies. In this section, we will introduce several of these surveys and discuss how they will connect to the ATOMS survey.

The "Millimetre Astronomy Legacy Team $90 \mathrm{GHz}$ (MALT90)" survey (PI: J. M. Jackson) has used the Mopra 22 -m single-dish telescope to obtain $3^{\prime} \times 3^{\prime}$ maps in various gas tracers towards $\sim 2000$ dense molecular clumps that are at different 

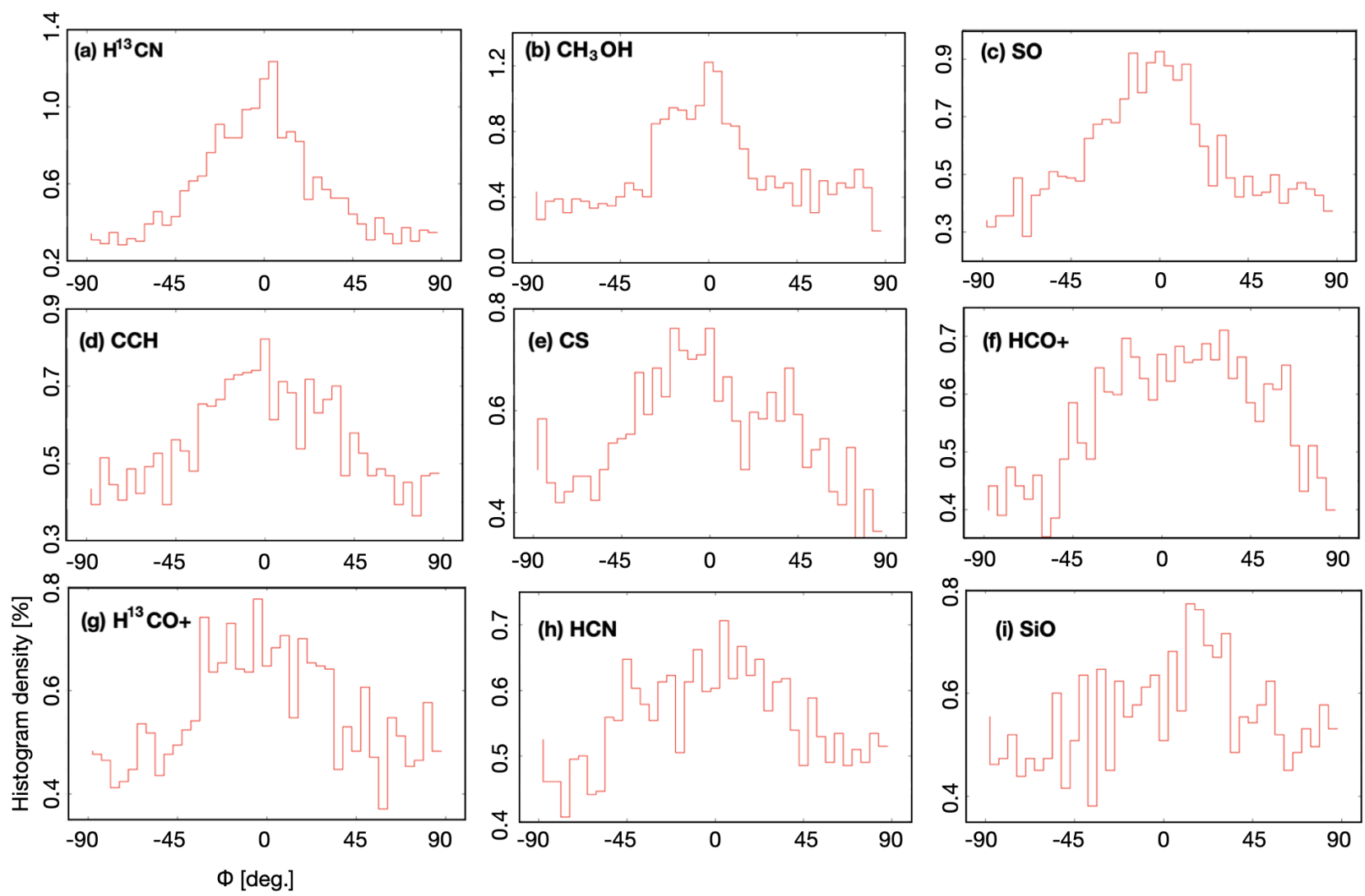

Figure 18. Histogram of relative orientation angles between molecular line emission and $3 \mathrm{~mm}$ continuum emission.

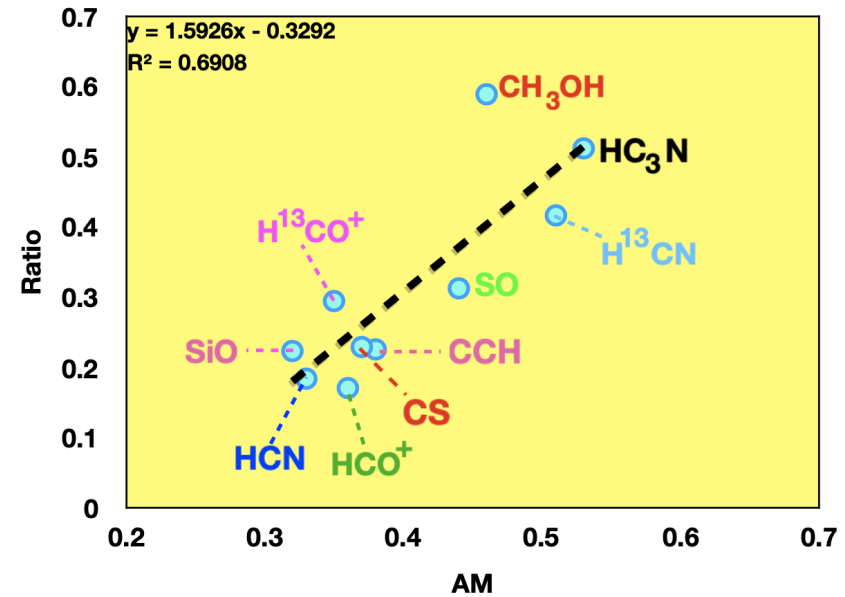

Figure 19. The ratio of $S_{v}^{12 \mathrm{~m}}$ to $S_{v}^{\mathrm{ACA}}$ is plotted as a function of the alignment measurement $(A M)$ in HOG analysis for $3 \mathrm{~mm}$ continuum and molecular line emission.

evolutionary stages (Jackson et al. 2013; Sanhueza et al. 2012; Stephens et al. 2016). The "MALT90" project aims to characterize the physical and chemical evolution of high-mass star-forming clumps. The "ATOMS" survey observed similar gas tracers as in the "MALT90" project, e.g., $\mathrm{HCN}, \mathrm{HCO}^{+}$, and their isotopologue lines, $\mathrm{CCH}, \mathrm{SiO}$, and $\mathrm{HC}_{3} \mathrm{~N}$. The "ATOMS" survey data will be used to study the star formation scaling relations (Liu et al. 2020), and compared with those derived using "MALT90" data (Stephens et al. 2016). The "ATOMS" survey has a much higher angular resolution ( $2^{\prime \prime}$ vs. $\left.38^{\prime \prime}\right)$ than the "MALT90" project. The "ATOMS" survey will reveal the internal structures and detailed spatial gas distributions within the Galactic clumps that cannot be resolved in the "MALT90" project.

The "Orion B: The Anatomy of a Giant Molecular Cloud" (PI: J. Pety) is a multi-line imaging survey at the IRAM 30-m telescope toward the Orion B molecular cloud (Pety et al. 2017). It covers approximately thirty $3 \mathrm{~mm}$ lines with a typical resolution of $26^{\prime \prime}$ (or $\sim 0.05 \mathrm{pc}$ at Orion distance). The "Orion B" project aims to obtain an accurate 3D description of the molecular structure in a GMC and to reveal the detailed anatomy of a molecular emission that is usually hidden behind these galaxy-averaged spectra in nearby galaxy studies. The ATOMS survey has comparable or better spatial resolution $(<0.05 \mathrm{pc})$ than the "Orion B" project for the 98 targeted Galactic clumps within $5 \mathrm{kpc}$. The ATOMS survey aims to reveal the detailed spatial distributions of molecular line emission inside a large sample of Galactic clumps and shares similar science goals as the "Orion B" project. The comparative studies with the "Orion B" data and the "ATOMS" data will shed light on the spatial distributions of molecular emission across a widely different environment from a nearby GMC to distant massive star forming regions.

The "EMIR Multiline Probe of the ISM Regulating Galaxy Evolution" (EMPIRE; PI: Frank Bigiel) survey is a multi-line mapping survey at the IRAM 30-m telescope that is targeting high critical density molecular lines $\left(J=1-0\right.$ of $\mathrm{HCN}, \mathrm{HCO}^{+}$and $\left.\mathrm{HNC}\right)$ and $\mathrm{CO}$ isotopologues across the disks of 9 nearby, star-forming, disk galaxies (Jiménez-Donaire et al. 2019). With an angular resolution of $30^{\prime \prime}$ at $90 \mathrm{GHz}$ (or 1-2kpc), The EMPIRE survey aims to constrain dense gas fraction and star formation efficiency across galaxy 


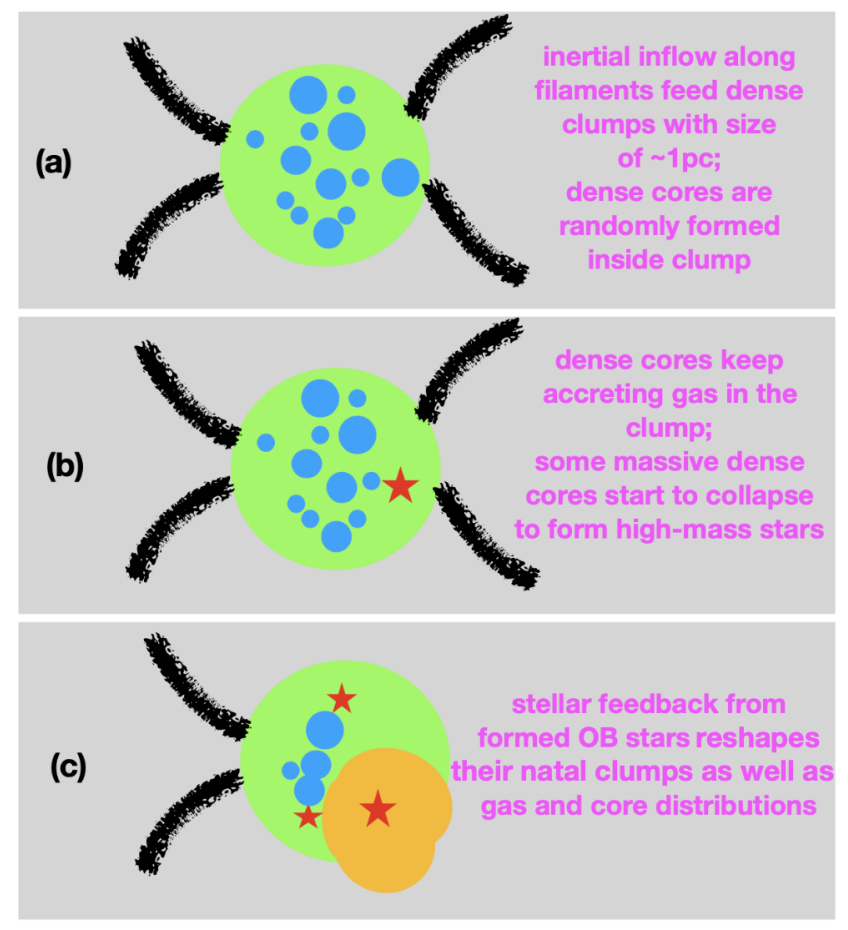

Figure 20. Sketch of the scenario to explain the sequential high-mass star formation in G9.62+0.19. Green filled circle is the massive clump with a typical size of $1 \mathrm{pc}$. The black lines represent converging flows connected to the clump. Blue filled circles are dense cores with a typical size of $0.1 \mathrm{pc}$. Red stars represent formed OB stars. The orange area in panel (c) shows an expanding HII region.

disks and explore how they depend on local conditions (molecular fraction, hydrostatic pressure, radiation field, stellar potential, etc.). The ATOMS survey also aims to investigate the star formation scaling relations with the same high critical density gas tracers, e.g., $J=1-0$ of $\mathrm{HCN}, \mathrm{HCO}^{+}$and their isotopologue lines, and also to study how star formation efficiency changes across the inner Galaxy. The joint analysis of the ATOMS data and "EMPIRE" data will link "dense gas" from the Milky Way to external galaxies.

The "ALMA_IMF: ALMA transforms our view of the origin of stellar masses" (PI: Frédérique Motte) observed 15 most massive clouds within $6 \mathrm{kpc}$ at $3 \mathrm{~mm}$ and $1.3 \mathrm{~mm}$ at the $\sim 2000$ AU spatial resolution. The "ALMA_IMF" project aims to constrain the protocluster core mass function $(\mathrm{CMF})$ in the range $0.5-200 \mathrm{M}_{\odot}$ and to investigate which variables (such as inflows, outflows, or forming filaments) might be correlated with CMF evolution in protoclusters. In contrast, the desired $3 \mathrm{~mm}$ continuum rms level in the ATOMS survey is of $\sim 65 \mu \mathrm{Jy}$, corresponding to a mass sensitivity $\sim 2 \mathrm{M}_{\odot}$ at a distance of $1 \mathrm{kpc}$ and $\sim 50 \mathrm{M}_{\odot}$ at a distance of 5 kpc (Kauffmann et al. 2008). The mass sensitivity is derived with a median dust temperature of $30 \mathrm{~K}$ and a dust opacity per unit gas mass of $0.00189 \mathrm{~cm}^{2} \mathrm{~g}^{-1}$ at $3 \mathrm{~mm}$ that is extrapolated from the dust opacity of $0.01 \mathrm{~cm}^{2} \mathrm{~g}^{-1}$ at $230 \mathrm{GHz}$ assuming a dust opacity in$\operatorname{dex}(\beta)$ of 2 (Ossenkopf\& Henning 1994). Therefore, the ATOMS project will help constrain the high-mass end of the CMFs in protoclusters with a much larger sample but a shallower mass sensitivity than the "ALMA_IMF" project.

Another ALMA survey project, "ALMAGAL: ALMA Evolutionary study of High Mass Protocluster Formation in the Galaxy" (PI: Sergio Molinari), will observe more than 1000 dense clumps with $\mathrm{M}>500 \mathrm{M}_{\odot}$ and $\mathrm{d}<7.5 \mathrm{kpc}$ with similar linear resolution $(\sim 1000 \mathrm{AU})$ and a mass sensitivity of $0.3 \mathrm{M}_{\odot}$ at $1.3 \mathrm{~mm}$. The "ALMAGAL" project will systematically investigate what the physical characteristics of core fragments in dense clumps are as a function of evolution and to what extent fragmentation is driven by dynamics in clumps or mass transport in filaments. The angular resolution and mass sensitivity of the "ALMAGAL" project are much better than the ATOMS project. However, the ATOMS survey benefits from the ( $\sim 3$ times) larger FOV at $3 \mathrm{~mm}$ than that of the "ALMAGAL" project at $1.3 \mathrm{~mm}$, indicating that the ATOMS survey are more suitable to study the overall structures and kinematics in proto-clusters. The comparative studies with the "ALMAGAL" data and the "ATOMS" data will help deepen the understandings of hierarchical fragmentation and differential star formation in highmass proto-clusters.

\section{CONCLUSIONS}

We introduce in this paper the observations, data reduction and example science case, G9.62+0.19 (I18032-2032), of the ALMA survey program ATOMS. The ATOMS project, standing for ALMA Three-millimeter Observations of Massive Star-forming regions, has observed 146 active star forming regions at Band 3 in both continuum emission and molecule lines. More than $90 \%$ of the "ATOMS" targeted sources are promising high-mass star forming regions. The ATOMS survey aims to systematically investigate the spatial distribution of various dense gas tracers, the roles of stellar feedback and filaments in star formation.

(1) The $3 \mathrm{~mm}$ continuum data of the ATOMS survey will be used to detect massive filaments and dense cores. In G9.62+0.19, the $3 \mathrm{~mm}$ continuum emission reveals an expanding HII region "B" to the west and a massive fragmented filament to the east. The 3 mm continuum emission of the expanding HII region "B", compact HII region "MM1/C" and the UC HII region "M11/D" seem to be dominated by free-free emission.

(2) The "ATOMS" survey covers dozens of molecular line transitions, including shocked gas tracers ( $\mathrm{SiO}, \mathrm{SO}$ ), a PDR tracer $(\mathrm{CCH})$, hot core tracers (e.g., $\mathrm{HC}_{3} \mathrm{~N}$ and $\mathrm{CH}_{3} \mathrm{OH}$ ) and some lines commonly assumed to trace dense gas (e.g., $\mathrm{HCN}, \mathrm{HCO}^{+}$and $\mathrm{CS}$ ). It also includes a tracer of ionized gas $\left(\mathrm{H}_{40 \alpha}\right.$ recombination line). We will investigate the spatial distributions of various gas tracers within a large sample of massive clumps. In G9.62+0.19, the similarities and differences in images of 10 molecular lines in Table 2 are investigated with the principal component analysis and the histogram of oriented gradients analysis. We found that the 10 lines can be classified into three groups. The first group includes CS, $\mathrm{HCO}^{+}, \mathrm{HCN}, \mathrm{CCH}$ and $\mathrm{H}^{13} \mathrm{CO}^{+}$, which mainly trace extended gas emission. $\mathrm{SiO}$ in the second group stands out from other molecules because it mainly traces shocked gas. The third group including $\mathrm{SO}, \mathrm{CH}_{3} \mathrm{OH}, \mathrm{H}^{13} \mathrm{CN}$ and $\mathrm{HC}_{3} \mathrm{~N}$ traces well dense gas in the massive filaments. Molecules in the same group show strongest correlations in their spatial distribution. $\mathrm{SO}, \mathrm{CH}_{3} \mathrm{OH}, \mathrm{H}^{13} \mathrm{CN}$ and $\mathrm{HC}_{3} \mathrm{~N}$ show morphology similar to that of the $3 \mathrm{~mm}$ continuum emission. The other molecular lines including the commonly used dense gas tracers, e.g., CS J=2-1, J=1-0 of $\mathrm{HCO}^{+}$and $\mathrm{HCN}$, can not reveal well dense structures (dense cores and filament) in G9.62+0.19 on the scale of $\sim 0.1 \mathrm{pc}$.

(3) In the ATOMS survey, we will for the first time investigate statistically the origin of various velocity shocks using shock tracers like $\mathrm{SiO}$. In G9.62+0.19, SiO shows widespread narrow emis- 
sion, which is likely caused by slow shocks generated by largescale colliding flows and HII regions.

(4) In the ATOMS survey, we will identify outflows driven by proto-stars. For G9.62+0.19, $\mathrm{SiO}, \mathrm{SO}, \mathrm{CS}, \mathrm{HCO}^{+}$and $\mathrm{HCN}$ trace well high-velocity outflow gas emission. The outflows, as traced by these molecular lines, show very similar morphology.

(5) The ATOMS survey covers a wide frequency range that includes dozens of molecular transitions that can be used to study chemical differentiation among dense cores. In G9.62+0.19, three dense cores (MM4/E, MM7/G and MM8/F) show hot core chemistry.

(6) The ATOMS survey aims to statistically evaluate what fraction of gas in gravitationally bound clumps is actually participating in star formation or is concentrated in the smallest star formation units, i.e., dense cores. In G9.62+0.19, only less than $25 \%$ of the "dense gas" revealed by $\mathrm{HCN}, \mathrm{CS}$ and $\mathrm{HCO}^{+}$is concentrated in the massive filament.

(7) The ATOMS survey aims to investigate how stellar feedback from formed OB (proto-)stars in proto-clusters affect their natal clumps and star formation efficiency. Stellar feedback from formed OB stars (HII region "B") in G9.62+0.19 has reshaped their natal clump and changed the spatial distribution of gas and dense cores. Stellar feedback may be also the cause of the sequential highmass star formation that is taking place in G9.62+0.19 complex.

(8) Joint analysis with the ATOMS survey data and other survey data, such as "MALT90", "Orion B", "EMPIRE", "ALMA_IMF", and "ALMAGAL", will help deepen our understandings of "dense gas" star formation scaling relations and massive proto-cluster formation.

\section{ACKNOWLEDGEMENTS}

Tie Liu is supported by the initial funding of scientific research for high-level talents at Shanghai Astronomical Observatory. This work was carried out in part at the Jet Propulsion Laboratory, which is operated for NASA by the California Institute of Technology. LB acknowledges support from CONICYT project Basal AFB170002. Di Li is supported by CAS International Partnership Program No.114A11KYSB20160008. This paper makes use of the following ALMA data: ADS/JAO.ALMA\#2019.1.00685.S. ALMA is a partnership of ESO (representing its member states), NSF (USA) and NINS (Japan), together with NRC (Canada), MOST and ASIAA (Taiwan), and KASI (Republic of Korea), in cooperation with the Republic of Chile. The Joint ALMA Observatory is operated by ESO, AUI/NRAO and NAOJ.

\section{REFERENCES}

Aladro, R., Martín, S., Riquelme, D., et al. 2015, A\&A, 579, A101

Allen, V., van der Tak, F. F. S., Sánchez-Monge, Á., et al., 2017, A\&A, 603A, 133A

André, P., Men'shchikov, A., Bontemps, S., et al., 2010,A\&A, 518L, 102A

André, P.; Di Francesco, J.; Ward-Thompson, D., et al.,2014, Protostars and Planets VI, 914 pp., p.27-51

André, P., Arzoumanian, D., Könyves, V., et al.,2019, A\&A, 629, L4

Arzoumanian, D., André, Ph., Didelon, P., et al., 2011, A\&A, 529L, 6A

Arzoumanian, D., André, P., Könyves, V., et al. 2019, A\&A, 621A, 42A

Berry, D. S. 2015, A\&C, 10, 22

Berry, D. S., Reinhold, K., Jenness, T., \& Economou, F. 2007, in ASP Conf. Ser. 376, Astronomical Data Analysis Software and Systems XVI, ed. R. A. Shaw, F. Hill, \& D. J. Bell (San Francisco, CA: ASP), 425 Barnes, P. J., Hernandez, A. K., O’Dougherty, S. N., 2016, ApJ, 831, 67
Beuther, H., Semenov, D., Henning, T., \& Linz, H. 2008, ApJ, 675, L33

Bigiel, F., Leroy, A., Walter, F., et al. 2008, AJ, 136, 2846

Bonnell, I. A., Bate, M. R., Clarke, C. J., Pringle, J. E., 2002, MNRAS, 323, 785

Bonnell, I. A. \& Bate, M. R., 2006, MNRAS, 370, 488

Bonnell, I. A., 2008, ASPC, 390, 26

Bressert, E., Ginsburg, A., Bally, J., et al. 2012, ApJ, 758, L28

Bronfman, L., Nyman, L. -A., May, J., 1996, A\&AS, 115, 81

Clarke, S. D. \& Whitworth, A. P. 2015, MNRAS, 449, 1819

Chen, H., Gao, Y., Braine, J., et al., 2015, ApJ, 810, 140

Chen, H., Zhang, Q., Wright, M. C. H., et al., 2019, ApJ, 875, 24

Chen, X., Bourke, T. L., Launhardt, R., et al. 2008, ApJ, 686L, 107

Condon, J. J., \& Ransom, S. M. 2016, Essential Radio Astronomy (Princeton, NJ: Princeton Univ. Press)

Contreras, Y., Sanhueza, P., Jackson, J. M., et al., 2018, ApJ, 861, 14

Cosentino, G., Jiménez-Serra, I., Henshaw, J. D., et al., 2018, MNRAS, 474, 3760

Cosentino, G., Jiménez-Serra, I., Caselli, P., et al., 2019, ApJ, 881L, 42C

Cuadrado, S., Goicoechea, J. R., Pilleri, P., et al. 2015, A\&A, 575, A82

Dall'Olio, D., Vlemmings, W. H. T., Persson, M. V., et al., 2019, A\&A, 626A, 36D

Dobbs, C. L., Burkert, A., Pringle, J. E., 2011, MNRAS, 413, 2935

Eden, D. J., Moore, T. J. T., Plume, R., et al. 2017, MNRAS, 469, 2163

Eden, D. J., Liu, T., Kim, K.-T., et al., 2019, MNRAS, 485, 2895

Elmegreen, B. G., \& Lada, C. J. 1977, ApJ, 214, 725

Enoch, M. L., Glenn, J., Evans, N. J., II, et al., 2007, ApJ, 666, 982

Esplugues, G. B., Tercero, B., Cernicharo, J., 2013, A\&A, 556A, 143E

Evans, N. J., II 1999, ARA\&A, 37, 311

Evans, Neal J., II, Heiderman, A., Vutisalchavakul, N., 2014, ApJ, 782, 114

Evans, Neal J., II, Kim, K.-T., Wu, J.-W., 2020, ApJ, 894, 103

Faúndez, S., Bronfman, L., Garay, G., et al. 2004, A\&A, 426, 97

Gao, Y., \& Solomon, P. M. 2004, ApJ, 606, 271

Garay, G., Rodriguez, L. F., Moran, J. M., \& Churchwell, E., 1993, ApJ, 418, 368

Ginsburg, A., Glenn, J., Rosolowsky, E., et al., 2013, ApJS, 208, 14

Goicoechea, J. R., Pety, J., Cuadrado, S., 2016, Nature, 537, 207

González Lobos, V., \& Stutz, A. M. 2019, MNRAS, 489, 4771

Greve, T. R., Leonidaki, I., Xilouris, E. M., et al. 2014, ApJ, 794, 142

Heiderman, A., Evans, N. J. II, Allen, L. E., et al., 2010, ApJ, 723, 1019

Hacar, A., Tafalla, M., Kauffmann, J., et al., 2013, A\&A, 554A, 55H

Hacar, A., Kainulainen, J.; Tafalla, M., et al., 2016, A\&A, 587A, 97H

Hacar, A., Tafalla, M., Forbrich, J., et al., 2018, A\&A, 610A, 77H

Hofner, P., Kurtz, S., Churchwell, E., Walmsley, C. M., \& Cesaroni, R., 1994, ApJ, 429, L85

Hofner, P., Kurtz, S., Churchwell, E., Walmsley, C. M., \& Cesaroni, R., 1996, ApJ, 460, 359

Hofner, P., Wiesemeyer, H., \& Henning, T., 2001, ApJ, 549, 425

Jackson, J. M., Rathborne, J. M., Foster, J. B., 2013, Publ. Astron. Soc. Australia, 30, 57

Jiménez-Serra, I., Caselli, P., Tan, J. C., et al., 2010, MNRAS, 406, 187

Jiménez-Donaire, María J., Bigiel, F., Leroy, A. K., 2019, ApJ, 880, 127

Jones, P. A., Burton, M. G., Cunningham, M. R., et al., 2012, MNRAS, 419, 2961

Jones, P. A., Burton, M. G., Cunningham, M. R., et al., 2013, MNRAS, 433, 221

Juvela, M., Ristorcelli, I., Pagani, L., et al., 2012, 541A, 12J

Kainulainen, J., Stutz, A. M., Stanke, T., et al. 2017, A\&A, 600, A141

Kauffmann, J., Bertoldi, F., Bourke, T. L., 2008, A\&A, 487, 993

Kauffmann, J., \& Pillai, T. 2010, ApJ, 723, L7

Kauffmann, J., Goldsmith, P. F., Melnick, G., et al., 2017, A\&A, 605, L5

Kennicutt, R. C., Jr. 1998a, ApJ, 498, 541

Kennicutt, R. C., Jr. 1998b, ARA\&A, 36, 189

Kennicutt, R. C., \& Evans, N. J. 2012, ARA\&A, 50, 531

Kirk, H., Myers, P. C., Bourke, T. L., et al. 2013, ApJ, 766, 115

Krumholz, M. R., \& McKee, C. F. 2008, Nature, 451, 1082

Krumholz, M. R., Klein, R. I., McKee, C. F., 2011, ApJ, 740, 74

Krumholz, M. R., Bate, M. R., Arce, H. G., et al., 2014, Protostars and Planets VI, Henrik Beuther, Ralf S. Klessen, Cornelis P. Dullemond, 
and Thomas Henning (eds.), University of Arizona Press, Tucson, 914 pp., p. 243-266

Hoq, S., Jackson, J. M., Foster, J. B., et al., 2013, ApJ, 777, 157

Könyves, V., André, Ph., Men'shchikov, A., et al., 2015, A\&A, 584A, 91K

Lada, C. J., Forbrich, J., Lombardi, M., et al. 2012, ApJ, 745, 190

Lada, C. J., Lombardi, M., \& Alves, J. F. 2010, ApJ, 724, 687

Lazarian, A., \& Hoang, T. 2007, MNRAS, 378, 910

Le Page, V., Snow, T. P., \& Bierbaum, V. M. 2003, ApJ, 584, 316

Liu, D., Gao, Y., Isaak, K., et al. 2015, ApJ, 810L, 14L

Liu, H. B., Jiménez-Serra, I., Ho, P. T. P., et al. 2012, ApJ, 756, 10

Liu, H. B., Galván-Madrid, R., Jiménez-Serra, I., et al. 2015b, ApJ, 804, 37

Liu, H. B., Chen, H.-R. V., Román-Zúñiga, C. G., 2019, ApJ, 871, 185

Liu, T., Wu, Y., Liu, S.-Y., et al. 2011, ApJ, 730, 102

Liu, T., Kim, K.-T., Yoo, H., et al., 2016, ApJ, 829, 59

Liu, T., Lacy, J., Li, P. S., et al., 2017, ApJ, 849, 25

Liu, T., Kim, K.-T., Juvela, M., et al., 2018a, ApJS, 234, 28L

Liu, T., Li, P. S., Juvela, M., et al., 2018b, ApJ, 859, 151L

Liu T., et al., 2018, ApJ, 869, L5

Liu, T., Evans, N., Kim, K.-T., et al., 2020, accepted to MNRAS, 496, 2790

Lo, N., Cunningham, M. R., Jones, P. A., et al., 2009, 395, 1021

Louvet, F., Motte, F., Gusdorf, A., et al., 2016, A\&A, 595A, 122L

Lu, X., Zhang, Q., Liu, H. B., et al. 2018, ApJ, 855, 9

Moscadelli, L., Rivilla, V. M., Cesaroni, R., et al., 2018, A\&A, 616A, 66M

Molinari, S., Pezzuto, S., Cesaroni, R., et al. 2008, A\&A, 481, 345

Molinari, S., Swinyard, B., Bally, J., et al., 2010, A\&A, 518L, 100M

McMullin, J. P., Waters, B., Schiebel, D., et al. 2007, adass XVI, 127

Möller, T., Endres, C., Schilke, P., 2017, A\&A, 598, A7

Montillaud, J., Joblin, C., \& Toublanc, D. 2013, A\&A, 552, A15

Myers, A. T., McKee, C. F., Cunningham, A. J., et al., 2013, ApJ, 766, 97

Nagy, Z., Spezzano, S., Caselli, P., et al., 2019, A\&A, 630A, 136N

Nguyen-Lu'o'ng, Q., Motte, F., Carlhoff, P., et al., 2013, ApJ, 775, 88

Nguyen-Luong, Q., Nakamura, F., Sugitani, K., et al., 2020, ApJ, 891, 66

Offner, S. S. R., Klein, R. I., McKee, C. F., 2009, ApJ, 703, 131

Ossenkopf, V., \& Henning, T. 1994, A\&A, 291, 943

Padoan, P., Pan, L., Juvela, M., et al., 2019, ApJ, in press, arXiv:1911.04465

Papadopoulos, Padeli P. 2007, ApJ, 656, 792

Peng, Y., Rivilla, V. M., Zhang, L., et al., 2019, ApJ, 871, 251

Peng, Y., Qin, S.-L., Schilke, P., et al., 2017, ApJ, 837, 49

Peretto, N., Fuller, G. A., Duarte-Cabral, A., et al. 2013, A\&A, 555, 112

Pety, J., Teyssier, D., Fossé, D., et al. 2005, A\&A, 435, 885

Pety, J., Guzmán, V. V., Orkisz, J. H., et al., 2017, A\&A, 599, A98

Pols, S., Schwörer, A., Schilke, P., et al., 2018, A\&A, 614A, 123P

Pon, A., Johnstone, D., \& Heitsch, F. 2011, ApJ, 740, 88

Pon, A., ToalÃą, J. A., Johnstone, D., et al. 2012, ApJ, 756, 145

Pratap, P., Dickens, J. E., Snell, R. L., et al. 1997, ApJ, 486, 862

Qin, S.-L., Schilke, P., Wu, J., et al., 2015, ApJ, 803, 39

Quireza, C., Rood, R. T.; Bania, T. M., et al., 2006, ApJ, 653, 1226

Sanna, A., Reid, M. J., Moscadelli, L., Dame, T. M., Menten, K. M., Brunthaler, A., Zheng, X. W., Xu, Y., 2009, ApJ, 706, 464

Sanhueza, P., Jackson, J. M., Foster, J. B., et al., 2012, ApJ, 756, 60

Sanhueza, P., Contreras, Y., Wu, B., et al., 2019, ApJ, 886, 102

Saraceno, P., Andre, P., Ceccarelli, C., Griffin, M., \& Molinari, S. 1996, A\&A, 309, 827

Schisano, E., Molinari, S., Elia, D., et al., 2020, MNRAS, 492, 5420

Schmidt, M. 1959, ApJ, 129, 243

Silk, J. 1997, ApJ, 481, 703

Shimajiri, Y., André, P., Braine, J., et al., 2017, A\&A, 604, A74

Shirley, Yancy L., 2015, PASP, 127, 299

Soler, J. D., Beuther, H., Rugel, M.,et al., 2019, A\&A, 622A, 166S

Solomon, P. M., Downes, D., Radford, S. J. E., \& Barrett, J. W. 1997, ApJ, 478,144

Spezzano, S., Caselli, P., Bizzocchi, L., 2017, A\&A, 606A, 82S

Stephens, I. W., Jackson, J. M., \& Whitaker, J. S. 2016, ApJ, 824, 29

Stutz, A. M., \& Gould, A. 2016, A\&A, 590, A2

Stutz, A. M. 2018, MNRAS, 473, 4890

Tacconi, L. J., Genzel, R., \& Sternberg, A., 2020, ARA\&A, in press, eprint arXiv:2003.06245

Testi, L., Hofner, P., Kurtz, S., \& Rupen, M., 2000, A\&A, 359, L5
Tiwari, M., Menten, K. M., Wyrowski, F., et al., 2019, A\&A, 626A, 28T

Thompson, M. A., Urquhart, J. S., Moore, T. J. T., et al., 2012, MNRAS, 421, 408

Tan, Q., Gao, Y., Zhang, Z.-Y. et al., 2018, ApJ, 860, 165

Tobin, J. J., Bourke, T. L., Mader, S., 2019, ApJ, 870, 81

Urquhart, J. S., Moore, T. J. T., Csengeri, T., et al. 2014, MNRAS, 443, 1555

Urquhart, J. S., König, C., Giannetti, A., 2018, MNRAS, 473, 1059

Vutisalchavakul, N., Evans, N. J., II, Heyer, M., 2016, ApJ, 831, 73

Wang, K., Zhang, Q., Wu, Y., 2011, ApJ, 735, 64W

Wang, K., Zhang, Q., Testi, L., et al., 2014, MNRAS, 439, 3275

Wang, K., Zhang, Q., Wu, Y., 2012, ApJ, 745L, 30

Wang, K., Testi, L., Burkert, A., et al. 2016, ApJS, 226, 9

Wall, J. E., Mac Low, M., McMillan, S. L. W., et al., 2020, submitted, arXiv:2003.09011

Whitworth, A. P., Bhattal, A. S., Chapman, S. J., et al., 1994a, A\&A, 290, 421

Whitworth, A. P., Bhattal, A. S., Chapman, S. J., et al., 1994b, MNRAS, 268, 291

Wu, J., Evans, N. J., II, Gao, Y., et al. 2005, ApJ, 635, L173

Wu, J., Evans, N. J., II, Shirley, Y. L., et al. 2010, ApJS, 188, 313

Yuan, J., Wu, Y., Ellingsen, S. P., et al., 2017, ApJS, 231, 11

Zhang, Q., \& Wang, K. 2011, ApJ, 733, 26

Zhang, Q., Wang, K., Lu, X., 2015, ApJ, 804, 141

Zhang, Z.-Y., Gao, Y., Henkel, C., et al. 2014, ApJL, 784, L31

Zhu, F.-Y., Zhu, Q.-F., Wang, J.-Z., et al., 2019, ApJ, 881, 14

\section{APPENDIX A:}


Table A1. Basic parameters of the targets in the ATOMS survey.

\begin{tabular}{|c|c|c|c|c|c|c|c|c|c|c|c|}
\hline ID & IRAS & RA & DEC & $\begin{array}{c}\mathrm{V}_{\mathrm{lsr}} \\
\left(\mathrm{km} \mathrm{s}^{-1}\right)\end{array}$ & $\begin{array}{c}\text { Distance } \\
(\mathrm{kpc})\end{array}$ & $\begin{array}{l}R_{\mathrm{GC}} \\
(\mathrm{kpc})\end{array}$ & $\begin{array}{c}\text { Radius } \\
\text { (pc) }\end{array}$ & $\begin{array}{c}T_{\text {dust }} \\
(\mathrm{K})\end{array}$ & $\begin{array}{c}\log \left(L_{\mathrm{bol}}\right) \\
\left(\mathrm{L}_{\odot}\right)\end{array}$ & $\begin{array}{c}\log \left(M_{\text {clump }}\right) \\
\left(\mathrm{M}_{\odot}\right)\end{array}$ & $\operatorname{refs}^{a}$ \\
\hline 1 & I08076-3556 & 08:09:32.39 & $-36: 05: 13.2$ & 5.9 & 0.4 & 8.5 & 0.07 & 18.0 & 1.2 & 0.7 & 1 \\
\hline 2 & I08303-4303 & 08:32:08.34 & $-43: 13: 54.0$ & 14.3 & 2.3 & 9.0 & 0.32 & 30.0 & 3.8 & 2.4 & 1 \\
\hline 3 & I08448-4343 & $08: 46: 32.90$ & $-43: 54: 35.9$ & 3.7 & 0.7 & 8.4 & 0.15 & 25.0 & 3.0 & 1.6 & 1 \\
\hline 4 & I08470-4243 & 08:48:47.07 & $-42: 54: 31.0$ & 12 & 2.1 & 8.8 & 0.32 & 33.0 & 4.0 & 2.4 & 1 \\
\hline 5 & I09002-4732 & 09:01:54.24 & $-47: 44: 00.8$ & 3.1 & 1.2 & 8.4 & 0.24 & 39.0 & 4.6 & 2.4 & 1 \\
\hline 6 & I09018-4816 & 09:03:32.84 & $-48: 28: 10.0$ & 10.3 & 2.6 & 8.8 & 0.44 & 31.0 & 4.7 & 3.0 & 1 \\
\hline 7 & I09094-4803 & 09:11:07.29 & $-48: 15: 48.7$ & 74.6 & 9.6 & 12.7 & 1.40 & 23.0 & 4.6 & 3.1 & 1 \\
\hline 8 & I10365-5803 & $10: 38: 32.46$ & $-58: 19: 05.9$ & -19 & 2.4 & 8.0 & 0.44 & 30.0 & 4.3 & 2.7 & 1 \\
\hline 9 & I11298-6155 & $11: 32: 05.70$ & $-62: 12: 24.3$ & 32.9 & 10 & 10.1 & 1.36 & 32.0 & 5.2 & 3.4 & 1 \\
\hline 10 & I1 1332-6258 & $11: 35: 31.81$ & $-63: 14: 44.6$ & -15.4 & 1.9 & 7.7 & 0.28 & 30.0 & 3.7 & 2.1 & 1 \\
\hline 11 & I1 1590-6452 & $12: 01: 34.54$ & $-65: 08: 52.9$ & -4.3 & 0.4 & 8.2 & 0.06 & 22.0 & 1.7 & 1.1 & 1 \\
\hline 12 & I12320-6122 & $12: 34: 53.38$ & $-61: 39: 46.9$ & -42.5 & 3.43 & 7.2 & 1.00 & 44.6 & 5.6 & 3.0 & 2 \\
\hline 13 & I12326-6245 & $12: 35: 34.81$ & $-63: 02: 32.1$ & -39.6 & 4.61 & 7.2 & 0.83 & 34.2 & 5.4 & 3.5 & 2 \\
\hline 14 & I12383-6128 & $12: 41: 17.32$ & $-61: 44: 38.6$ & -39.1 & 3.27 & 7.2 & 0.63 & 19.8 & 3.8 & 3.1 & 2 \\
\hline 15 & I12572-6316 & $13: 00: 24.43$ & $-63: 32: 30.4$ & 30.9 & 11.57 & 9.8 & 1.63 & 21.5 & 4.6 & 3.9 & 2 \\
\hline 16 & I13079-6218 & $13: 11: 14.16$ & $-62: 34: 42.1$ & -42.6 & 3.8 & 6.9 & 1.09 & 28.2 & 5.1 & 3.5 & 2 \\
\hline 17 & I13080-6229 & 13:11:14.10 & $-62: 45: 01.9$ & -35.6 & 3.8 & 6.9 & 1.03 & 34.1 & 5.1 & 3.2 & 2 \\
\hline 18 & I13111-6228 & $13: 14: 26.43$ & $-62: 44: 24.1$ & -38.8 & 3.8 & 6.9 & 0.96 & 30.0 & 4.8 & 3.1 & 2 \\
\hline 19 & I13134-6242 & $13: 16: 43.05$ & $-62: 58: 30.1$ & -31.5 & 3.8 & 6.9 & 0.57 & 29.2 & 4.6 & 3.1 & 2 \\
\hline 20 & I13140-6226 & $13: 17: 15.70$ & $-62: 42: 27.5$ & -33.9 & 3.8 & 6.9 & 0.50 & 22.6 & 3.8 & 2.9 & 2 \\
\hline 21 & I13291-6229 & $13: 32: 32.38$ & $-62: 45: 23.5$ & -36.5 & 2.9 & 7.0 & 0.55 & 28.8 & 4.3 & 2.7 & 2 \\
\hline 22 & I13291-6249 & $13: 32: 31.23$ & $-63: 05: 21.8$ & -34.7 & 7.61 & 7.1 & 1.73 & 27.2 & 5.2 & 3.7 & 2 \\
\hline 23 & I13295-6152 & $13: 32: 53.49$ & $-62: 07: 49.3$ & -44.4 & 3.89 & 6.7 & 0.36 & 19.4 & 3.3 & 2.7 & 2 \\
\hline 24 & I13471-6120 & $13: 50: 42.10$ & $-61: 35: 14.9$ & -56.7 & 5.46 & 6.4 & 1.01 & 35.1 & 5.3 & 3.4 & 2 \\
\hline 25 & I13484-6100 & $13: 51: 58.64$ & $-61: 15: 43.3$ & -55 & 5.4 & 6.4 & 0.84 & 31.8 & 4.8 & 3.1 & 2 \\
\hline 26 & I14013-6105 & $14: 04: 54.56$ & $-61: 20: 10.7$ & -48.1 & 4.12 & 6.4 & 0.80 & 31.4 & 5.0 & 3.2 & 2 \\
\hline 27 & I14050-6056 & $14: 08: 42.15$ & $-61: 10: 43.0$ & -47.1 & 3.42 & 6.6 & 0.66 & 32.2 & 4.7 & 2.8 & 2 \\
\hline 28 & I14164-6028 & $14: 20: 08.23$ & $-60: 42: 05.0$ & -46.5 & 3.19 & 6.6 & 0.17 & 28.7 & 3.7 & 2.2 & 2 \\
\hline 29 & I14206-6151 & $14: 24: 22.81$ & $-62: 05: 22.7$ & -50 & 3.29 & 6.5 & 0.37 & 27.3 & 3.7 & 2.4 & 2 \\
\hline 30 & I14212-6131 & $14: 25: 01.08$ & $-61: 44: 59.4$ & -50.5 & 3.44 & 6.5 & 0.57 & 21.8 & 4.0 & 3.0 & 2 \\
\hline 31 & I14382-6017 & $14: 42: 02.76$ & $-60: 30: 35.1$ & -60.7 & 7.69 & 6.0 & 1.68 & 28.0 & 5.2 & 3.6 & 2 \\
\hline 32 & I14453-5912 & 14:49:07.77 & $-59: 24: 49.7$ & -40.2 & 2.82 & 6.6 & 0.79 & 24.1 & 4.2 & 2.9 & 2 \\
\hline 33 & I14498-5856 & $14: 53: 42.81$ & $-59: 08: 56.5$ & -49.3 & 3.16 & 6.4 & 0.74 & 26.7 & 4.4 & 3.0 & 2 \\
\hline 34 & I15122-5801 & $15: 16: 06.77$ & $-58: 11: 40.5$ & -60.9 & 9.26 & 5.9 & 2.65 & 27.7 & 5.0 & 3.9 & 2 \\
\hline 35 & I15254-5621 & $15: 29: 19.48$ & $-56: 31: 23.2$ & -67.3 & 4 & 5.7 & 0.89 & 33.5 & 5.1 & 3.1 & 2 \\
\hline 36 & I15290-5546 & $15: 32: 53.16$ & $-55: 56: 06.8$ & -87.5 & 6.76 & 4.9 & 1.80 & 33.5 & 5.7 & 3.8 & 2 \\
\hline 37 & I15384-5348 & $15: 42: 16.57$ & $-53: 58: 31.3$ & -41 & 1.82 & 6.9 & 0.54 & 33.9 & 4.6 & 2.5 & 2 \\
\hline 38 & I15394-5358 & $15: 43: 16.48$ & $-54: 07: 16.9$ & -41.6 & 1.82 & 6.9 & 0.46 & 20.7 & 3.7 & 2.9 & 2 \\
\hline 39 & I15408-5356 & $15: 44: 43.00$ & $-54: 05: 44.9$ & -39.7 & 1.82 & 6.9 & 0.61 & 34.0 & 4.9 & 2.9 & 2 \\
\hline 40 & I15411-5352 & $15: 44: 59.59$ & $-54: 02: 21.4$ & -41.5 & 1.82 & 6.9 & 0.57 & 30.5 & 4.5 & 2.7 & 2 \\
\hline 41 & I15437-5343 & $15: 47: 33.11$ & $-53: 52: 43.9$ & -83 & 4.98 & 5.0 & 0.77 & 29.6 & 4.6 & 3.0 & 2 \\
\hline 42 & I15439-5449 & $15: 47: 49.82$ & $-54: 58: 32.1$ & -54.6 & 3.29 & 5.9 & 0.69 & 26.8 & 4.4 & 3.0 & 2 \\
\hline 43 & I15502-5302 & $15: 54: 06.43$ & $-53: 11: 38.4$ & -91.4 & 5.8 & 4.6 & 1.66 & 35.7 & 5.8 & 3.7 & 2 \\
\hline 44 & I15520-5234 & $15: 55: 48.84$ & $-52: 43: 06.2$ & -41.3 & 2.65 & 6.2 & 0.67 & 32.2 & 5.1 & 3.2 & 2 \\
\hline 45 & I15522-5411 & $15: 56: 07.74$ & $-54: 19: 57.8$ & -46.7 & 2.73 & 6.2 & 0.64 & 23.3 & 3.8 & 2.9 & 2 \\
\hline 46 & I15557-5215 & 15:59:40.76 & $-52: 23: 27.7$ & -67.6 & 4.03 & 5.3 & 0.82 & 20.7 & 3.9 & 3.2 & 2 \\
\hline 47 & I15567-5236 & $16: 00: 32.86$ & $-52: 44: 45.1$ & -107.1 & 5.99 & 4.4 & 1.31 & 35.4 & 5.7 & 3.5 & 2 \\
\hline 48 & I15570-5227 & $16: 00: 55.56$ & $-52: 36: 21.2$ & -101.5 & 5.99 & 4.4 & 1.66 & 28.7 & 4.8 & 3.4 & 2 \\
\hline 49 & I15584-5247 & $16: 02: 19.63$ & $-52: 55: 22.4$ & -76.8 & 4.41 & 5.1 & 0.86 & 23.9 & 4.2 & 3.1 & 2 \\
\hline 50 & I15596-5301 & $16: 03: 32.29$ & $-53: 09: 28.1$ & -72.1 & 10.11 & 5.2 & 1.81 & 28.5 & 5.5 & 3.9 & 2 \\
\hline 51 & I16026-5035 & $16: 06: 25.43$ & $-50: 43: 07.2$ & -78.3 & 4.53 & 4.9 & 0.81 & 31.8 & 4.9 & 3.0 & 2 \\
\hline 52 & I16037-5223 & $16: 07: 38.10$ & $-52: 31: 00.2$ & -80 & 9.84 & 4.9 & 2.15 & 31.4 & 5.6 & 3.8 & 2 \\
\hline 53 & I16060-5146 & 16:09:52.85 & $-51: 54: 54.7$ & -91.6 & 5.3 & 4.5 & 1.24 & 32.2 & 5.8 & 3.9 & 2 \\
\hline 54 & I16065-5158 & $16: 10: 20.30$ & $-52: 06: 07.1$ & -63.3 & 3.98 & 5.2 & 1.41 & 30.8 & 5.4 & 3.7 & 2 \\
\hline 55 & I16071-5142 & 16:11:00.01 & $-51: 50: 21.6$ & -87 & 5.3 & 4.5 & 1.21 & 23.9 & 4.8 & 3.7 & 2 \\
\hline 56 & I16076-5134 & $16: 11: 27.12$ & $-51: 41: 56.9$ & -87.7 & 5.3 & 4.5 & 1.57 & 30.1 & 5.3 & 3.6 & 2 \\
\hline 57 & I16119-5048 & $16: 15: 45.65$ & $-50: 55: 53.5$ & -48.2 & 3.1 & 5.8 & 0.93 & 24.0 & 4.3 & 3.2 & 2 \\
\hline 58 & I16132-5039 & $16: 17: 01.52$ & $-50: 46: 51.0$ & -47.5 & 3.1 & 5.8 & 1.04 & 32.5 & 4.6 & 2.9 & 2 \\
\hline 59 & I16158-5055 & $16: 19: 38.63$ & $-51: 03: 20.0$ & -49.2 & 3.57 & 5.4 & 1.35 & 28.3 & 5.2 & 3.5 & 2 \\
\hline 60 & I16164-5046 & $16: 20: 10.91$ & $-50: 53: 15.5$ & -57.3 & 3.57 & 5.4 & 1.37 & 31.4 & 5.5 & 3.7 & 2 \\
\hline 61 & I16172-5028 & $16: 21: 02.47$ & $-50: 35: 10.3$ & -51.9 & 3.57 & 5.4 & 1.51 & 32.0 & 5.8 & 4.0 & 2 \\
\hline 62 & I16177-5018 & $16: 21: 31.49$ & $-50: 25: 04.5$ & -50.2 & 3.57 & 5.4 & 1.25 & 34.0 & 5.5 & 3.6 & 2 \\
\hline
\end{tabular}

${ }^{a}$ Ref 1: Faúndez et al. (2004); Ref 2: Urquhart et al. (2018) 
Table A1 - continued Basic parameters of the targets in the ATOMS survey.

\begin{tabular}{|c|c|c|c|c|c|c|c|c|c|c|c|}
\hline Number & IRAS & RA & DEC & $\begin{array}{c}V_{\mathrm{lsr}} \\
\left(\mathrm{km} \mathrm{s}^{-1}\right)\end{array}$ & $\begin{array}{c}\text { Distance } \\
(\mathrm{kpc})\end{array}$ & $\begin{array}{l}R_{\mathrm{GC}} \\
(\mathrm{kpc})\end{array}$ & $\begin{array}{c}\text { Radius } \\
\text { (pc) }\end{array}$ & $\begin{array}{l}T_{\text {dust }} \\
(\mathrm{K})\end{array}$ & $\begin{array}{c}\log \left(L_{\text {bol }}\right) \\
\left(\mathrm{L}_{\odot}\right)\end{array}$ & $\begin{array}{c}\log \left(M_{\text {clump }}\right) \\
\quad\left(\mathrm{M}_{\odot}\right)\end{array}$ & refs \\
\hline 63 & I16272-4837 & $16: 30: 59.08$ & $-48: 43: 53.3$ & -46.6 & 2.92 & 5.8 & 0.84 & 23.1 & 4.3 & 3.2 & 2 \\
\hline 64 & I16297-4757 & $16: 33: 29.45$ & $-48: 03: 41.2$ & -79.6 & 5.03 & 4.2 & 2.00 & 27.1 & 4.9 & 3.4 & 2 \\
\hline 65 & I16304-4710 & $16: 34: 05.12$ & $-47: 16: 32.6$ & -62.8 & 11.32 & 4.9 & 1.37 & 27.5 & 4.9 & 3.5 & 2 \\
\hline 66 & I16313-4729 & $16: 34: 54.98$ & $-47: 35: 35.0$ & -73.7 & 4.71 & 4.4 & 2.06 & 31.0 & 6.7 & 4.7 & 1,2 \\
\hline 67 & I16318-4724 & $16: 35: 34.11$ & $-47: 31: 11.3$ & -119.8 & 7.68 & 3.3 & 1.45 & 29.5 & 5.2 & 3.7 & 2 \\
\hline 68 & I16330-4725 & $16: 36: 42.96$ & $-47: 31: 29.7$ & -75.1 & 10.99 & 4.6 & 3.89 & 33.8 & 6.3 & 4.3 & 2 \\
\hline 69 & I16344-4658 & $16: 38: 09.38$ & $-47: 04: 58.7$ & -49.5 & 12.09 & 5.4 & 2.70 & 25.5 & 5.4 & 4.1 & 2 \\
\hline 70 & I16348-4654 & $16: 38: 29.64$ & $-47: 00: 41.1$ & -46.5 & 12.09 & 5.4 & 2.40 & 23.6 & 5.4 & 4.4 & 2 \\
\hline 71 & I16351-4722 & $16: 38: 50.98$ & $-47: 27: 57.8$ & -41.4 & 3.02 & 5.7 & 0.69 & 30.4 & 4.9 & 3.2 & 2 \\
\hline 72 & I16362-4639 & $16: 39: 57.32$ & $-46: 45: 06.3$ & -38.8 & 3.01 & 5.7 & 0.54 & 24.0 & 3.6 & 2.5 & 2 \\
\hline 73 & I16372-4545 & $16: 40: 54.57$ & $-45: 50: 49.6$ & -57.3 & 4.16 & 4.7 & 0.73 & 26.0 & 4.2 & 2.9 & 2 \\
\hline 74 & I16385-4619 & $16: 42: 14.04$ & $-46: 25: 25.9$ & -117 & 7.11 & 3.1 & 1.34 & 31.9 & 5.1 & 3.2 & 2 \\
\hline 75 & I16424-4531 & $16: 46: 06.61$ & $-45: 36: 46.6$ & -34.2 & 2.63 & 6.0 & 0.54 & 24.6 & 3.9 & 2.7 & 2 \\
\hline 76 & I16445-4459 & $16: 48: 05.18$ & $-45: 05: 08.6$ & -121.3 & 7.95 & 2.8 & 2.54 & 24.6 & 5.0 & 3.9 & 2 \\
\hline 77 & I16458-4512 & $16: 49: 30.41$ & $-45: 17: 53.6$ & -50.4 & 3.56 & 5.1 & 1.42 & 21.4 & 4.5 & 3.6 & 2 \\
\hline 78 & I16484-4603 & $16: 52: 04.29$ & $-46: 08: 30.1$ & -32 & 2.1 & 6.4 & 0.55 & 35.0 & 5.0 & 3.0 & 1,2 \\
\hline 79 & I16487-4423 & $16: 52: 23.67$ & $-44: 27: 52.3$ & -43.4 & 3.26 & 5.4 & 1.01 & 24.6 & 4.4 & 3.0 & 2 \\
\hline 80 & I16489-4431 & $16: 52: 33.50$ & $-44: 36: 17.7$ & -41.3 & 3.26 & 5.4 & 0.71 & 21.8 & 3.8 & 2.9 & 2 \\
\hline 81 & I16506-4512 & $16: 54: 15.77$ & $-45: 17: 31.8$ & -26.2 & 2.42 & 6.1 & 0.86 & 32.4 & 5.0 & 3.1 & 2 \\
\hline 82 & I16524-4300 & $16: 56: 03.06$ & $-43: 04: 43.3$ & -40.8 & 3.43 & 5.2 & 1.06 & 23.4 & 4.4 & 3.4 & 2 \\
\hline 83 & I16547-4247 & $16: 58: 17.26$ & $-42: 52: 04.5$ & -30.4 & 2.74 & 5.8 & 0.69 & 28.9 & 4.8 & 3.2 & 2 \\
\hline 84 & I16562-3959 & $16: 59: 41.42$ & $-40: 03: 46.6$ & -12.6 & 2.38 & 6.1 & 0.72 & 42.3 & 5.7 & 3.2 & 2 \\
\hline 85 & I16571-4029 & $17: 00: 32.21$ & $-40: 34: 12.7$ & -15 & 2.38 & 6.1 & 0.38 & 27.0 & 4.3 & 2.9 & 2 \\
\hline 86 & I17006-4215 & 17:04:12.99 & $-42: 19: 54.2$ & -23.2 & 2.21 & 6.3 & 0.50 & 27.7 & 4.4 & 2.8 & 2 \\
\hline 87 & I17008-4040 & $17: 04: 23.03$ & $-40: 44: 24.9$ & -17 & 2.38 & 6.1 & 1.26 & 31.0 & 4.6 & 3.0 & 1,2 \\
\hline 88 & I17016-4124 & $17: 05: 11.02$ & $-41: 29: 07.8$ & -27.1 & 1.37 & 7.0 & 0.75 & 32.0 & 5.3 & 3.8 & 1,2 \\
\hline 89 & I17136-3617 & $17: 17: 02.04$ & $-36: 20: 52.5$ & -10.6 & 1.34 & 7.0 & 0.45 & 34.8 & 4.6 & 2.5 & 2 \\
\hline 90 & I17143-3700 & $17: 17: 45.65$ & $-37: 03: 11.8$ & -31.1 & 12.67 & 4.7 & 2.95 & 31.0 & 5.6 & 3.8 & 2 \\
\hline 91 & I17158-3901 & $17: 19: 20.34$ & $-39: 03: 53.3$ & -15.2 & 3.38 & 5.1 & 1.18 & 23.3 & 4.8 & 3.4 & 2 \\
\hline 92 & I17160-3707 & $17: 19: 26.81$ & $-37: 11: 01.4$ & -69.5 & 10.53 & 2.7 & 1.69 & 28.5 & 6.0 & 4.1 & 2 \\
\hline 93 & I17175-3544 & $17: 20: 53.10$ & $-35: 47: 03.0$ & -5.7 & 1.34 & 7.0 & 0.35 & 30.6 & 4.8 & 3.1 & 2 \\
\hline 94 & I17204-3636 & $17: 23: 50.32$ & $-36: 38: 58.1$ & -18.2 & 3.32 & 5.1 & 0.60 & 25.8 & 4.2 & 2.9 & 2 \\
\hline 95 & I17220-3609 & $17: 25: 24.99$ & $-36: 12: 45.1$ & -93.7 & 8.01 & 1.3 & 2.41 & 25.4 & 5.7 & 4.3 & 2 \\
\hline 96 & I17233-3606 & $17: 26: 42.73$ & $-36: 09: 20.8$ & -2.7 & 1.34 & 7.0 & 0.37 & 29.9 & 4.6 & 3.0 & 2 \\
\hline 97 & I17244-3536 & $17: 27: 48.71$ & $-35: 39: 10.8$ & -10.2 & 1.36 & 7.0 & 0.30 & 29.8 & 3.7 & 2.1 & 2 \\
\hline 98 & I17258-3637 & $17: 29: 16.99$ & $-36: 40: 16.5$ & -11.9 & 2.59 & 5.8 & 0.92 & 45.5 & 5.9 & 3.3 & 2 \\
\hline 99 & I17269-3312 & $17: 30: 14.77$ & $-33: 14: 57.5$ & -21 & 4.38 & 4.0 & 1.53 & 22.4 & 4.7 & 3.6 & 2 \\
\hline 100 & I17271-3439 & $17: 30: 26.21$ & $-34: 41: 48.9$ & -18.2 & 3.1 & 5.3 & 1.34 & 35.0 & 5.6 & 4.0 & 1,2 \\
\hline 101 & I17278-3541 & $17: 31: 14.07$ & $-35: 44: 07.3$ & -0.4 & 1.33 & 7.0 & 0.31 & 25.1 & 3.8 & 2.5 & 2 \\
\hline 102 & I17439-2845 & $17: 47: 09.20$ & $-28: 46: 13.9$ & 18.7 & 8 & 0.3 & 1.78 & 30.0 & 5.7 & 3.8 & 1 \\
\hline 103 & I17441-2822 & $17: 47: 19.79$ & $-28: 23: 05.7$ & 50.8 & 8.1 & 0.2 & 2.51 & 35.0 & 6.6 & 5.4 & 1 \\
\hline 104 & I1 $17455-2800$ & $17: 48: 41.63$ & $-28: 01: 44.6$ & -15.6 & 10 & 1.7 & 2.18 & 31.0 & 5.9 & 4.1 & 1 \\
\hline 105 & I17545-2357 & $17: 57: 34.49$ & $-23: 58: 04.3$ & 7.9 & 2.93 & 5.4 & 0.87 & 23.7 & 4.1 & 3.1 & 2 \\
\hline 106 & I17589-2312 & $18: 01: 57.87$ & $-23: 12: 32.9$ & 21.3 & 2.97 & 5.4 & 0.65 & 22.4 & 4.0 & 3.0 & 2 \\
\hline 107 & I17599-2148 & 18:03:01.83 & $-21: 48: 09.0$ & 18.6 & 2.99 & 5.4 & 1.15 & 32.0 & 5.2 & 3.4 & 1,2 \\
\hline 108 & I1 $8032-2032$ & 18:06:14.99 & $-20: 31: 35.4$ & 4.3 & 5.15 & 3.4 & 1.27 & 32.1 & 5.4 & 3.5 & 2 \\
\hline 109 & I18056-1952 & $18: 08: 38.18$ & $-19: 51: 49.0$ & 66.7 & 8.55 & 1.6 & 2.32 & 25.1 & 5.7 & 4.4 & 2 \\
\hline 110 & I18075-2040 & $18: 10: 34.82$ & $-20: 39: 16.1$ & 31.5 & 3.08 & 5.3 & 0.27 & 23.0 & 3.0 & 2.2 & 2 \\
\hline 111 & I18079-1756 & $18: 10: 50.60$ & $-17: 55: 47.2$ & 18 & 1.83 & 6.6 & 0.44 & 25.6 & 3.9 & 2.6 & 2 \\
\hline 112 & I18089-1732 & $18: 11: 51.28$ & $-17: 31: 33.4$ & 33.5 & 2.5 & 5.9 & 0.90 & 23.4 & 4.3 & 3.1 & 2 \\
\hline 113 & I18110-1854 & $18: 14: 00.83$ & $-18: 53: 28.2$ & 37 & 3.37 & 5.1 & 0.87 & 28.9 & 4.8 & 3.2 & 2 \\
\hline 114 & I18116-1646 & $18: 14: 35.76$ & $-16: 45: 40.8$ & 48.5 & 3.94 & 4.6 & 0.99 & 33.8 & 5.1 & 3.1 & 2 \\
\hline 115 & I18117-1753 & $18: 14: 39.14$ & $-17: 52: 01.3$ & 36.7 & 2.57 & 5.9 & 1.02 & 23.6 & 4.6 & 3.5 & 2 \\
\hline 116 & I18134-1942 & $18: 16: 22.12$ & $-19: 41: 27.0$ & 10.6 & 1.25 & 7.1 & 0.23 & 20.7 & 3.0 & 2.4 & 2 \\
\hline 117 & I18139-1842 & $18: 16: 51.72$ & $-18: 41: 35.2$ & 39.8 & 3.02 & 5.4 & 0.45 & 40.4 & 4.9 & 2.5 & 2 \\
\hline 118 & I18159-1648 & $18: 18: 54.34$ & $-16: 47: 51.9$ & 22 & 1.48 & 6.9 & 0.48 & 21.6 & 3.8 & 2.8 & 2 \\
\hline 119 & I18182-1433 & $18: 21: 09.22$ & $-14: 31: 46.8$ & 59.1 & 4.71 & 4.1 & 0.71 & 24.7 & 4.3 & 3.1 & 2 \\
\hline 120 & I1 $8223-1243$ & $18: 25: 10.58$ & $-12: 42: 24.2$ & 44.8 & 3.37 & 5.3 & 0.83 & 23.7 & 4.2 & 2.9 & 2 \\
\hline 121 & I18228-1312 & $18: 25: 41.81$ & $-13: 10: 23.3$ & 32.3 & 3.21 & 5.4 & 0.81 & 29.8 & 4.7 & 3.0 & 2 \\
\hline 122 & I18236-1205 & $18: 26: 25.65$ & $-12: 04: 01.6$ & 25.9 & 2.17 & 6.3 & 0.62 & 19.5 & 3.5 & 2.9 & 2 \\
\hline
\end{tabular}


Table A1 - continued Basic parameters of the targets in the ATOMS survey.

\begin{tabular}{|c|c|c|c|c|c|c|c|c|c|c|c|}
\hline Number & IRAS & RA & DEC & $\begin{array}{c}V_{\mathrm{lsr}} \\
\left(\mathrm{km} \mathrm{s}^{-1}\right)\end{array}$ & $\begin{array}{c}\text { Distance } \\
(\mathrm{kpc})\end{array}$ & $\begin{array}{l}R_{\mathrm{GC}} \\
(\mathrm{kpc})\end{array}$ & $\begin{array}{c}\text { Radius } \\
\text { (pc) }\end{array}$ & $\begin{array}{c}T_{\text {dust }} \\
(\mathrm{K})\end{array}$ & $\begin{array}{c}\log \left(L_{\text {bol }}\right) \\
\left(\mathrm{L}_{\odot}\right)\end{array}$ & $\begin{array}{c}\log \left(M_{\text {clump }}\right) \\
\left(\mathrm{M}_{\odot}\right)\end{array}$ & refs \\
\hline 123 & I18264-1152 & $18: 29: 14.28$ & $-11: 50: 27.0$ & 43.2 & 3.33 & 5.3 & 0.81 & 20.3 & 3.9 & 3.2 & 2 \\
\hline 124 & I18290-0924 & $18: 31: 43.23$ & $-09: 22: 28.5$ & 84.2 & 5.34 & 4.0 & 1.09 & 22.1 & 4.0 & 3.2 & 2 \\
\hline 125 & I18308-0503 & $18: 33: 29.42$ & $-05: 00: 55.1$ & 42.9 & 3.1 & 5.7 & 0.59 & 31.0 & 4.3 & 2.6 & 1 \\
\hline 126 & I18311-0809 & $18: 33: 53.43$ & $-08: 07: 14.4$ & 113 & 6.06 & 3.7 & 2.12 & 25.4 & 5.0 & 3.7 & 2 \\
\hline 127 & I18314-0720 & $18: 34: 10.25$ & $-07: 18: 00.1$ & 101.5 & 5.82 & 3.9 & 2.54 & 30.4 & 5.7 & 3.8 & 2 \\
\hline 128 & I18316-0602 & $18: 34: 20.58$ & $-05: 59: 41.6$ & 42.8 & 2.09 & 6.5 & 0.56 & 23.4 & 4.0 & 2.9 & 2 \\
\hline 129 & I18317-0513 & $18: 34: 25.50$ & $-05: 10: 53.5$ & 42.1 & 2.18 & 6.5 & 0.32 & 31.0 & 4.8 & 3.2 & 1,2 \\
\hline 130 & I18317-0757 & $18: 34: 24.90$ & $-07: 54: 50.6$ & 80.7 & 4.79 & 4.4 & 1.93 & 30.4 & 5.2 & 3.4 & 2 \\
\hline 131 & I18341-0727 & $18: 36: 49.82$ & $-07: 24: 53.0$ & 112.7 & 6.04 & 3.8 & 2.02 & 25.7 & 5.1 & 3.7 & 2 \\
\hline 132 & I18411-0338 & $18: 43: 46.07$ & $-03: 35: 33.0$ & 102.8 & 7.41 & 4.0 & 1.98 & 27.6 & 5.1 & 3.7 & 2 \\
\hline 133 & I18434-0242 & $18: 46: 03.51$ & $-02: 39: 26.7$ & 97.2 & 5.16 & 4.7 & 1.48 & 35.5 & 5.7 & 3.6 & 2 \\
\hline 134 & I18440-0148 & $18: 46: 36.22$ & $-01: 45: 23.7$ & 97.5 & 5.16 & 4.7 & 0.43 & 33.0 & 4.1 & 2.3 & 2 \\
\hline 135 & I18445-0222 & $18: 47: 09.76$ & $-02: 18: 47.6$ & 86.9 & 5.16 & 4.7 & 1.30 & 27.0 & 4.6 & 3.4 & 2 \\
\hline 136 & I18461-0113 & $18: 48: 41.83$ & $-01: 10: 01.4$ & 96.1 & 5.16 & 4.7 & 0.65 & 27.0 & 4.4 & 3.1 & 2 \\
\hline 137 & I18469-0132 & $18: 49: 33.15$ & $-01: 29: 04.2$ & 87 & 5.16 & 4.7 & 0.68 & 32.2 & 4.8 & 3.0 & 2 \\
\hline 138 & I18479-0005 & $18: 50: 30.79$ & $-00: 01: 58.2$ & 14.6 & 12.96 & 7.5 & 2.45 & 34.2 & 6.1 & 4.2 & 2 \\
\hline 139 & $\mathrm{I} 18502+0051$ & $18: 55: 22.63$ & $00: 59: 16.0$ & 53 & 7.1 & 4.7 & 1.24 & 30.0 & 5.3 & 3.9 & 1 \\
\hline 140 & $\mathrm{I} 18507+0110$ & $18: 53: 18.42$ & 01:15:00.1 & 57.2 & 1.56 & 7.1 & 0.44 & 29.2 & 4.8 & 3.2 & 2 \\
\hline 141 & $\mathrm{I} 18507+0121$ & 18:53:18.12 & $01: 25: 22.7$ & 57.9 & 1.56 & 7.1 & 0.29 & 22.7 & 3.5 & 2.6 & 2 \\
\hline 142 & $\mathrm{I} 18517+0437$ & $18: 54: 14.13$ & $04: 41: 46.2$ & 43.9 & 2.36 & 6.6 & 0.23 & 32.0 & 4.6 & 2.9 & 1,2 \\
\hline 143 & $\mathrm{I} 18530+0215$ & $18: 55: 33.61$ & 02:19:09.0 & 74.1 & 4.67 & 5.3 & 1.06 & 26.1 & 4.7 & 3.3 & 2 \\
\hline 144 & I19078+0901 & 19:10:13.41 & 09:06:10.4 & 2.9 & 11.11 & 7.6 & 4.26 & 33.3 & 6.9 & 5.0 & 2 \\
\hline 145 & I19095+0930 & 19:11:53.90 & $09: 35: 45.9$ & 43.7 & 6.02 & 5.8 & 0.64 & 34.9 & 5.1 & 3.1 & 2 \\
\hline 146 & I19097+0847 & 19:12:09.09 & $08: 52: 10.8$ & 58 & 8.47 & 6.2 & 2.01 & 23.3 & 5.0 & 3.8 & 2 \\
\hline
\end{tabular}

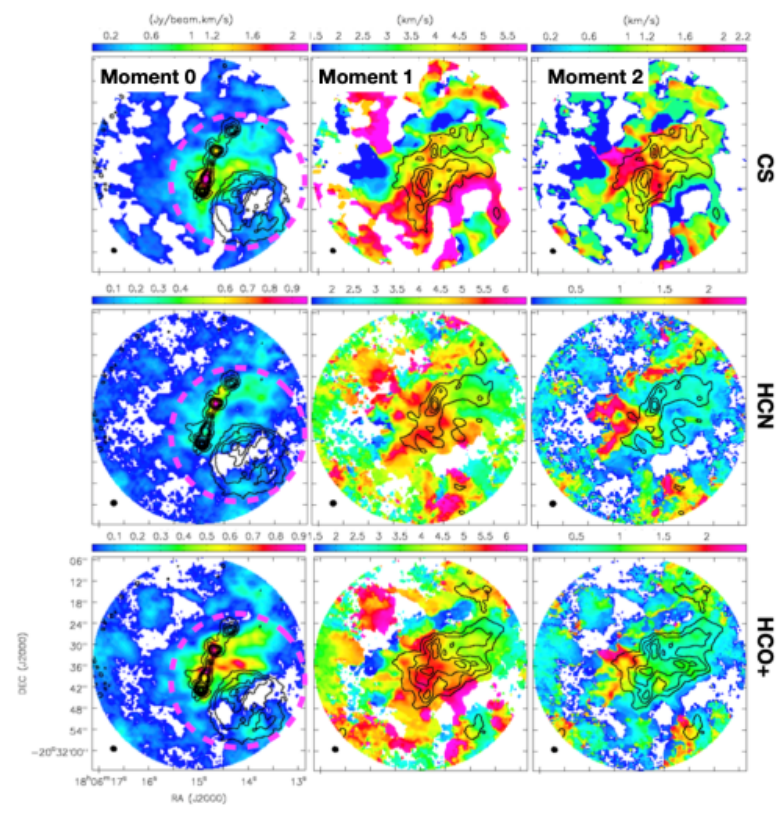

Figure A1. The left, middle and right columns present the integrated intensity (Moment 0) maps, intensity-weighted velocity maps (Moment 1) and intensityweighted velocity dispersion (Moment 2) maps of G9.62+0.19 for the gas tracers indicated on the right of each row. The names of tracers are labeled to the right. The contours on Moment 0 maps show the $3 \mathrm{~mm}$ continuum emission. The contour levels are $[0.01,0.03,0.05,0.07,0.1,0.2,0.4,0.6,0.8] \times 74.1 \mathrm{mJy}$ beam ${ }^{-1}$. The contours on Moment 1 and Moment 2 maps show the corresponding integrated intensity maps of various gas tracers. The contours are [0.2,0.4,0.6,0.8] $\times \mathrm{F}_{\text {peak }}$. The peak integrated intensities $\left(F_{\text {peak }}\right)$ for $\mathrm{CS}, \mathrm{HCN}, \mathrm{HCO}^{+}$are $2.16,0.97$, and $0.93 \mathrm{Jy} \mathrm{beam}^{-1} \mathrm{~km} \mathrm{~s}^{-1}$, respectively. The magenta dashed circle on Moment 0 map shows the clump boundary traced by $\mathrm{H}^{13} \mathrm{CN}$. 


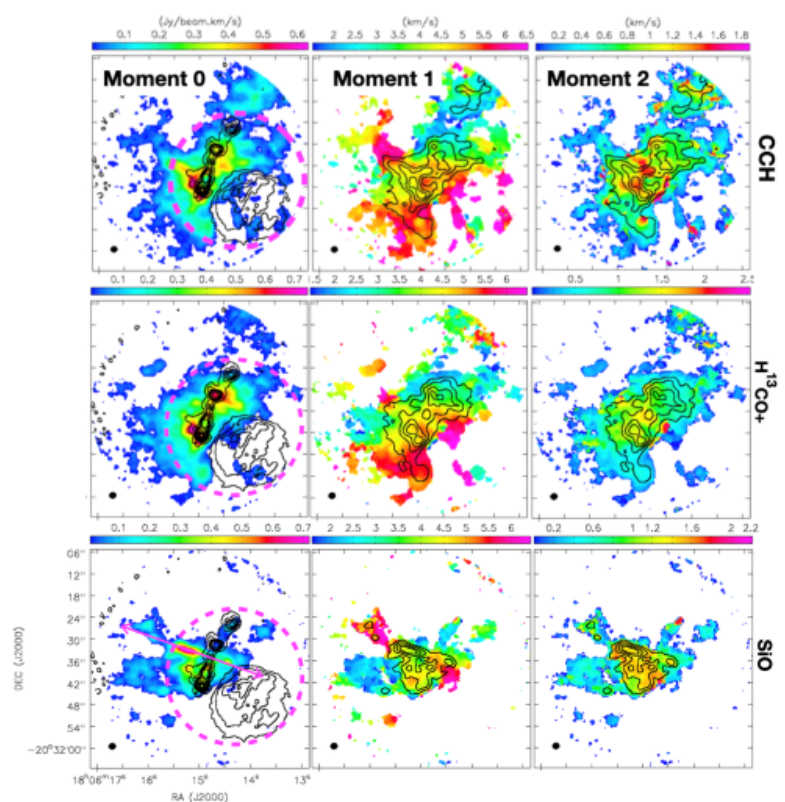

Figure A2. The images and contours are same as in Figure A1. The peak integrated intensities $\left(F_{\text {peak }}\right)$ for $\mathrm{CCH}, \mathrm{H}^{13} \mathrm{CO}^{+}$and $\mathrm{SiO}$ are $0.63,0.75$, and 0.72 Jy beam ${ }^{-1} \mathrm{~km} \mathrm{~s}^{-1}$, respectively. The magenta dashed circle on the Moment 0 maps show the clump boundary traced by $\mathrm{H}^{13} \mathrm{CN}$. The magenta arrow represents the direction of the bipolar outflow driven by MM6.

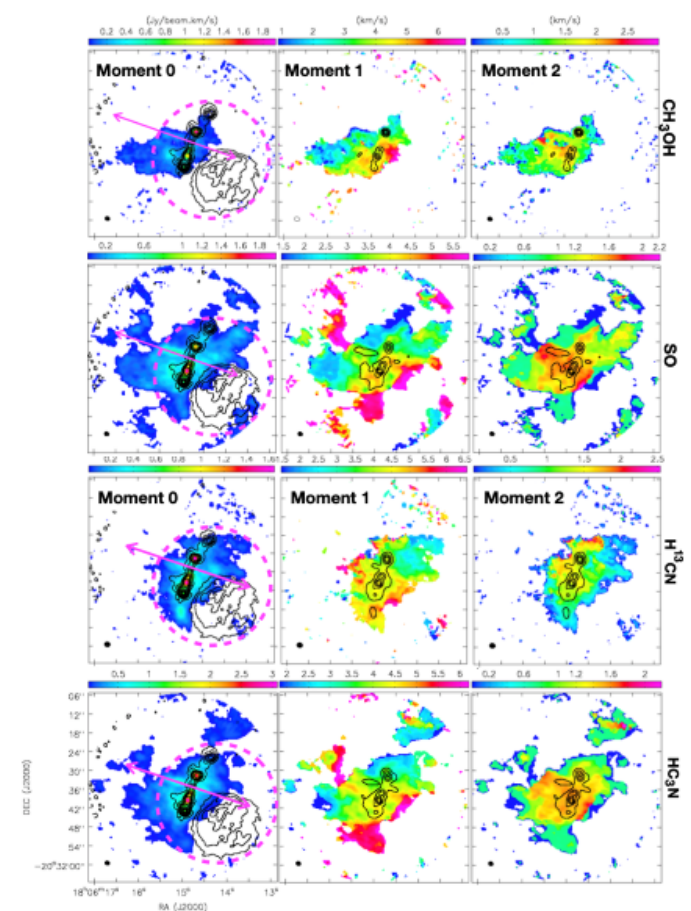

Figure A3. The images and contours are same as in Figure A1. The peak integrated intensities $\left(F_{\text {peak }}\right)$ for $\mathrm{CH}_{3} \mathrm{OH}, \mathrm{SO}, \mathrm{H}^{13} \mathrm{CN}$ and $\mathrm{HC}_{3} \mathrm{~N}$ are $1.94,1.98,1.65$, and $3.09 \mathrm{Jy}_{\text {beam }}{ }^{-1} \mathrm{~km} \mathrm{~s}^{-1}$, respectively. The magenta dashed circle on the Moment 0 maps show the clump boundary traced by $\mathrm{H}^{13} \mathrm{CN}$. The magenta arrow represents the direction of the bipolar outflow driven by MM6. 
Author affiliations:

${ }^{1}$ Key Laboratory for Research in Galaxies and Cosmology, Shanghai Astronomical Observatory, Chinese Academy of Sciences, 80 Nandan Road, Shanghai 200030, PeopleâĂŹs Republic of China

${ }^{2}$ Korea Astronomy and Space Science Institute, 776 Daedeokdaero, Yuseong-gu, Daejeon 34055, Republic of Korea

${ }^{3}$ Department of Astronomy, The University of Texas at Austin, 2515 Speedway, Stop C1400, Austin, TX 78712-1205, USA

${ }^{4}$ University of Science and Technology, Korea (UST), 217 Gajeong-ro, Yuseong-gu, Daejeon 34113, Republic of Korea

${ }^{5}$ Jet Propulsion Laboratory, California Institute of Technology, 4800 Oak Grove Drive, Pasadena, CA 91109, USA

${ }^{6}$ Institute of Astronomy and Astrophysics, Academia Sinica. 11F of Astronomy-Mathematics Building, AS/NTU No. 1, Section 4, Roosevelt Rd., Taipei 10617, Taiwan

${ }^{7}$ Center for Astrophysics | Harvard \& Smithsonian, 60 Garden Street, Cambridge, MA 02138, USA

${ }^{8}$ National Astronomical Observatory of Japan, National Institutes of Natural Sciences, 2-21-1 Osawa, Mitaka, Tokyo 181-8588, Japan

${ }^{9}$ Kavli Institute for Astronomy and Astrophysics, Peking University, 5 Yiheyuan Road, Haidian District, Beijing 100871, People's Republic of China

${ }^{10}$ Department of Physics, P.O. Box 64, FI-00014, University of Helsinki, Finland

${ }^{11}$ Departamento de Astronomía, Universidad de Chile, Las Condes, Santiago, Chile

${ }^{12}$ School of Physics, University of New South Wales, Sydney, NSW 2052, Australia

${ }^{13}$ School of Space Research, Kyung Hee University, Yongin-Si, Gyeonggi-Do 17104, Republic of Korea

${ }^{14}$ National Astronomical Observatories, Chinese Academy of Sciences, Beijing 100101, China

${ }^{15}$ University of Chinese Academy of Sciences, Beijing 100049, China

${ }^{16}$ Astronomy Department, University of California, Berkeley, CA 94720, USA

${ }^{17}$ Department of Astronomy, Yunnan University, and Key Laboratory of Astroparticle Physics of Yunnan Province, Kunming, 650091, People's Republic of China

${ }^{18}$ IRAP, Université de Toulouse, CNRS, UPS, CNES, Toulouse, France

${ }^{19}$ Indian Institute of Space Science and Technology, Thiruvananthapuram 695 547, Kerala, India

${ }^{20}$ Eötvös Loránd University, Department of Astronomy, Pázmány Péter sétány 1/A, H-1117, Budapest, Hungary

${ }^{21}$ Department of Astronomy, Peking University, 100871, Beijing, People's Republic of China

${ }^{22}$ Departamento de Astronomía, Universidad de Concepción, Av. Esteban Iturra s/n, Distrito Universitario, 160-C, Chile

${ }^{23}$ College of Science, Yunnan Agricultural University, Kunming, 650201, People's Republic of China

${ }^{24}$ Shanghai Astronomical Observatory, Chinese Academy of Sciences, 80 Nandan Road, Shanghai 200030 People's Republic of China

${ }^{25}$ Key Laboratory of Radio Astronomy, Chinese Academy of Sciences, Nanjing 210008, People's Republic of China

${ }^{26}$ School of Physics and Astronomy, Sun Yat-sen University, 2 Daxue Road, Zhuhai, Guangdong, 519082, People's Republic of China

${ }^{27}$ NAOC-UKZN Computational Astrophysics Centre, University of KwaZulu-Natal, Durban 4000, South Africa

${ }^{28}$ SOFIA Science Centre, USRA, NASA Ames Research Centre, MS-12, N232, Moffett Field, CA 94035, USA

This paper has been typeset from a $\mathrm{T}_{\mathrm{E}} \mathrm{X} / \mathrm{LT}_{\mathrm{E}} \mathrm{X}$ file prepared by the author. 